\title{
The omega deformation, branes, integrability and Liouville theory
}

\author{
Nikita Nekrasov ${ }^{a, b}$ and Edward Witten ${ }^{c}$ \\ ${ }^{a}$ Institut des Hautes Études Scientifiques, \\ Le Bois-Marie 35, route de Chartres, 91440 Bures-sur-Yvette France \\ ${ }^{b}$ Simons Center for Geometry and Physics, Stony Brook University, \\ Stony Brook, 11794 New York U.S.A. \\ ${ }^{c}$ School of Natural Sciences, Institute for Advanced Study, \\ Einstein Drive, Princeton, NJ 08540 U.S.A. \\ E-mail: nikitastring@gmail.com, witten@ias.edu
}

ABSTRACT: We reformulate the $\Omega$-deformation of four-dimensional gauge theory in a way that is valid away from fixed points of the associated group action. We use this reformulation together with the theory of coisotropic $A$-branes to explain recent results linking the $\Omega$-deformation to integrable Hamiltonian systems in one direction and Liouville theory of two-dimensional conformal field theory in another direction.

Keywords: Supersymmetric gauge theory, Integrable Field Theories, Brane Dynamics in Gauge Theories

ARXiv EPRINT: 1002.0888 


\section{Contents}

1 Introduction 1

2 Some background $\quad 3$

2.1 The basic link between gauge theory and integrability 3

2.1.1 Validity of the WKB approximation 5

$\begin{array}{ll}2.1 .2 & \text { Observables }\end{array}$

$\begin{array}{lll}2.2 & \text { Complex integrable systems and their real sections } & 7\end{array}$

2.3 Coisotropic $A$-branes 9

2.3.1 The Hyper-Kahler case 13

$\begin{array}{lll}2.3 .2 & \text { First among equals } & 14\end{array}$

$\begin{array}{ll}2.4 \text { A little differential geometry } & 16\end{array}$

$\begin{array}{lll}\text { 2.4.1 General Half-BPS condition } & 17\end{array}$

$\begin{array}{lll}2.4 .2 & \text { Role of generalized complex geometry } & 18\end{array}$

$\begin{array}{ll}2.4 .3 \text { "Rotation" group } & 19\end{array}$

3 Compactification and $\Omega$-deformation $\quad 20$

$\begin{array}{lll}3.1 & \text { The undeformed case } & 21\end{array}$

3.1.1 Support of the brane 23

3.1.2 Two-dimensional interpretation of the supersymmetries 25

3.1.3 A mixed $A B$-model 26

$\begin{array}{ll}3.1 .4 & \text { More on compactification }\end{array}$

$\begin{array}{lll}3.2 & \text { Rethinking the } \Omega \text {-deformation } & 28\end{array}$

$\begin{array}{ll}3.2 .1 & \text { The Standard formulation } \\ 3.2 .29\end{array}$

$\begin{array}{lll}3.2 .2 & \text { An alternative description } & 30\end{array}$

3.3 The deformed brane 33

3.3.1 The case $\varepsilon \rho \rightarrow \infty \quad 34$

$\begin{array}{ll}\text { 3.3.2 The general case } & 36\end{array}$

$\begin{array}{lll}3.3 .3 & \text { A canonical coisotropic brane } & 37\end{array}$

$\begin{array}{lll}3.4 & \text { Boundary conditions at the far end } & 39\end{array}$

3.4.1 Gauge theory realization $\quad 41$

3.4.2 Alternative compactification to two dimensions 43

$\begin{array}{lll}3.4 .3 & \text { Eigenvalues and opers } & 44\end{array}$

4 Conformal blocks from four dimensions $\quad 46$

4.1 Gauge theory and Liouville theory 46

$\begin{array}{lll}4.2 & \Omega \text {-Deformation } & 47\end{array}$

4.3 Two-dimensional description $\quad 48$

4.4 The Kahler parameter of the sigma model 49

4.5 More about the branes 51

$\begin{array}{lll}4.6 & \text { A practice case } & 52\end{array}$ 
4.7 The brane of opers 54

4.8 Physical states from the brane of opers 56

$\begin{array}{lll}4.9 & \text { Observables } & 58\end{array}$

4.9.1 Wilson and 't Hooft operators 60

4.9.2 Winding states of the $A$-model 61

4.10 Partition functions $\quad 62$

4.11 Flatness 63

$\begin{array}{ll}4.12 \text { Including surface operators } & 64\end{array}$

A The general $\Omega$-deformation in toroidal compactification $\quad 66$

$\begin{array}{lll}\text { A.1 The setup } & 66\end{array}$

$\begin{array}{lll}\text { A.2 The familiar story } & 67\end{array}$

$\begin{array}{ll}\text { A.2.1 The bosonic lagrangian } & 68\end{array}$

$\begin{array}{lll}\text { A.2.2 } & T \text {-duality to } \mathcal{M}_{H} & 68\end{array}$

$\begin{array}{lll}\text { A.3 The } \Omega \text {-deformation } & 70\end{array}$

A.3.1 The supersymmetry of the $\Omega$-deformed theory 72

$\begin{array}{lll}\text { A.3.2 Undoing the } \Omega \text {-deformation } & 72\end{array}$

$\begin{array}{lll}\text { A.3.3 Rotating the fields } & 73\end{array}$

$\begin{array}{lll}\text { A.3.4 T-duality, again } & 73\end{array}$

$\begin{array}{lll}\text { A.4 Rotated complex structures } & 74\end{array}$

A.4.1 Mapping to the $A$-Model $\quad 76$

$\begin{array}{lll}\text { A.5 Dehn twists } & 77\end{array}$

\section{Introduction}

In this paper, we will apply methods of two-dimensional sigma-models to two different problems in four-dimensional gauge theory.

The first problem involves the relation of gauge theory to quantum integrable systems. Vacua of massive two-dimensional gauge theories with $\mathcal{N}=2$ supersymmetry correspond unexpectedly $[1-3]$ to the quantum eigenstates of a quantum integrable system. This correspondence has recently been extended $[4,5]$ to a much wider class of examples. In the present paper, we will approach this rather surprising relation from a new angle. We focus on what is perhaps the most challenging example of the correspondence in question. This is the case [6] that the two-dimensional theory arises by reducing a four-dimensional $\mathcal{N}=2$ supersymmetric gauge theory to two dimensions with the help of the $\Omega$ deformation [7]. The resulting theories are associated to quantum integrable systems that arise by quantizing a finite-dimensional classical phase space. By contrast, many purely two-dimensional examples are more directly described in terms of quantum spin systems.

Our basic idea is to map this problem to a brane construction in two dimensions. Under certain conditions, a two-dimensional $A$-model admits unusual branes [10] whose existence brings noncommutativity into the $A$-model in several related ways [11-14]. Of most direct 
relevance to us is an $A$-brane construction that leads to quantization of finite-dimensional classical phase spaces [14]. In this construction, integrability is natural. The construction involves a pair of branes; one is an ordinary Lagrangian $A$-brane, and the second, which has been called the canonical coisotropic $A$-brane $\mathcal{B}_{\mathrm{cc}}$, is the most simple example of the unusual $A$-branes introduced in [10].

In section 2, we describe some background that may be helpful. We recall the basic reason that massive supersymmetric gauge theories in two dimensions are related to integrability. We review a variety of facts about two-dimensional sigma-models and their relation to four-dimensional gauge theories, including the brane construction [14] that will be our main tool.

In section 3 , we describe how to study the $\Omega$-deformation via this framework. Here, as in [6], we consider the $\Omega$-deformation of $\mathcal{N}=2$ super Yang-Mills theory on $\mathbb{R}^{4}$, defined by a $U(1)$ action that leaves fixed a two-plane $\mathbb{R}^{2} \subset \mathbb{R}^{4}$. There is an immediate problem, as it appears that both the $\Omega$-deformed Lagrangian and the supersymmetry preserved by the $\Omega$-deformation are not what we need to make contact with the brane construction of [14]. To overcome this difficulty, we give a new interpretation of the $\Omega$-deformation. The $\Omega$ deformation is defined using a vector field $V$ that generates a $\mathrm{U}(1)$ symmetry of spacetime. As originally defined in [7], building on $[1,8,9]$, the $\Omega$ deformation involves a deformation of the Lagrangian that preserves part of the supersymmetry. We will give an alternative description of the $\Omega$-deformation that is valid (for our purposes, which do not depend on the precise choice of a $\mathrm{U}(1)$-invariant metric) away from the zeroes of $V$ : by a change of field variables, one can remove the deformation from the Lagrangian while rotating the unbroken supersymmetry.

Taking this into account, we show that $\Omega$-deformed supersymmetric gauge theory, in the situation considered in [6], reduces naturally to an $A$-model in two dimensions, with precisely the brane setup of [14]. The most unusual part of this construction is the exotic $A$-brane $\mathcal{B}_{\text {cc }}$. It arises in giving a two-dimensional interpretation to what in four dimensions are simply the $\mathrm{U}(1)$ fixed points.

Section 4 is devoted to applying our framework to a very different-sounding problem. Here our aim is to make contact with remarkable results [15] linking the $\Omega$-deformation in four dimensions with Liouville theory (and its higher rank analogs) in two dimensions. Our method can be applied to this situation in an interesting way and answers some of the questions, although many points are not yet clear. In this application, the important branes are all rotated or dual versions of the coisotropic brane $\mathcal{B}_{\mathrm{cc}}$.

Though our discussion is applicable to any $\mathcal{N}=2$ supersymmetric gauge theory, we often specialize to a convenient and large class of such theories which arise by compactification of the six-dimensional $(0,2)$ model of type $G$ (here $G$ is a simple and simply-laced Lie group) on a Riemann surface $C$. For every choice of a system of $A$-cycles on $C$, one gets in four dimensions a gauge theory realization [16] in which the gauge group is a product of copies of $G$. We call these generalized quiver theories. Compactification on a two-torus reduces a generalized quiver theory to a sigma-model in two dimensions in which the target space is $\mathcal{M}_{H}$, the moduli space of Higgs bundles on $C$, endowed with a hyper-Kahler metric [17]. 
To keep things simple, a number of calculations in the body of this paper are carried out only for compactification on a rectangular two-torus and only for special values of the parameters of the $\Omega$-deformation. A more complete treatment is given in the appendix.

Our strategy throughout this paper is qualitatively similar to many applications of toric geometry in string theory. For example, see [18]. Also, the brane construction we use in section 3 to obtain an eigenvalue problem for the commuting Hamiltonians of a quantum integrable system is a cousin of a construction that has been analyzed in the literature on geometric Langlands [19-22]. Our (limited) understanding of the relation is described in section 3.4.3. Finally, our results in section 4 are qualitatively in agreement with previous arguments suggesting a relation between duality of Liouville theory and what is often called quantum geometric Langlands [23-26]. We hope it will prove possible to make this connection more precise.

\section{Some background}

The present section is devoted to describing some background that may be helpful. None of these results are new.

In section 2.1, we will review the basic reason that there is a link between twodimensional massive supersymmetric gauge theories and integrability. In section 2.2, we discuss some generalities about what it might mean to quantize a complex integrable system. In section 2.3 , we review some relevant facts about the two-dimensional topological $A$-model and recall how $A$-branes can be used for quantization. In section 2.4, we describe some pertinent differential geometry.

\subsection{The basic link between gauge theory and integrability}

For illustrative purposes, we consider a two-dimensional $\mathcal{N}=2$ supersymmetric gauge theory with $(2,2)$ supersymmetry and a gauge group $\mathrm{U}(1)^{r}$. (The general case of a gauge group $G$ of rank $r$ can be treated similarly; it leads to a nonabelian but equally tractable version of the Lagrangian (2.2).)

The $\mathrm{U}(1)^{r}$ gauge theory has $r$ vector multiplets. Their gauge-invariant content can be described by the twisted chiral multiplets

$$
\Sigma_{a}=\sigma_{a}-i \sqrt{2} \theta^{+} \bar{\lambda}_{a+}-i \sqrt{2} \bar{\theta}^{-} \lambda_{a-}+\sqrt{2} \theta^{+} \bar{\theta}^{-}\left(D_{a}-i \star F_{a}\right)+\ldots, a=1, \ldots, r
$$

where $\theta^{ \pm}$and $\bar{\theta}^{ \pm}$are superspace coordinates, $\sigma_{a}$ and $\lambda_{a}$ are ordinary scalar and fermi fields, $D_{a}$ are auxiliary fields, $F_{a}$ is the field strength of the $a^{t h} \mathrm{U}(1)$ gauge field, and $\star$ is the Hodge star operator. We are interested in theories in which all vacua are massive, that is, admit only excitations with positive mass. For this to be the case, any chiral multiplets that are present in the theory must be massive; they can be integrated out, possibly making contributions to the effective twisted chiral superpotential $\widetilde{W}\left(\Sigma_{1}, \ldots, \Sigma_{r}\right)$ of the vector multiplets. The theory is then massive if $\widetilde{W}$ is sufficiently generic.

If so, the only important part of the effective action at low energies is the contribution of $\widetilde{W}$ :

$$
I_{\widetilde{W}}=\frac{1}{2 \sqrt{2}} \int d^{2} x\left(\mathrm{~d} \theta^{+} \mathrm{d} \bar{\theta}^{-} \widetilde{W}\left(\Sigma_{1}, \ldots, \Sigma_{r}\right)+\text { c.c. }\right) .
$$


This is the action of a topological field theory; by dropping higher derivative terms in the action, we have effectively taken the masses of all excitations to infinity. The quantum states obtained by quantizing (2.2) are the same as the vacua in the infinite volume limit of an underlying physical theory whose action consists of $I_{\widetilde{W}}$ plus irrelevant terms of higher dimension.

After performing the $\theta$ integrals, $I_{\widetilde{W}}$ gives for the fermions $\lambda_{a}$ a mass matrix $m_{a b}=$ $\partial^{2} \widetilde{W} / \partial \sigma^{a} \partial \sigma^{b}$. If this is nondegenerate (invertible) in every vacuum, as we will assume, then the fermions are "massive" (but nonpropagating in the approximation of (2.2), as they have no kinetic energy). Let us look more closely at the bosonic part of $I_{\widetilde{W}}$, which turns out to be

$$
I_{\widetilde{W}, B}=\int \mathrm{d}^{2} x \sum_{a}\left(D_{a} \operatorname{Re} \frac{\partial \widetilde{W}}{\partial \sigma_{a}}+\star F_{a} \operatorname{Im} \frac{\partial \widetilde{W}}{\partial \sigma_{a}}\right) .
$$

Since the part of $I_{\widetilde{W}, B}$ that involves the auxiliary fields $D_{a}$ has no derivatives, the effect of the terms involving $D_{a}$ is simply to impose constraints:

$$
\operatorname{Re} \frac{\partial \widetilde{W}}{\partial \sigma_{a}}=0
$$

Let $\boldsymbol{P}$ be the locus (in a copy of $\mathbb{C}^{r}$ parametrized by the $\sigma_{a}$ ) defined by these constraints. If $\widetilde{W}$ is sufficiently generic, then $p_{a}=\operatorname{Im} \partial \widetilde{W} / \partial \sigma_{a}$ is a good system of coordinates on $\boldsymbol{P}$.

We will analyze the dynamics of the fields $p_{a}$ and the $\mathrm{U}(1)$ gauge fields $A_{a}$ on a twomanifold $\mathbb{R} \times S^{1}$, with metric $\mathrm{d} s^{2}=\mathrm{d} t^{2}-\mathrm{d} x^{2}$, where $t$ is real-valued and $x \cong x+R$ (for some constant $R$ ) parametrizes $S^{1}$. We work in the gauge in which the $t$ components of all gauge fields vanish, that is $A_{t, a}=0$. The Gauss law constraint $\delta I_{\widetilde{W}, B} / \delta A_{t, a}=0$ is $\partial p_{a} / \partial \sigma=0$, so in this gauge, the $p_{a}$ are functions of $t$ only. We can further fix the gauge so that the spatial parts of the gauge fields are constants: $A_{x, a}=\phi_{a} / R$, where the $\phi_{a}$ are angular variables that depend only on $t$. The action then reduces to

$$
I^{\prime}=\int \mathrm{d} t \sum_{a} p_{a} \frac{\mathrm{d} \phi_{a}}{\mathrm{~d} t}
$$

This is an integrable system written in action-angle coordinates. The $p_{a}$ are the action variables and the $\phi_{a}$ are the angle variables. The nonzero Poisson brackets are $\left\{p_{a}, \phi_{b}\right\}=$ $\delta_{a b}$. In particular, the $p_{a}$ are Poisson-commuting and quantum states can be labeled by the values of the $p_{a}$.

However, not all values of the $p_{a}$ occur. The reason for this is that the space $T$ obtained by specifying the values of the $p_{a}$ is compact; it is a torus, parametrized by the $\phi_{a}$. In the WKB approximation, the good values of the $p_{a}$ are those for which the one-form $\omega=\sum_{a} p_{a} \mathrm{~d} \phi_{a}$ has all its periods integer multiples of $2 \pi$. Each $\phi_{a}$ parametrizes a circle $S_{a}$, and the basic periods are $\int_{S_{a}} \mathrm{~d} \phi_{b}=2 \pi \delta_{a b}$. So the condition for $\omega$ to have all integer periods is simply that the $p_{a}$ should be integers. Recalling the definition of the $p_{a}$, our conclusion is that

$$
\operatorname{Im} \frac{\partial \widetilde{W}}{\partial \sigma_{a}}=n_{a}, n_{a} \in \mathbb{Z}
$$


Evidently, this result can be combined with eq. (2.4) to give the holomorphic relation

$$
\frac{\partial \widetilde{W}}{\partial \sigma_{a}}=i n_{a}, n_{a} \in \mathbb{Z}
$$

or equivalently

$$
\exp \left(2 \pi \frac{\partial \widetilde{W}}{\partial \sigma_{a}}\right)=1
$$

which characterizes the quantum states of the topological field theory with action $I_{\widetilde{W}}$, or equivalently the vacua of an underlying massive theory whose action differs from $I_{\widetilde{W}}$ by irrelevant operators.

Apart from possibly orienting the reader to the results of [1-6], the reason that we have explained these matters here is to draw a lesson that will be important for our derivation in the rest of this paper. The constraint (2.8) that determines the vacuum states is holomorphic in $\widetilde{W}$, but in the derivation the real and imaginary parts of $\partial \widetilde{W} / \partial \sigma_{a}$ have gone their separate ways. Since this happens just in the two-dimensional derivation, it will hopefully come as no surprise when something similar happens in deriving this story from four dimensions.

\subsubsection{Validity of the WKB approximation}

There remain two points to clarify about this derivation. First, we have presented (2.7) or (2.8) as the result of a WKB approximation, but actually in the context of supersymmetric gauge theory, these formulas are exact (modulo additive constants that will be discussed). This is a standard result; for example, see [27] for a derivation via path integrals. The basic idea of the derivation is to integrate over the gauge fields with the action (2.3). In the integral

$$
\int D A_{a} \exp \left(i \sum_{a} \int F_{a} \operatorname{Im} \partial \widetilde{W} / \partial \sigma_{a}\right)
$$

one would like to change variables from $A_{a}$ to $F_{a}$, using the fact that $F_{a}$ contains almost the full gauge-invariant content of $A_{a}$ and that (as the gauge group is abelian) the volume of the space of gauge fields with given curvature $F_{a}$ is independent of $F_{a}$. If the $F_{a}$ could be treated as arbitrary two-forms, the resulting integral

$$
\int D F_{a} \exp \left(i \sum_{a} \int F_{a} \operatorname{Im} \partial \widetilde{W} / \partial \sigma_{a}\right)
$$

would simply give a delta function setting $\operatorname{Im} \partial \widetilde{W} / \partial \sigma_{a}=0$. Instead, $F_{a}$ is constrained by Dirac quantization, $\int F_{a} / 2 \pi \in \mathbb{Z}$, which one can incorporate by including in the path integral a factor $\sum_{n_{a} \in \mathbb{Z}} \exp \left(-i \sum_{a} n_{a} \int F_{a}\right)$. With this factor included, the integral over the $F_{a}$ now gives a constraint $\operatorname{Im} \partial \widetilde{W} / \partial \sigma_{a}=n_{a}$.

The result $\partial \widetilde{W} / \partial \sigma_{a}=i n_{a}$ has a more elementary analog for chiral multiplets. If $\Phi_{i}=\phi_{i}+\theta \psi_{i}+\theta^{2} F_{i}+\ldots$ are chiral multiplets with superpotential $W\left(\Phi_{1}, \ldots, \Phi_{s}\right)$, then 
a vacuum is characterized by $\partial W\left(\phi_{1}, \ldots, \phi_{s}\right) / \partial \phi_{i}=0$. No integer analogous to $n_{a}$ enters because the auxiliary fields $F_{i}$ of the chiral multiplet are independent complex fields. The result (2.7) for twisted chiral multiplets of gauge theory is slightly more complicated because the "auxiliary field" $\widetilde{F}_{a}=D_{a}-i \star F_{a}$ in (2.1) is not an arbitrary complex field; its imaginary part is subject to Dirac quantization.

In general, it would be unrealistic to expect the WKB approximation to be always exact for an abstract integrable system written in action-angle variables. Indeed, if $p_{a}, \phi_{a}$ are a set of action-angle variables, then via a canonical transformation, one can map them (in many different ways) to another set of equally good action-angle variables $p_{a}^{\prime}, \phi_{a}^{\prime}$. It is impossible for the WKB approximation to be valid in every set of action-angle variables, so in general at best it will be exact only for a system of action-angle variables that is in some way distinguished. However, in the present context, the angle variables $\phi_{a}$ are indeed distinguished, as they originate from gauge fields; hence the conjugate variables $p_{a}$ are also distinguished, modulo possible additive constants which will indeed play a role. This makes it possible for the WKB approximation to be exact.

The constraint (2.4) is not holomorphic in $\widetilde{W}$, but the condition defining the vacuum states of the underlying gauge theory must be holomorphic. The WKB formula (2.6) has indeed combined with the constraint (2.4) to give the desired holomorphy in (2.8). A quantum correction to (2.6), apart from the possibility of adding a constant to the right hand side, would spoil holomorphy. By adding a constant, we mean replacing (2.6) with

$$
\operatorname{Im} \frac{\partial \widetilde{W}}{\partial \sigma_{a}}=n_{a}-\frac{\theta_{a}}{2 \pi}
$$

for some angles $\theta_{a}$. The $\theta_{a}$ must be constants, since if they were nontrivial functions of the $p_{a}$, this would spoil holomorphy.

For a perhaps fuller explanation, let us consider adding $\theta$-angles to the underlying gauge theory action:

$$
I_{\widetilde{W}} \rightarrow I_{\widetilde{W}}+\sum_{a} \theta_{a} \int \frac{F_{a}}{2 \pi}
$$

In the above derivation, the one-form $\omega=\sum_{a} p_{a} \mathrm{~d} \phi_{a}$ is then replaced by $\omega^{\prime}=\sum_{a}\left(p_{a}+\right.$ $\left.\theta_{a} / 2 \pi\right) \mathrm{d} \phi_{a}$, and this leads exactly to the generalized WKB condition (2.11). So this generalization is indeed something that we have to consider.

On the other hand, two-dimensional gauge theory is in general invariant under $\theta_{a} \rightarrow$ $\theta_{a}+2 \pi m_{a}$. (This is usually deduced from Dirac quantization of $\int F_{a}$, or alternatively proved by observing that the unitary operator $\exp \left(i \sum_{a} m_{a} \oint A_{a}\right)$ brings about a shift $\theta_{a} \rightarrow \theta_{a}+2 \pi m_{a}$.) Allowing for arbitrary integers $n_{a}$ in (2.11) insures that the spectrum has this invariance.

This discussion may raise the following question: is there a renormalization of the angles $\theta_{a}$ in going from the underlying Lagrangian to the exact condition (2.11) that characterizes the quantum states? In fact, this question is equivalent to asking whether the effective superpotential $\widetilde{W}$ has been identified correctly, since (as one can see by comparing eqs. (2.3) and (2.12)) introducing the angles $\theta_{a}$ is equivalent to changing $\widetilde{W}$ to 
$\widetilde{W}+i \sum_{a} \theta_{a} \sigma_{a} / 2 \pi$. So, given a microscopic theory, the problem of finding the right constants in the WKB formula is part of the problem of correctly computing the effective twisted chiral superpotential $\widetilde{W}$.

\subsubsection{Observables}

What are the natural observables of this integrable system? Going back to the underlying gauge theory description, the natural operators are those of the twisted chiral ring. The twisted chiral ring of this theory is a polynomial ring generated by the operators $\sigma_{a}$, $a=1, \ldots, r$. They commute, and the existence of these commuting operators can be regarded as an explanation of why a detailed analysis of this system has led to an integrable description in action-angle variables.

The derivation sketched above has led to a basis of quantum states, characterized by (2.8), in which the $\sigma_{a}$ have definite values. The chiral ring generators - and therefore all the operators of the chiral ring - are diagonal in this basis.

This was indeed the starting point of [1-6], where the conditions (2.8) that determine the eigenvalues of the chiral ring generators were interpreted in terms of Bethe ansatz equations of an integrable system presented not in action-angle variables but in some alternative and physically interesting description. The relation between the two pictures is not well understood, and we will not shed light on it in the present paper. Rather, in this paper, our goal is to understand the analog of the above two-dimensional derivation in the context of $\Omega$-deformed theories in four dimensions.

\subsection{Complex integrable systems and their real sections}

We want to put this in the context of $\mathcal{N}=2$ supersymmetric gauge theories in four dimensions. Let us denote as $\boldsymbol{B}$ the Coulomb branch of the moduli space of vacua of such a theory. (Depending on the spectrum of hypermultiplets, there may also be Higgs branches of vacua or mixed branches, but they will not be important in the present paper.) $\boldsymbol{B}$ parameterizes a family of abelian varieties. We denote as $X$ the total space of this fibration. $X$ is a complex symplectic manifold, with a holomorphic symplectic form $\Omega$. The fibers of the fibration $X \rightarrow \boldsymbol{B}$ are holomorphic submanifolds that are Lagrangian with respect to $\Omega$. This means that $X$ is a completely integrable Hamiltonian system in the complex sense [31]. The holomorphic functions on $\boldsymbol{B}$ are the action variables, and the fiber coordinates are the angle variables.

An important class of examples [16] is derived by compactification of the sixdimensional $(0,2)$ theory of type $G$ on a Riemann surface $C$. In this case, $X$ is a moduli space of Higgs bundles on $C$, with structure group $G$, and the fibration $X \rightarrow B$ is the Hitchin fibration. The integrable system is that of Hitchin [17] and we denote $X$ as $\mathcal{M}_{H}$. It may be that all $\mathcal{N}=2$ supersymmetric gauge theories in four dimensions are related to Hitchin systems with suitable singularities included. At any rate, we will continue the discussion with this important class of examples in mind. When formulated as fourdimensional gauge theories, these theories have a generalized quiver structure.

In what sense might one quantize the completely integrable system $\mathcal{M}_{H}$ ? An important property of $\mathcal{M}_{H}$ is that it is birational to a cotangent bundle $T^{*} \mathcal{M}$, where $\mathcal{M}$ is the moduli 
space of stable holomorphic $G$-bundles on $C$. Such a "birational" equivalence holds after deleting complex submanifolds on both sides. The holomorphic symplectic form of $\mathcal{M}_{H}$ maps to the standard one on $T^{*} \mathcal{M}$.

This equivalence means that one can hope to map the commuting Hamiltonians of the integrable system - in other words, the polynomial functions on $\boldsymbol{B}$ - to holomorphic differential operators on $\mathcal{M}$. This was first done for rank 2 Higgs bundles (in other words, for the case that the gauge group of the generalized quiver is a product of $S U(2)$ 's) in [32] and in generality in [33]; these constructions were based on conformal field theory on $C$. An alternative argument starting from four-dimensional gauge theory was explained at the end of section 11.1 of [13]. An important fact is that the differential operators in question act not on functions but on sections of $K^{1 / 2}$, where $K$ is the canonical line bundle of $\mathcal{M}$. They commute with each other, just like the underlying classical Hamiltonians.

For illustration, let us suppose that $\mathcal{M}$ is of complex dimension 1, which happens for rank 2 if $C$ is a Riemann surface of genus 1 with one marked point. (This corresponds to an $\mathcal{N}=2$ system that is known as the $\mathcal{N}=2^{*}$ theory, whose Coulomb branch was related to the corresponding Hitchin system in [31]. It is also the example considered in [6].) Consider a holomorphic function $f$ on $\mathcal{M}_{H}$ that when restricted to the cotangent bundle $T^{*} \mathcal{M}$ is quadratic on each fiber of the cotangent bundle. (Such a function can be derived from a Beltrami differential on $C$; in gauge theory with a product of $\mathrm{S} U(2)$ gauge groups, it corresponds to a linear combination of the usual order parameters of the Coulomb branch.) Letting $z$ be a local complex coordinate on $\mathcal{M}$, and $p$ a fiber coordinate, $f$ is of the form $f(z, p)=a(z) p^{2}$. In deformation quantization, $p$ maps to $\mathrm{d} / \mathrm{d} z$ so $f$ will map to a second order holomorphic differential operator $\mathcal{D}_{f}$ on $C$. Picking a local trivialization of $K^{1 / 2}, \mathcal{D}_{f}$ is concretely given by a formula

$$
\mathcal{D}_{f}=a(z) \frac{\mathrm{d}^{2}}{\mathrm{~d} z^{2}}+b(z) \frac{\mathrm{d}}{\mathrm{d} z}+c(z)
$$

with local holomorphic functions $a, b, c$. Here, $a$ can be read off from the classical function $f=a(z) p^{2}$, but the usual quantum mechanical problem of operator ordering affects $b$ and $c$. In principle, $b$ and $c$ can be computed in $\sigma$-model perturbation theory for the brane system described in section 2.3. It is a non-trivial fact that unique $b$ and $c$ functions do exist such that the operator $\mathcal{D}_{f}$ is a globally-defined holomorphic differential operator acting on sections of $K^{1 / 2}$. (This is not true if $K^{1 / 2}$ is replaced by any other line bundle.) For various explanations, see $[32,33]$, or section 11 of $[13]$. The details are anyway not really pertinent to the qualitative remarks we will make here.

This construction has been formally called quantization, but it is not what physicists usually mean by quantization. A holomorphic differential operator $\mathcal{D}_{f}$ is constructed, but there is no Hilbert space that it acts on. Indeed, what could such a Hilbert space be? $\mathcal{D}_{f}$ could act on global holomorphic sections of $K^{1 / 2}$, but there are none. $\mathcal{D}_{f}$ could also act on global $C^{\infty}$ sections of $K^{1 / 2}$, or on meromorphic sections of $K^{1 / 2}$ with prescribed poles or with arbitrary poles. But none of these spaces is a Hilbert space in a natural way.

To get a Hilbert space, we should pick ${ }^{1}$ a closed curve $\gamma \in \mathcal{M} . K^{1 / 2}$ restricts on $\gamma$ to the bundle of complex-valued half-densities on $\gamma$, and these form a natural Hilbert space

\footnotetext{
${ }^{1}$ The actual construction made in [6] is more complicated than we are about to explain. The reason is that in their example, $\mathcal{M}_{H}$ is only birational to $T^{*} \mathcal{M}$, for a Riemann surface $\mathcal{M}$. If one aproximates $\mathcal{M}_{H}$
} 
$\mathcal{H}_{\gamma} . \mathcal{D}_{f}$ acts naturally on $\mathcal{H}_{\gamma}$ (as an unbounded operator that is densely defined) for the following reason. Real-analytic sections of $K^{1 / 2}$ along $\gamma$ form a dense subspace $W_{\gamma} \subset \mathcal{H}_{\gamma}$. By definition, a real-analytic section $s$ of $K^{1 / 2}$ along $\gamma$ can be extended to a holomorphic section of $K^{1 / 2}$ on a small neighborhood of $\gamma$ in $\mathcal{M}$. $\mathcal{D}_{f}$ then acts naturally on $s$, and the restriction of $\mathcal{D}_{f} s$ to $\gamma$ is again in $W_{\gamma}$. So $\mathcal{D}_{f}$ acts naturally on the dense subspace $W_{\gamma}$ of the Hilbert space $\mathcal{H}_{\gamma}$. Under suitable hypotheses on $\gamma, \mathcal{D}_{f}$ will be an elliptic operator with a discrete spectrum.

Thus, the function $f$ has been mapped to an operator $\mathcal{D}_{f}$ that acts on a Hilbert space $\mathcal{H}_{\gamma}$. This is what we usually mean by quantization, and it is what is meant by quantization in [6]. However, quantization in this physical sense is not a property of the complex integrable system $\mathcal{M}_{H}$ alone. It depends on a choice of the real curve $\gamma$. In fact, in [6], two different choices of $\gamma$ are considered. (We will derive these two choices from gauge theory in section 3.4.)

In an $n$-dimensional example, the analog of $\gamma$ is an $n$-dimensional real subspace $N \subset$ $\mathcal{M}$. (A subspace $N$ is called real if letting $T N$ be its tangent bundle and $I$ the complex structure of $\mathcal{M}$, one has $T N \cap I(T N)=0$. This implies that $\mathcal{M}$ is a sort of complexification of $N$.) Let $L \subset \mathcal{M}_{H}$ be a completion of $T^{*} N \subset T^{*} \mathcal{M}$. Hitchin's system restricted to $L$ is an ordinary real integrable system, and the construction above can be regarded as quantization in the ordinary sense of this integrable system.

What we have described is a two-step process. Hitchin's integrable system can be quantized at a formal level by the construction of certain holomorphic differential operators. To get an actual Hilbert space requires a choice of a real cycle $N \subset \mathcal{M}$. We have described this for two reasons: to orient the reader to the sense in which we aim to quantize $\mathcal{M}_{H}$, and also to help motivate the two-step nature of the brane construction to which we turn next. In the brane construction, the formal quantization is associated with a single brane, the coisotropic $A$-brane that in the context of the $\Omega$-deformation we will call $\mathcal{B}_{\mathcal{\varepsilon}}$. Construction of an actual Hilbert space $\mathcal{H}$ depends on the choice of a second brane, a Lagrangian $A$ brane $\mathcal{B}_{L}$.

\subsection{Coisotropic $A$-branes}

The brane construction that we will need in this paper relies on aspects of the twodimensional topological $A$-model that are not novel [10-14] but are perhaps also not well known. We will here summarize the facts that will be used later in the paper, without attempting full explanations.

Consider the $A$-model of a symplectic manifold $X$ with symplectic structure $\omega$. An $A$-brane of the familiar sort is supported on a Lagrangian submanifold $L \subset X$ that is endowed with a flat vector bundle. Such an $L$ automatically has half the dimension of $X$. However, in general the $A$-model admits additional branes known as "coisotropic" branes. (One reason that such branes are not well known is that they do not arise for Calabi-Yau threefolds.) The support of such an $A$-brane is a submanifold $Y \subset X$ whose dimension exceeds half of the dimension of $X$.

as $T^{*} \mathcal{M}$, one introduces a bad point in $\mathcal{M}$ where the Hamiltonians have poles. The curves $\gamma$ considered in [6] pass through the bad point and this causes the definition of the Hilbert space to be more involved. 
The most basic new case is the case that $Y=X$. This case will suffice in the present paper. (The general case is a sort of hybrid of this with the more familiar Lagrangian $A$-branes.) Unlike Lagrangian $A$-branes, whose Chan-Paton bundle is flat, the curvature $F$ of a coisotropic $A$-brane is necessarily nonzero. Only the rank 1 case is understood; in this case $F$ is an ordinary two-form. The condition for a rank 1 brane whose support is precisely $X$ and whose Chan-Paton bundle has curvature $F$ to be an $A$-brane is [10] that the linear transformation of the tangent bundle defined by $\mathcal{I}=\omega^{-1} F$ should obey $\mathcal{I}^{2}=-1$. It is then automatically true that $\mathcal{I}$ is an integrable complex structure on $X$. The condition $\mathcal{I}^{2}=-1$ implies that $F$ is not only nonzero but is in fact non-degenerate. To generalize this to include a $B$-field, we simply replace $F$ by $F+B$.

Reading this construction backwards, $X$ is a complex manifold and $\Omega=F+i \omega$ is a holomorphic (2,0)-form that is closed and non-degenerate. Thus, $X$ is a complex symplectic manifold. However, neither the complex structure of $X$ nor $F=\operatorname{Re} \Omega$ are part of the definition of the $A$-model of $X$; rather they were used to define a brane. Only $\omega=\operatorname{Im} \Omega$ is used in defining the $A$-model of $X$. The same $X$ may have many different structures of complex symplectic manifold (not related to each other by exact symplectomorphisms with respect to $\omega$, which are trivial in the $A$-model) each with a holomorphic two-form whose imaginary part is $\omega$ and whose real part is the curvature of some line bundle. Each of these will lead to a different brane in the same $A$-model of $X$. Concrete examples can be constructed in the hyper-Kahler case of section 2.3.1.

Though not yet part of the standard toolkit of physicists, coisotropic $A$-branes have very interesting properties. If $\mathcal{B}$ is a Lagrangian $A$-brane, supported on a Lagrangian submanifold $L$ and endowed with a flat bundle $E$, then the space of $(\mathcal{B}, \mathcal{B})$ strings is ordinarily finite-dimensional and not really quantum mechanical in nature. Additively, it is the cohomology of $L$ with values in the bundle $E \otimes E^{*}$ ( $E^{*}$ is the dual bundle to $E$ ).

By contrast, if $\mathcal{B}$ is a rank 1 coisotropic $A$-brane whose support is $X$, then the space of $(\mathcal{B}, \mathcal{B})$ strings of fermion number zero is additively the space of complex-valued functions on $X$ that are holomorphic in complex structure $\mathcal{I}$; allowing the fermion number to vary, the space of $(\mathcal{B}, \mathcal{B})$ strings is the $\bar{\partial}$ cohomology of $X$. This is quite an unusual answer; we are accustomed to holomorphic functions and $\bar{\partial}$ cohomology in the $B$-model, but not in the $A$-model. Of course, the size of the space of $(\mathcal{B}, \mathcal{B})$ strings depends very much on $X$. At one extreme, if $X$ is compact, the only global holomorphic functions are constants. We will be interested in cases in which $X$ admits many holomorphic functions. The extreme case is the case that $X$ is an affine variety, admitting in a sense as many global holomorphic functions as there are on $\mathbb{C}^{n}$. (In our applications, $X$ will or will not have this property depending on the precise choice of coisotropic $A$-brane.)

Even more remarkable is the ring structure that arises from the joining of $(\mathcal{B}, \mathcal{B})$ strings (or equivalently, from the multiplication of boundary vertex operators that represent such strings). This ring is a noncommutative deformation of the ring of holomorphic functions on $X$ (or its extension to include the higher $\bar{\partial}$ cohomology). In the case that $X$ has lots of holomorphic functions, the noncommutative $\operatorname{ring} \mathcal{R}$ of $(\mathcal{B}, \mathcal{B})$ strings is the ring that can be obtained by deformation quantization of the ring of holomorphic functions with respect to the holomorphic symplectic form $\Omega$. In other words, the first order departure 
from commutativity is given by the Poisson bracket $\{f, g\}=\left(\Omega^{-1}\right)^{i j} \partial_{i} f \partial_{j} g$, with higher order corrections largely determined by associativity of the operator product expansion. See [11] or in more detail [13], section 11; see also [28] for a related analysis.

To understand the noncommutative structure of the $\operatorname{ring}$ of $(\mathcal{B}, \mathcal{B})$ strings, recall that, in general, interactions of open strings involve a noncommutative structure that is much more complicated than deformation quantization of a finite-dimensional manifold. It is exceptional to find a situation in which noncommutativity survives but reduces to something as simple as ordinary deformation quantization. This occurs in somewhat similar ways in the presence of a strong $B$-field $[29,30]$ or in the $A$-model with a coisotropic brane; either the strong $B$-field or the topological symmetry of the $A$-model can eliminate most of the open string modes, reducing to a finite-dimensional but still noncommutative story. Let us briefly describe how this comes about.

Suppose that on the target space $X$ of a sigma-model we have a metric $g$ and a $B$-field $B$. We consider a brane endowed with Chan-Paton curvature $F$. We make no assumption in general that $X$ is a complex manifold, and write $I, J=1, \ldots, n$ for tangent space indices to $X$. In sigma-model, one encounters [30] an effective inverse metric

$$
G^{I J}=\left(\frac{1}{g+2 \pi \alpha^{\prime}(F+B)} g \frac{1}{g-2 \pi \alpha^{\prime}(F+B)}\right)^{I J}
$$

and a noncommutativity parameter

$$
\theta^{I J}=-\left(2 \pi \alpha^{\prime}\right)^{2}\left(\frac{1}{g+2 \pi \alpha^{\prime}(F+B)}(F+B) \frac{1}{g-2 \pi \alpha^{\prime}(F+B)}\right)^{I J} .
$$

In sigma-model perturbation theory, in computing the operator product expansion of boundary operators $\mathcal{O}_{u}$ and $\mathcal{O}_{v}$ associated to functions $u$ and $v$ on $X$, one meets symmetric contractions proportional to $G^{I J} \partial_{I} u \partial_{J} v$, and antisymmetric contractions proportional to $\theta^{I J} \partial_{I} u \partial_{J} v$. For the sigma-model to reduce to something as simple as deformation quantization, the symmetric contraction must be negligible compared to the antisymmetric one. The most familiar way to achieve this result is to take $F+B \rightarrow \infty$, noting that $G^{I J} \sim 1 /(F+B)^{2}$ while $\theta^{I J} \sim 1 /(F+B)$. However, in the $A$-model, there is another way to suppress the symmetric contractions. In the context of the coisotropic $A$-brane $\mathcal{B}$ just described, $u$ and $v$ are required to be holomorphic functions in complex structure $\mathcal{I}$. Moreover, $g$ is of type $(1,1)$ while $F+B$ is of type $(2,0) \oplus(0,2)$. In this case, the symmetric contraction vanishes, and the antisymmetric contraction is governed by $\theta^{-1}=-(F+B)_{2,0}^{-1}$, that is, the $(2,0)$ part of $-(F+B)^{-1}$. In our above presentation, we took $B=0, F=\operatorname{Re} \Omega$, so the anticommutativity parameter is $-\Omega^{-1}$.

Now the question arises of whether we can use this framework to see quantum mechanics, and not simply deformation quantization. To do so, we need a Hilbert space on which the noncommutative ring $\mathcal{R}$ acts. For this, we consider a pair of $A$-branes - a coisotropic $A$-brane $\mathcal{B}$ of support $X$ and a rank 1 Lagrangian $A$-brane $\mathcal{B}_{L}$ of support $L$. We write $\mathcal{L}$ and $\mathcal{S}$ for the Chan-Paton bundles of $\mathcal{B}$ and $\mathcal{B}_{L}$, respectively. $\mathcal{L}$ and $\mathcal{S}$ are both endowed with unitary connections. The curvature of $\mathcal{L}$ is a non-degenerate two-form $F$; the curvature of $\mathcal{S}$ vanishes. Whatever $L$ is, the $\operatorname{ring} \mathcal{R}$ of $(\mathcal{B}, \mathcal{B})$ strings will act on 
the space $\mathcal{H}$ of $\left(\mathcal{B}, \mathcal{B}_{L}\right)$ strings, by the usual operation of joining strings. The fact that we must introduce a second brane to define $\mathcal{H}$ has an obvious parallel with what we stated more naively in section ${ }^{2}$ 2.2. Under certain conditions which we will now state, $\mathcal{H}$ can be interpreted in terms of quantization of $L$.

The fact that $L$ is Lagrangian means by definition that $\omega$ vanishes when restricted to $L$. What about $F$ ? Let us consider two contrasting cases. (There are also various intermediate cases, but they will not be important for us.) If $F$ vanishes when restricted to $L$, then $L$ is actually a complex submanifold ${ }^{3}$ in complex structure $\mathcal{I}$. This case does not lead to what physicists usually understand as quantization, but can lead to interesting and purely holomorphic constructions of spaces on which $\mathcal{R}$ acts, and it is important for geometric Langlands [13].

The opposite case is the case that $F$ remains nondegenerate when restricted to $L$. Thus, though Lagrangian with respect to $\omega, L$ is symplectic with respect to $F$. If unitarity is desired, one also requires that $X$ should have an antiholomorphic involution (a symmetry of order 2) with $L$ as a component of its fixed point set. Under these conditions, as found in [12] in examples and discussed more systematically in [14], the space $\mathcal{H}$ of $\left(\mathcal{B}, \mathcal{B}_{L}\right)$ strings can be understood as a quantization of $L$, with symplectic structure $F$ (and prequantum line bundle $\mathcal{L} \otimes \mathcal{S}^{-1}$, whose curvature is $F$ ). The basic reason for this is easily explained. As usual, the physical states of the $A$-model are the string ground states, which can be found by quantizing the zero-modes of the string. In the case of the $\left(\mathcal{B}, \mathcal{B}_{L}\right)$ strings, one finds that there are no fermion zero-modes. The bosonic zero-modes describe the motion of the string along $L$, and the relevant part of the action for these modes is the Chan-Paton contribution. Writing $\boldsymbol{A}=\sum_{i} p_{i} \mathrm{~d} q^{i}$ for the connection on the prequantum line bundle $\mathcal{L} \otimes \mathcal{S}^{-1}\left(p_{i}\right.$ and $q^{j}$ are a local system of canonically conjugate coordinates on $L$ viewed as a symplectic manifold with symplectic form $\left.\omega_{J}\right)$, the relevant action is $\int \mathrm{d} t p_{i} \mathrm{~d} q^{i} / \mathrm{d} t$; quantization of the zero-modes with this action is usually called quantization of $L$.

The functions on $L$ that can be most naturally quantized as operators on $\mathcal{H}$ are the functions that are restrictions to $L$ of holomorphic functions on $X$. Such functions are quantized by identifying them with $(\mathcal{B}, \mathcal{B})$ strings which then act naturally on $\mathcal{H}$.

If $X$ is an affine variety in complex structure $\mathcal{I}$, functions on $L$ that are restrictions of holomorphic functions on $X$ are dense in the space of functions on $L$. We consider two primary applications in this paper. In section 4, where we discuss the relation [15] between four-dimensional gauge theory and two-dimensional Liouville theory, $X$ is indeed an affine variety in complex structure $\mathcal{I}$. The holomorphic functions on $X$ are numerous and act irreducibly in the quantization. In section 3, where we discuss the relation [6] of the

\footnotetext{
${ }^{2}$ In our presentation, we are eliding a few key details that are described in [14]. Though $\mathcal{H}$ can always be defined in the $A$-model, and has a Hilbert space structure because it is the space of ground states of the sigma-model, this Hilbert space structure is natural in the $A$-model only if $L$ is the fixed point set of an antiholomorphic involution of $X$. And when this is the case, the Hilbert space structure on $\mathcal{H}$ that is natural in the $A$-model coincides with the naive one introduced in section 2.2 only in the semiclassical limit, that is, to lowest order in sigma-model perturbation theory.

${ }^{3}$ For a proof of this statement, see section 2.4 - though a different notation is used there with $I, J$, and $K$ cyclically permuted. As will become clear in this paper, it is difficult to find a single and uniformly convenient notation.
} 
$\Omega$-deformation to quantization of Hitchin's integrable system, $X$ is not affine in complex structure $\mathcal{I}$ (but instead is a fibration by abelian varieties over an affine base $\boldsymbol{B}$ ). The holomorphic functions are the commuting Hamiltonians of Hitchin's integrable system. Their interpretation via $(\mathcal{B}, \mathcal{B})$ strings amounts to their interpretation as commuting holomorphic differential operators on $\mathcal{M}$, as explained in detail in section 11 of [13]. To get a Hilbert space $\mathcal{H}$ on which these operators can act, we need to pick a real section of $\mathcal{M}$, as explained heuristically in section 2.2. More fundamentally, we need to pick a Lagrangian brane $\mathcal{B}_{L}$ in the $A$-model of $\mathcal{M}_{H}$ in symplectic structure $\omega_{K}$.

Our two applications will involve different sides of the same coin. But to explain this, we must now specialize to the hyper-Kahler situation.

\subsubsection{The Hyper-Kahler case}

In our examples, $X$ will be the Coulomb branch of the moduli space of vacua of a fourdimensional theory with $\mathcal{N}=2$ supersymmetry, after compactification on a circle (or a two-torus) to three or two dimensions. Thus $X$ will be hyper-Kahler. An important class of examples [16], already considered for illustration above, are the generalized quiver theories in which $X$ is a moduli space $\mathcal{M}_{H}$ of Higgs bundles on a Riemann surface $C$.

Such an $X$ has a distinguished complex structure $I$ (in which the Hitchin fibration is holomorphic) and another distinguished complex structure $J$ (in which it parametrizes complex-valued flat connections on $C$ ). We set $K=I J ; I, J$, and $K$ obey the quaternion algebra. Together with a Riemannian metric $g$, they define the hyper-Kahler structure of $X$. Any linear combination $\mathcal{I}=a I+b J+c K$, where $a, b, c$ are real and $a^{2}+b^{2}+c^{2}=1$, is an integrable complex structure. This family of complex structures is parametrized by $\mathbb{C P}^{1} \cong S^{2}$. The three real symplectic forms, which are Kahler in complex structures $I, J$, or $K$ respectively, are $\omega_{I}=g I, \omega_{J}=g J, \omega_{K}=g K$. Similarly, the three holomorphic symplectic forms, which are of type $(2,0)$ with respect to $I, J$, or $K$, are $\Omega_{I}=\omega_{J}+i \omega_{K}$, $\Omega_{J}=\omega_{K}+i \omega_{I}$, and $\Omega_{K}=\omega_{I}+i \omega_{J}$. For simplicity, we will assume the $B$-field to vanish.

We will be studying the $A$-model of $X$ in the symplectic structure $\omega=\omega_{K}$. To define a coisotropic brane in this situation, we can take $F=\omega_{I} \sin p+\omega_{J} \cos p$ for some angle $p$. We must constrain $p$ and the hyper-Kahler metric of $X$ so that $F / 2 \pi$ has integer periods and hence $F$ is the curvature of some line bundle $\mathcal{L} \rightarrow X$. Given this, we get a coisotropic $A$-brane with $\mathcal{I}=\omega^{-1} F=I \cos p-J \sin p$.

For our eventual application, the most important case will be that $p=0$, so $F=\omega_{J}$ and $\mathcal{I}=I$. (In examples arising by compactification from $\mathcal{N}=2$ theories in four dimensions, $\omega_{J}$ is cohomologically trivial, so there is no problem in constructing a line bundle with curvature $F$.) The brane $\mathcal{B}$ so obtained is a sufficiently basic example of a coisotropic $A$ brane that it has been called the canonical coisotropic $A$-brane $\mathcal{B}_{\mathrm{cc}}$. We have constructed it to be an $A$-brane in the $A$-model of symplectic structure $\omega_{K}$. However, it has additional supersymmetric properties and these will be important. Suppose we take $\omega=\omega_{I}$; then

$\omega^{-1} F=-K$, which is again an integrable complex structure. So the same brane $\mathcal{B}_{\text {cc }}$ is also an $A$-brane for another $A$-model, the one with $\omega=\omega_{I}$. Finally, as $\omega_{J}$ is of type $(1,1)$ in complex structure $J$, we see that $\mathcal{B}_{\text {cc }}$ is a $B$-brane for the $B$-model of complex structure $J$. 
These three facts are related, in the following sense. The topological supercharges of the $A$-model of symplectic structure $\omega_{I}$, the $B$-model of complex structure $J$, and the $A$-model of symplectic structure $\omega_{K}$ obey one linear relation. They are linear combinations of two supercharges $Q$ and $Q^{\prime}$ (this will be explained in detail in section 3). Any linear combination $u Q+v Q^{\prime}$, with complex coefficients $u, v$ that are not both zero, squares to zero and is the topological supercharge of some topological field theory. Varying the ratio $u / v$, this gives a family of topological field theories, parametrized by $\mathbb{C P}^{1}$, which admit the same brane $\mathcal{B}_{\mathrm{cc}}$. For brevity, we will describe this by saying that $\mathcal{B}_{\mathrm{cc}}$ is a brane of type $(A, B, A)$.

As branes with multiple supersymmetric properties may be unfamiliar, we will mention a much more obvious example that will also be important in this paper. This is the brane $\mathcal{B}_{*}$ whose support is all of $X$ and whose Chan-Paton line bundle $\mathcal{L}_{*}$ is trivial. The support of $\mathcal{B}_{*}$ (being all of $X$ ) and the line bundle $\mathcal{L}_{*}$ (being flat) are both holomorphic in every complex structure $\mathcal{I}=a I+b J+c K$. So the brane $\mathcal{B}_{*}$ is a $B$-brane in a family of $B$-models parametrized by $\mathbb{C P}^{1}$. We summarize this by saying that $\mathcal{B}_{*}$ is a brane of type $(B, B, B)$. Again, the various supercharges are linear combinations of any two of them.

The differential geometry of branes with multiple supersymmetric properties is further described in section 2.4 .

\subsubsection{First among equals}

In this hyper-Kahler situation, we can define a plethora of topological field theory structures - the $B$-model in any complex structure $a I+b J+c K$, or the $A$-model in any symplectic structure $a \omega_{I}+b \omega_{J}+c \omega_{K}$. However, in the important case [16] that $X$ is actually a moduli space $\mathcal{M}_{H}$ of Higgs bundles on a Riemann surface $C$, with some gauge group $G$, some of these structures are more special than others.

Almost all of these two-dimensional topological field theories depend on the complex structure of $C$. But some do not. In one of its complex structures, customarily called $J$, $\mathcal{M}_{H}$ is the moduli space of flat connections on $C$ with values in the complexification of $G$. This is a complex symplectic manifold in a completely natural way, independent of any choice of metric or even complex structure on $C$. Thus, both the complex structure $J$ and the holomorphic two-form $\Omega_{J}=\omega_{K}+i \omega_{I}$ do not depend on any property of $C$ beyond its orientation. In fact, $\Omega_{J}$ can be defined by the formula

$$
\Omega_{J}=\frac{i}{4 \pi} \int_{C} \operatorname{Tr} \delta \mathcal{A} \wedge \delta \mathcal{A}
$$

where $\mathcal{A}=A+i \phi$ is a complex-valued flat connection; this formula does not use a metric or complex structure on $C$, so it makes clear the topological nature of $\Omega_{J}$.

Accordingly, the $B$-model of type $J$ and the $A$-models of types $\omega_{I}$ and $\omega_{K}$ are special - they do not depend on the choice of a complex struture on $C$. In that sense, branes of type $(A, B, A)$ are special, compared to say branes of type $(B, B, B),(B, A, A)$, or $(A, A, B)$, all of which have a mixture of properties that do or do not depend on a complex structure on $C$. 
As explained earlier, a brane on $\mathcal{M}_{H}$ with multiple supersymmetric properties is a brane in a whole family of topological field theories, parametrized by $\mathbb{C P}^{1}$. In general, this family depends on the complex structure of the underlying Riemann surface $C$. Precisely in the case of a brane of type $(A, B, A)$, the relevant family of topological field theories is independent of the complex structure of $C$. Our applications are based on this family, as is also the gauge theory approach to geometric Langlands [13].

In a four-dimensional $\mathcal{N}=2$ theory with $\mathrm{U}(1)_{R}$ symmetry, the forms $\omega_{J}$ and $\omega_{K}$ are rotated by the $\mathrm{U}(1)_{R}$ symmetry, which ensures that their cohomology classes (which would have to be rotation-invariant) must vanish. This is actually true even without $\mathrm{U}(1)_{R}$ symmetry, as long as hypermultiplet bare masses vanish. For generalized quiver theories associated to a Riemann surface $C$ without marked points, this can be shown in terms of differential geometry as follows. Describing a Higgs bundle by a gauge field $A$ with Higgs field $\phi$, the exactness of $\omega_{J}$ and $\omega_{K}$ follows from an explicit formula

$$
\omega_{J}+i \omega_{K}=\delta\left(\frac{1}{\pi} \int_{C} \operatorname{Tr} \phi_{z} \delta A_{\bar{z}}\right) .
$$

(In the presence of a hypermultiplet bare mass, $\phi_{z}$ has a pole whose residue has an eigenvalue proportional to the mass, and the above argument fails because the one-form in parentheses is not gauge-invariant; that is, it is not the pullback of a one-form on $\mathcal{M}_{H}$.) However, the form $\omega_{I}$ is topologically non-trivial. It is given by the imaginary part of (2.16), or

$$
\omega_{I}=\frac{1}{4 \pi} \int_{C} \operatorname{Tr}(\delta A \wedge \delta A-\delta \phi \wedge \delta \phi) .
$$

One way to prove that $\omega_{I}$ has a non-zero cohomology class is to observe that, when restricted to the locus $\phi=0$, it becomes the Kahler form of the compact Kahler manifold $\mathcal{M} \subset \mathcal{M}_{H}$ that parametrizes flat $G$-bundles on $C$. The Kahler form of a compact Kahler manifold always has a non-trivial cohomology class.

Now let us discuss what two-dimensional $A$-models we can make using the symplectic forms $\omega_{I}$ and $\omega_{K}$ that do not depend on a choice of complex structure on $C$. (We keep away from $\omega_{J}$, since it does depend on the complex structure of $C$.) Superficially, we can introduce two complex parameters $\boldsymbol{u}, \boldsymbol{v}$, since the complexified Kahler class $\widehat{\omega}$ used in defining the $A$-model may be any complex linear combination

$$
\widehat{\omega}=\boldsymbol{u} \omega_{I}+\boldsymbol{v} \omega_{K}
$$

We must take the real parts of $\boldsymbol{u}$ and $\boldsymbol{v}$ to be not both zero, because to define an $A$-model, Re $\widehat{\omega}$ must be a symplectic form. However, in the absence of hypermultiplet bare masses, because $\omega_{K}$ is exact, there is really only one complex parameter that matters, namely $\boldsymbol{u}$. If $\operatorname{Re} \boldsymbol{u}=0$, we must take $\operatorname{Re} \boldsymbol{v} \neq 0$, but its magnitude and even sign are not relevant. (The sign of Re $\boldsymbol{v}$ can be reversed by a rotation of the Higgs field.) So really the only meaningful parameter is $\boldsymbol{u}$. Arbitrary values of $\boldsymbol{u} \in \mathbb{C}$ make sense, with $\boldsymbol{v}$ turned on if necessary (or if desired). There is actually also a limit as $\boldsymbol{u} \rightarrow \infty$; this limit is the $B$-model in complex structure $J$. The fact that the limit of $A$-models for $\widehat{\omega} \rightarrow \infty$ exists and is a $B$-model 
is most readily understood using generalized complex geometry and will be reviewed in section 2.4.2.

Both in interpreting geometric Langlands duality via gauge theory [13] and in section 3 of the present paper, the important special case of the $A$-model is the case $\boldsymbol{u}=0$. This is naturally called the $A$-model of type $\omega_{K}$; as we have explained, in an important situation, this model has no Kahler parameter. In an extended version of geometric Langlands duality, analyzed in section 11 of [13] and encountered in section 4 of the present paper, one requires the generic model with variable $\boldsymbol{u}$. This model is naturally called the $A$-model of type $\omega_{I}$, and of course, it does always have a Kahler parameter, namely $\boldsymbol{u}$.

We will make one last comment on branes of type $(A, B, A)$. Although the conditions that characterize a brane of type $(A, B, A)$ do not depend on a choice of complex structure on $C$, a particular $(A, B, A)$-brane might be defined in a way that does depend on that complex structure. Indeed, we have already discussed a very important example. Given a complex structure on $C, \mathcal{M}_{H}$ becomes hyper-Kahler, and in particular it acquires a symplectic form $\omega_{J}$ that is Kahler with respect to $J$. Unlike $\omega_{I}$ and $\omega_{K}, \omega_{J}$ does depend on the metric of $C$ (as do $I=\omega_{I}^{-1} \omega_{J}$ and $K=\omega_{K}^{-1} \omega_{J}$ ). So the canonical coisotropic $(A, B, A)$ brane does depend on the complex structure of $C$, though it is a brane in a family of topological field theories that do not depend on this metric.

\subsection{A little differential geometry}

On a hyper-Kahler manifold $X$, we have described some branes with multiple supersymmetric properties - the brane $\mathcal{B}_{*}$ is of type $(B, B, B)$, and its cousin $\mathcal{B}_{\mathrm{cc}}$ is of type $(A, B, A)$. These are not the only examples. Another simple example of a brane of type $(B, B, B)$ is a brane supported at a point in $X$ - since a point is a complex submanifold in any complex structure. The most obvious branes of type $(A, B, A)$ are Lagrangian branes of this type. Such a brane is supported on a middle-dimensional submanifold $L \subset X$ that is holomorphic in complex structure $J$, and is Lagrangian for the holomorphic symplectic form $\Omega_{J}=\omega_{K}+i \omega_{I}$. As $L$ is Lagrangian for both $\omega_{K}$ and $\omega_{I}$, a brane supported on $L$ with vanishing Chan-Paton curvature is an $A$-brane of these types; as $L$ is holomorphic in complex structure $J$, such a brane is of type $(A, B, A)$.

The structures that we have described are redundant, in the following sense. A brane that is (for example) a $B$-brane of type $I$ and a $B$-brane of type $J$ is automatically a $B$-brane of type $K$ (and more generally, a $B$-brane in any complex structure $a I+b J+c K$, $a^{2}+b^{2}+c^{2}=1$ ), since the conserved supercharges of these three $B$-models are linearly dependent. This linear dependence will become very clear in section 3 , but here we will briefly explain the redundancy among the different supersymmetric structures from the point of view of differential geometry.

In a hyper-Kahler manifold $X$, consider a brane $\mathcal{B}$ with support $L \subset X$. One condition for $\mathcal{B}$ to be a $B$-brane for complex structures $I$ and $J$ is that $L$ must be holomorphic in those complex structures. If so, then $L$ is also holomorphic in complex structure $K=I J$. Indeed, if the tangent space to $L$ is invariant under the endomorphisms of the tangent bundle to $X$ corresponding to $I$ and $J$, it is certainly invariant under $K=I J$. The other condition for $\mathcal{B}$ to be a $B$-brane for complex structures $I$ and $J$ is that the Chan-Paton 
curvature $F$ is of type $(1,1)$ with respect to both $I$ and $J$; equivalently, $I^{t} F I=J^{t} F J=F$. Clearly this implies that $K^{t} F K=F$, completing the argument that $\mathcal{B}$ is a $B$-brane of type $K$ if it is one of types $I$ and $J$.

For an analogous argument for $A$-branes of type $(A, B, A)$, we will consider just the case of a Lagrangian brane $\mathcal{B}$ supported on a middle-dimensional submanifold $L$. We will show that if $\mathcal{B}$ is an $A$-brane for both $\omega_{I}$ and $\omega_{K}$, then it is a $B$-brane in complex structure $J$. (We leave it to the reader to show that if $\mathcal{B}$ is an $A$-brane for $\omega_{I}$ and a $B$-brane for $J$, then it is an $A$-brane for $\omega_{K}$.) Let $T L$ be the tangent bundle to $L$, and let $N^{*} L$ be the subspace of $\left.T^{*} X\right|_{L}$ (the restriction to $L$ of the cotangent bundle of $X$ ) consisting of cotangent vectors that annihilate $T L$. The fact that $L$ is Lagrangian for both $\omega_{I}$ and $\omega_{K}$ means that $\omega_{I}$ establishes an isomorphism from $T L$ to $N^{*} L$, and $\omega_{K}^{-1}$ is an isomorphism from $N^{*} L$ to $T L$. So $J=\omega_{K}^{-1} \omega_{I}$ is an isomorphism from $T L$ to itself, and thus $L$ is holomorphic in complex structure $J$ and $\mathcal{B}$ is a $B$-brane.

In particular (though we have only shown this for Lagrangian branes) there is no such thing as a brane of type $(A, A, A)$ - if $\mathcal{B}$ is an $A$-brane of type $I$ and $K$, then it is a $B$-brane of type $J$. To illuminate the last statement further, and for some further applications, we will give an overview of the possible half-BPS supersymmetry conditions for a brane on a hyper-Kahler manifold $X$.

\subsubsection{General Half-BPS condition}

On $X$, there is a family of complex structures parametrized by $\mathbb{C P}^{1}$; a general element of this family is a complex structure $\mathcal{J}=a I+b J+c K$, with $a, b, c$ real and $a^{2}+b^{2}+c^{2}=1$.

Twisted topological field theories in two dimensions are conveniently constructed by twisting a theory with $(2,2)$ supersymmetry. In general, a sigma-model with target $X$ and $(2,2)$ supersymmetry is constructed [39] in terms of a pair of integrable complex structures $\mathcal{J}_{+}$and $\mathcal{J}_{-}$, which govern right- and left-moving excitations, respectively. They obey a certain compatibility condition which also involves the metric and the curvature $H$ of the $B$-field. Generalized complex geometry [40] leads to the most elegant interpretation of the compatibility condition [41]. We use this viewpoint below.

If $X$ is a hyper-Kahler manifold, $\mathcal{J}_{+}$and $\mathcal{J}_{-}$can be chosen to correspond to arbitrary points in $\mathbb{C P}^{1}$; the compatibility condition is always obeyed, with $H=0$. Hence, the sigma-model with hyper-Kahler target space has a twisted version corresponding to an arbitrary pair $\left(\mathcal{J}_{+}, \mathcal{J}_{-}\right) \in \mathbb{C P}_{+}^{1} \times \mathbb{C P}_{-}^{1}$, that is, in the product of two copies of $\mathbb{C P}^{1}$. A $B$-model corresponds to the case that $\mathcal{J}_{+}=\mathcal{J}_{-}$, and an $A$-model corresponds to the case that $\mathcal{J}_{+}=-\mathcal{J}_{-}$.

A supersymmetric boundary condition preserves the supersymmetries associated to certain pairs $\left(\mathcal{J}_{+}, \mathcal{J}_{-}\right)$, but of course, not all possible pairs. In general, a half-BPS boundary condition preserves the supersymmetries associated with pairs of the form $\mathcal{J}_{-}=h \mathcal{J}_{+}$, where $h \in \mathrm{S} O(3)$ is a rigid rotation of $\mathbb{C P}^{1} \cong S^{2}$ that gives a holomorphic map from $\mathbb{C P}_{+}^{1}$ to $\mathbb{C P}_{-}^{1}$. For example, if $h=1$, we have $\mathcal{J}_{+}=\mathcal{J}_{-}$for all $\mathcal{J}_{+}$. This is the condition for a brane of type $(B, B, B)$. Since the antipodal map on $\mathbb{C P}^{1}$ is not as $\mathrm{S} O(3)$ rotation, it is not possible to have $\mathcal{J}_{-}=-\mathcal{J}_{+}$for all $\mathcal{J}_{+}$, and hence there is no such thing as a brane of type $(A, A, A)$. 
Actually, any $h \in \mathrm{SO}(3)$ leaves fixed some axis in $\mathbb{C P}^{1} \cong S^{2}$, so there is always some choice of $\mathcal{J}_{+}$for which $h \mathcal{J}_{+}=\mathcal{J}_{+}$. Hence any half-BPS brane $\mathcal{B}$ is a $B$-brane in some complex structure $\mathcal{J}=a I+b J+c K$, with $a^{2}+b^{2}+c^{2}=1$. It is not true that there is always some $\mathcal{J}_{+}$with $h \mathcal{J}_{+}=-\mathcal{J}_{+}$. Such a $\mathcal{J}_{+}$exists if and only if $h$ is a $\pi$ rotation around the appropriate axis. If so, then regarding $h$ as a linear transformation of a copy of $\mathbb{R}^{3}$ in which $S^{2} \cong \mathbb{C P}^{1}$ is embedded, $h$ has two eigenvalues -1 , and so a brane associated with such a $h$ is an $A$-brane in two different ways. After a suitable rotation of the coordinate axes (so that $h$ is a rotation around the $J$ axis), such a brane is of type $(A, B, A)$.

\subsubsection{Role of generalized complex geometry}

But what happens if $h$ is a rotation by an angle other than $\pi$ ? In this case, although $\mathcal{B}$ is a $B$-brane in one complex structure, its other supersymmetric properties appear unfamiliar.

To allow for the case of an arbitrary $h$, we consider the general case of a brane $\mathcal{B}$ that conserves a topological supercharge $Q$ associated to a pair of independent complex structures $\mathcal{J}_{+}$and $\mathcal{J}_{-}$for the right-moving and left-moving modes. It turns out [11] that on a hyper-Kahler manifold, the topological field theory associated to a pair $\left(\mathcal{J}_{+}, \mathcal{J}_{-}\right)$with $\mathcal{J}_{+} \neq \mathcal{J}_{-}$can always be reduced to an $A$-model, even if $\mathcal{J}_{+} \neq-\mathcal{J}_{-}$. The reduction is made using the language of generalized complex geometry [40,41]. (The requisite formulas are summarized in section 5.2 of [13], where they are applied to geometric Langlands.)

Rather than defining a topological twist by a pair of complex structures with a metric and $B$-field obeying certain conditions, a useful point of view is that such a twist can be determined by the choice of a generalized complex structure $\mathcal{I}$. A generalized complex structure is a linear transformation $\mathcal{I}$ of $T X \oplus T^{*} X$ (the direct sum of the tangent and cotangent bundles of $X$ ) that obeys $\mathcal{I}^{2}=-1$ as well as a certain integrability condition. A $B$-model associated to a complex structure $J$ (whose transpose we denote as $J^{t}$ ) corresponds to the case that

$$
\mathcal{I}_{J}=\left(\begin{array}{cc}
J & 0 \\
0 & -J^{t}
\end{array}\right) .
$$

The $A$-model with a symplectic structure $\omega$ and zero $B$-field corresponds to the case that

$$
\mathcal{I}_{\omega}=\left(\begin{array}{cc}
0 & -\omega^{-1} \\
\omega & 0
\end{array}\right) .
$$

In general, to turn on a $B$-field, pick a closed two-form $B_{0}$ and set

$$
\mathcal{M}\left(B_{0}\right)=\left(\begin{array}{cc}
1 & 0 \\
B_{0} & 1
\end{array}\right) .
$$

The transformation

$$
\mathcal{I} \rightarrow \mathcal{M}\left(B_{0}\right) \mathcal{I} \mathcal{M}\left(B_{0}\right)^{-1}
$$

is known as a $B$-field transform. It preserves the condition $\mathcal{I}^{2}=-1$ and the integrability condition obeyed by $\mathcal{I}$, and has the effect of shifting the $B$-field by $B_{0}$. In particular, the 
generalization of $\mathcal{I}_{\omega}$ to include a $B$-field is

$$
\mathcal{I}_{\omega, B}=\mathcal{M}(B)\left(\begin{array}{cc}
0 & -\omega^{-1} \\
\omega & 0
\end{array}\right) \mathcal{M}(B)^{-1} .
$$

Now return to the case of a hyper-Kahler manifold $X$ with a pair of complex structures $\mathcal{J}_{ \pm}$for right-movers and left-movers. Denoting as $g$ the hyper-Kahler metric of $X$, let $\omega_{ \pm}=g \mathcal{J}_{ \pm}$be the Kahler forms associated to the complex structures $\mathcal{J}_{ \pm}$. The generalized complex structure associated to this data is, according to eq. 6.3 of [41],

$$
\mathcal{J}=\frac{1}{2}\left(\begin{array}{l}
\mathcal{J}_{+}+\mathcal{J}_{-}-\left(\omega_{+}^{-1}-\omega_{-}^{-1}\right) \\
\omega_{+}-\omega_{-}-\left(J_{+}^{t}+J_{-}^{t}\right)
\end{array}\right) .
$$

(There is also a second generalized complex structure that we do not need here; it is obtained by reversing the sign of $\mathcal{J}_{-}$and $\omega_{-}$.) As long as $\mathcal{J}_{+} \neq \mathcal{J}_{-}$, this takes the form of eq. (2.24) with

$$
\begin{aligned}
\omega^{-1} & =\frac{1}{2}\left(\omega_{+}^{-1}-\omega_{-}^{-1}\right) \\
\omega^{-1} B & =\frac{1}{2}\left(\mathcal{J}_{+}+\mathcal{J}_{-}\right) .
\end{aligned}
$$

Hence, the model is equivalent to an $A$-model.

For our application, an important special case is that $\mathcal{J}_{+}$is very close to $\mathcal{J}_{-}$- so the model is almost a $B$-model. If $\mathcal{J}_{+}-\mathcal{J}_{-}$is of order $\varepsilon$, where $\varepsilon$ is a small parameter, then according to (2.26), $\omega$ and $B$ and therefore the complexified Kahler form $\widehat{\omega}=\omega+i B$ are of order $\varepsilon^{-1}$. This seems a little puzzling because one expects the effects of rotating $\mathcal{J}_{+}$slightly away from $\mathcal{J}_{-}$to be small. However, as we have reviewed in section 2.3, noncommutative effects in the $A$-model are of order $\widehat{\omega}^{-1}$, which in the present context means that these effects are of order $\varepsilon$. In the limit that $\mathcal{J}_{+}$approaches $\mathcal{J}_{-}$, the noncommutative effects in the $A$-model vanish and the $A$-model becomes an ordinary commutative $B$-model.

Since $\widehat{\omega}$ diverges as $\varepsilon \rightarrow 0$, one might not expect the $A$-model with complexified symplectic form $\widehat{\omega}$ to have a limit as $\varepsilon \rightarrow 0$. But in fact this limit exists and is simply the $B$-model of type $\mathcal{J}_{+}$or equivalently $\mathcal{J}_{-}$.

\subsection{3 "Rotation" group}

Two-dimensional topological field theories of the class considered here are labeled by the pair $\left(\mathcal{J}_{+}, \mathcal{J}_{-}\right)$, which parametrize what we may call $\mathbb{C P}_{+}^{1} \times \mathbb{C P}_{-}^{1}$, with one copy of $\mathbb{C P}^{1}$ for $\mathcal{J}_{+}$and one for $\mathcal{J}_{-}$. It is natural to introduce a group $\Gamma=\mathrm{S} U(2)_{+} \times \mathrm{S} U(2)_{-}$that rotates $\mathbb{C P}_{+}^{1} \times \mathbb{C P}_{-}^{1}$, with one factor of $\mathrm{SU}(2)$ for each factor of $\mathbb{C P}^{1}$. The group that acts faithfully on $\mathbb{C P}_{+}^{1} \times \mathbb{C P}_{-}^{1}$ is actually $\mathrm{SO}(3)_{+} \times \mathrm{SO}(3)_{-}$, where $\mathrm{S} O(3)_{ \pm}=\mathrm{S} U(2)_{ \pm} / \mathbb{Z}_{2} . \Gamma$ is a double cover of $\mathrm{SO}(4)=\left(\mathrm{S} U(2)_{+} \times \mathrm{S} U(2)_{-}\right) / \mathbb{Z}_{2}$.

Consider a half-BPS brane characterized by a condition $\mathcal{J}_{-}=h \mathcal{J}_{+}, h \in \mathrm{S} U(2)$. Obviously, if we transform $\left(\mathcal{J}_{+}, \mathcal{J}_{-}\right)$to $\left(g_{+} \mathcal{J}_{+}, g_{-} \mathcal{J}_{-}\right)$, then $h$ is transformed to $h^{\prime}=g_{-}^{-1} h g_{+}$.

For example, suppose that $h=1$, corresponding to a brane of type $(B, B, B)$. Then $h^{\prime}=g_{-}^{-1} g_{+}$. If (as will occur in our application), $g_{+}$is a rotation around some axis by an angle $\vartheta$, and $g_{-}=g_{+}^{-1}$, then $h^{\prime}$ is a rotation around the given axis by an angle $2 \vartheta$. 


\section{Compactification and $\Omega$-deformation}

Finally, we are prepared to consider our first application: the relation of the $\Omega$-deformation to quantization.

The object of study in [6] was a four-dimensional gauge theory with $\mathcal{N}=2$ supersymmetry, "compactified" to two dimensions on $\mathbb{R}_{\varepsilon}^{2}$. Here $\mathbb{R}_{\varepsilon}^{2}$ is simply $\mathbb{R}^{2}$, endowed with a $\mathrm{U}(1)$ rotation symmetry that leaves the origin fixed; the gauge theory on $\mathbb{R}_{\varepsilon}^{2}$ is deformed via the $\Omega$-deformation [7] with parameter $\varepsilon$.

The precise metric on $\mathbb{R}^{2}$ is not essential, as long as it is $\mathrm{U}(1)$-invariant. We will find it helpful to place on $\mathbb{R}^{2}$ a "cigar-like" metric

$$
\mathrm{d} s^{2}=\mathrm{d} r^{2}+f(r) \mathrm{d} \theta^{2}, 0 \leq r<\infty, \quad 0 \leq \theta \leq 2 \pi,
$$

with $f(r) \sim r^{2}$ for $r \rightarrow 0$ and $f(r) \rightarrow \rho^{2}$ for $r \rightarrow \infty$. Thus $\rho$ is the asymptotic radius of the circle parametrized by $\theta$. We can assume that $f(r)$ is identically equal to $\rho$ for sufficiently large $r$ (say $r \geq r_{0}$ ). We write $D$ for $\mathbb{R}^{2}$ endowed with this kind of metric. We also write $D_{R}$ for $D$ restricted to $r \leq R$, where we choose $R$ so that $R \gg \rho, r_{0}$.

We will compactify to two dimensions on $D_{R}$, with an $\Omega$-deformation and a suitable supersymmetric boundary condition at $r=R$. However, first we will need to understand what happens in the absence of the $\Omega$-deformation.

To make contact with the explanation of integrability in section 2.1, we take the twomanifold to which we compactify on $D_{R}$ to be $\mathbb{R} \times S^{1}$. So overall, we will be doing gauge theory on $M=\mathbb{R} \times S^{1} \times D_{R}$. Since we take the cutoff $R$ very large, $D_{R}$ looks macroscopically like $I \times \widetilde{S}^{1}$, where $\widetilde{S}^{1}$ is a second circle, parametrized by $\theta$, and the interval $I$ is parametrized by $r, 0 \leq r \leq R$. Macroscopically, $M$ is a two-torus fibration, that is an $S^{1} \times \widetilde{S}^{1}$ fibration, over $\mathbb{R} \times I$. We should be able to reduce to an effective description in a sigma-model on $\mathbb{R} \times I$.

The appropriate sigma-model is obtained by compactification of our four-dimensional gauge theory to two dimensions on a two-torus $T^{2}$. For orientation, we consider the generalized quiver theories that are obtained [16] by compactifying the six-dimensional $(0,2)$ theory on a Riemann surface $C$, perhaps with surface operators supported at marked points on $C$. In this case, our $\mathcal{N}=2$ gauge theory on $M=\mathbb{R} \times S^{1} \times D_{R}$ will reduce at long distances to the sigma-model on $\Sigma=\mathbb{R} \times I$ with target $\mathcal{M}_{H}$, the moduli space of Higgs bundles on $C$.

To complete this description, we need to specify two branes, supplying boundary conditions at the two ends of $I$. The brane at $r=0$ will in some sense arise purely from geometry, as $r=0$ is not really a boundary point in the more microscopic description on $M$. So one of our questions will be to identify the brane that is generated by geometry. The second boundary condition in the two-dimensional description, the one at $r=R$, will descend from a choice of a boundary condition in the four-dimensional gauge theory.

To account for the results of [6], we want quantization of the sigma-model on $\Sigma=\mathbb{R} \times I$ to give quantization in the ordinary sense of a middle-dimensional real subspace of $\mathcal{M}_{H}$. From section 2.3, we know how this might happen: the effective model on $\Sigma$ should be a two-dimensional $A$-model; one brane should be a canonical coisotropic brane $\mathcal{B}_{\mathrm{cc}}$, with 
support all of $\mathcal{M}_{H}$, while the other should be an ordinary Lagrangian brane $\mathcal{B}_{L}$, with support a Lagrangian submanifold $L \subset \mathcal{M}_{H}$. It will turn out that the brane that arises from geometry will be $\mathcal{B}_{\mathrm{cc}}$, while the Lagrangian brane $\mathcal{B}_{L}$ will depend on a choice of boundary condition at $r=R$.

In section 3.1, we study compactification on $D_{R}$ in the absence of the $\Omega$-deformation. We identify the brane that arises at $r=0$ in the effective two-dimensional description. The support of this brane is all of $\mathcal{M}_{H}$; however, it is not a coisotropic brane, but the more elementary brane $\mathcal{B}_{*}$ of type $(B, B, B)$ described at the end of section 2.3.1. In view of [6], the way to remedy this must be to incorporate the $\Omega$-deformation. In section 3.2 , we reformulate the $\Omega$-deformation in a way suitable for our purposes. In section 3.3 , we consider the $\Omega$-deformed theory on $M=\mathbb{R} \times I \times D_{R}$ and explain why the $\Omega$-deformation has the desired effects. In section 3.4, we describe boundary conditions at the far end of $D_{R}$.

\subsection{The undeformed case}

Two different $2+2$-dimensional splits of $M=\mathbb{R} \times S^{1} \times D_{R}$ will be important in this paper. The first is the obvious decomposition of $M$ as the product of two two-manifolds $\mathbb{R} \times S^{1}$ and $D_{R}$. The second involves using the fact that $D_{R}$ is asymptotic to $I \times \widetilde{S}^{1}$ and viewing $M$ as an $S^{1} \times \widetilde{S}^{1}$ fibration over $\mathbb{R} \times I$. Unfortunately, it is difficult to find a notation that is well-adapted to both decompositions. What we will do is simply to number the coordinates as $0,1,2,3$ for $\mathbb{R}, S^{1}, I$, and $\widetilde{S}^{1}$, respectively.

The bosonic part of the four-dimensional vector multiplet comprises a gauge field $A_{\mu}$, $\mu=0, \ldots, 3$ and a complex scalar $\phi$ in the adjoint representation. It is convenient to adopt a six-dimensional notation in which $A_{\mu}$ and $\phi$ combine to a six-dimensional gauge field $A_{I}, I=0, \ldots, 5$ which is independent of the last two coordinates; from this point of view, $\phi=\left(A_{4}-i A_{5}\right) / \sqrt{2}$. This is useful because, although there is not really an $\mathrm{S} O(6)$ symmetry rotating the six components of $A_{I}$, many key equations can conveniently be written in $\mathrm{SO}(6)$ notation. For example, the supersymmetry generator $\eta$ is a spinor of definite chirality, so if we introduce gamma matrices $\Gamma_{I}, I=0, \ldots, 6$, obeying (in Euclidean signature) $\left\{\Gamma_{I}, \Gamma_{J}\right\}=2 \delta_{I J}$, then

$$
\Gamma_{0} \Gamma_{1} \cdots \Gamma_{5} \eta=i \eta
$$

The fermions $\Psi$ of the vector multiplet are similarly a Weyl spinor in the adjoint representation of the gauge group, with the same chirality as $\eta$. Apart from being chiral spinors of $\mathrm{SO}(6), \eta$ and $\Psi$ are also spinors of the $\mathrm{S} U(2)_{R}$ group of $R$-symmetries. We denote as $\sigma_{i}$, $i=1,2,3$ the analogs for $\mathrm{S} U(2)_{R}$ of the gamma matrices, obeying $\sigma_{i} \sigma_{j}=\delta_{i j}+i \epsilon_{i j k} \sigma_{k}$. We will use standard abbreviations such as $\Gamma_{I J}=\Gamma_{I} \Gamma_{J}, I \neq J$, and $\sigma_{i j}=\sigma_{i} \sigma_{j}=i \epsilon_{i j k} \sigma_{k}, i \neq j$. An example of the usefulness of the six-dimensional notation is that the supersymmetry transformations for the vector multiplet are simply written:

$$
\begin{aligned}
\delta A_{I} & =i \bar{\eta} \Gamma_{I} \Psi \\
\delta \Psi & =\frac{1}{2} \Gamma^{I J} F_{I J} \eta .
\end{aligned}
$$


Now consider an $\mathcal{N}=2$ gauge theory on the four-manifold $M=\mathbb{R} \times S^{1} \times D_{R}$. Away from the tip of the cigar (that is, the region near $r=0$ ), $D_{R}$ is equivalent to $I \times \widetilde{S}^{1}$ and so $M$ reduces to the flat manifold $\mathbb{R} \times S^{1} \times I \times \widetilde{S}^{1}$. On this flat manifold, there are eight unbroken supersymmetries corresponding to all eight components of $\eta$.

The curvature near the tip of the cigar inevitably breaks some of the supersymmetries, in fact at least half of them. (Any set of at least five supersymmetries would include one whose square would generate in the asymptotic region a translation along the first factor of $D_{R} \sim I \times \widetilde{S}^{1}$, but such a translation cannot be extended to a symmetry of $D_{R}$.) There is a standard way [35] to make a topological twist so that half of the asymptotic supersymmetries are preserved in the exact $D_{R}$ geometry. The supersymmetries that are preserved are the ones that are invariant under a rotation of the tangent space of $I \times$ $\widetilde{S}^{1}$ together with an $\mathrm{S} U(2)_{R}$ rotation. The rotation of the tangent space of $I \times \widetilde{S}^{1}$ is generated by $\Gamma_{23}$, and up to conjugation in $\mathrm{S} U(2)_{R}$, we can assume that the $\mathrm{S} U(2)_{R}$ rotation in question is generated by $\sigma_{23}$. So with the standard topological twist, the four supersymmetries that are preserved are the ones that can be characterized, in the asymptotic region of $D_{R}$, by

$$
\left(\Gamma_{23}+\sigma_{23}\right) \eta=0
$$

Let us look at this from the point of view of toroidal compactification, on $S^{1} \times \widetilde{S}^{1}$, to $\Sigma=\mathbb{R} \times I$. The tip of the cigar at $r=0$ gives a boundary condition at one end of $I$. This boundary condition preserves half of the supersymmetry. In other words, in the effective two-dimensional sigma-model, the tip, with the standard topological twist, determines a half-BPS brane $\mathcal{B}_{*}$. We would like to interpret in two-dimensional terms the unbroken supersymmetry and the brane that carries this symmetry. A convenient way to do this is to understand what topological properties this brane possesses. What structures of twisted topological field theory on $\Sigma$ are preserved by this brane?

Any topological field theory structure on $\Sigma$ is associated with a supersymmetry generator $\eta$ that is invariant under a rotation of the tangent space to $\Sigma$ together with an $\mathrm{S} U(2)_{R}$ transformation. The rotation of the tangent space is generated by $\Gamma_{02}$, and as this anticommutes with the matrix $\Gamma_{23}$ that appears on the left of eq. (3.4), we must pick an $\mathrm{S} U(2)_{R}$ generator that anticommutes with $\sigma_{23}$ or we will reach a contradiction. With no essential loss of generality, we can look for a supersymmetry generator that obeys

$$
\left(\Gamma_{02}+\sigma_{31}\right) \eta=0
$$

The two equations (3.4) and (3.5) characterize a two-dimensional space of $\eta$ 's. To determine a particular topological field theory structure on $\Sigma$, we need one more condition restricting to a one-dimensional space of $\eta$ 's. Any condition will do, so there is a $\mathbb{C P}^{1}$ family of topological field theories on $\Sigma$ that are all compatible with the same brane $\mathcal{B}_{*}$.

For one convenient choice, we supplement (3.4) and (3.5) with the additional condition

$$
\left(\Gamma_{01}+\sigma_{23}\right) \eta=0
$$

Although presented here in a non-invariant way, these three conditions combine to something that can be described invariantly. By commuting the operators appearing on the 
left hand sides of the three equations, one learns that a spinor obeying the three equations actually obeys

$$
\begin{aligned}
\left(\Gamma_{i j}+\sigma_{i j}\right) \eta & =0 \\
\left(\Gamma_{0 i}+\sigma_{i+1, i-1}\right) \eta & =0,
\end{aligned}
$$

for $i, j=1,2,3$. By subtracting (3.6) from (3.4), we find that $\left(\Gamma_{01}-\Gamma_{23}\right) \eta=0$, which together with (3.2) implies that

$$
\Gamma_{0123} \eta=-\eta, \quad \Gamma_{45} \eta=-i \eta .
$$

The conditions (3.7) are the standard conditions that characterize the supersymmetry generator of a twisted four-dimensional topological field theory [35] that (in the case of $\mathrm{SU}(2)$ gauge theory without hypermultiplets) is related to Donaldson theory. They can be characterized in group-theoretic terms. Let $\mathrm{S} O(4)$ be the group of rotations of the tangent space to $M$; denote its double cover as $\mathrm{S} U(2)_{l} \times \mathrm{S} U(2)_{r}$. Then the above conditions mean that $\eta$ is invariant under $\mathrm{S} U(2)_{l} \times \mathrm{S} U(2)_{r}^{\prime}$, where $\mathrm{S} U(2)_{r}^{\prime}$ is a diagonal subgroup of $\mathrm{SU}(2)_{r} \times \mathrm{SU}(2)_{R}$.

We can get two more useful choices of supersymmetry parameter by observing that $\Gamma_{1}, \Gamma_{4}$, and $\Gamma_{5}$ all commute with the operators on the left hand sides of our first two conditions (3.4) and (3.5). So these conditions commute with a group $\mathrm{SO}(3)_{145}$ that rotates those three gamma matrices. Making an $\mathrm{SO}(3)_{145}$ transformation that rotates $\Gamma_{1}$ to $\Gamma_{4}$ or $\Gamma_{5}$, we replace (3.6) by

$$
\left(\Gamma_{04}+\sigma_{23}\right) \eta=0
$$

or

$$
\left(\Gamma_{05}+\sigma_{23}\right) \eta=0
$$

\subsubsection{Support of the brane}

Before trying to interpret the supersymmetries in two dimensional terms, let us first determine the support of the brane $\mathcal{B}_{*}$.

In four dimensions, a single vector multiplet contains a complex scalar field $\phi$ or equivalently a pair of real scalars. When we compactify to three dimensions on a circle $S^{1}$, two more scalars come from the gauge field $A$ - one is the holonomy of $A$ around $S^{1}$, and the second is the dual photon. All four scalars combine to a three-dimensional hypermultiplet. The key point here is that to arrive at this hypermultiplet, a duality transformation was needed, converting the photon to a scalar.

The geometry of the hypermultiplets that arise in compactification can be described as follows. Let $\boldsymbol{B}$ be the Coulomb branch of vacua of the four-dimensional gauge theory. It parametrizes a family of abelian varieties. We denote the total space of this family as $\mathcal{M}_{H}$ because in a large class of examples [16], this total space is a moduli space of Higgs bundles on a Riemann surface $C$. After compactification on a circle and dualization of the photons (one in each vector multiplet), $\mathcal{M}_{H}$ becomes endowed with a hyper-Kahler metric, and one gets [34] a low energy description by a sigma-model of maps from three-dimensional spacetime to $\mathcal{M}_{H}$. 
Compactification to two dimensions on $S^{1} \times \widetilde{S}^{1}$ is a little different. In this case, a four-dimensional gauge field leads to two scalars - its holonomies around the two circles - without dualization of any kind. This actually gives a description by linear multiplets rather than hypermultiplets [39]; this description is inconvenient as there is not a powerful theory of nonlinear models built from linear multiplets. A $T$-duality transformation for the scalar fields that arise from holonomies around one circle or the other is useful because it leads to a description by hypermultiplets, and here hyper-Kahler geometry is an effective tool for studying nonlinear models. (For more on this, see the end of this subsection as well as section 3.1.3.)

From a two-dimensional point of view, in compactification on $S^{1} \times \widetilde{S}^{1}$, there is no natural choice of which of the two sets of scalars should be $T$-dualized. A description in which we $T$-dualize one set of scalars differs from a description in which we $T$-dualize the other set of scalars by a combined $T$-duality on both sets of scalars. The combined operation is a $T$-duality on all the scalars that come from gauge fields, so it can be described simply: it is the $T$-duality on the fibers of the fibration $\mathcal{M}_{H} \rightarrow \boldsymbol{B}$. This particular instance of $T$-duality is related to $S$-duality in another description of the same models [36,37], and is the basic geometric Langlands duality [13]. This $T$-duality transforms $\mathcal{M}_{H}$ to an analogous moduli space of Higgs bundles for the Langlands dual gauge group.

Now let us specialize to our problem with $M=\mathbb{R} \times S^{1} \times D_{R} \sim \mathbb{R} \times S^{1} \times I \times \widetilde{S}^{1}$. The symmetry between $S^{1}$ and $\widetilde{S}^{1}$ is broken by the fact that $\widetilde{S}^{1}$, and not $S^{1}$, is capped off at the tip of the cigar. It turns out that to explain the results of [6], it is better to $T$-dualize the holonomies of the gauge field around $\widetilde{S}^{1}$. (In section 4 , we will explore another problem in which the two circles enter symmetrically.)

Recalling that we have labeled the four dimensions of $M \sim \mathbb{R} \times S^{1} \times I \times \widetilde{S}^{1}$ consecutively as 0123 , we write simply $A_{1}$ or $A_{3}$ for scalars arising from the holonomy of a gauge field $A$ around $S^{1}$ or $\widetilde{S}^{1}$, respectively. The boundary conditions on $\phi, A_{1}$, and $A_{3}$ at the tip of the cigar are uniquely determined, since in four-dimensional terms there is no boundary at all. There is no reason for $\phi$ or $A_{1}$ to vanish at the tip of the cigar, so in the two-dimensional description on $\Sigma=\mathbb{R} \times I$, they obey Neumann boundary conditions. On the other hand, $A_{3}$ must vanish because it is the holonomy of the gauge field around a circle that shrinks to a point at the tip. So $A_{3}$ obeys Dirichlet boundary conditions.

However, to get a description by a two-dimensional sigma-model with target $\mathcal{M}_{H}$, we are supposed to $T$-dualize $A_{3}$, replacing it by another scalar that we will call $\varrho$. For future reference, ${ }^{4}$ we write the equations describing this $T$-duality:

$$
\begin{aligned}
& \partial_{0} \varrho=-i \partial_{2} A_{3} \\
& \partial_{2} \varrho=i \partial_{0} A_{3} .
\end{aligned}
$$

After T-duality, $\varrho$ obeys Neumann boundary conditions at the tip of the cigar. In fact, at this stage all scalars $\phi, A_{1}$, and $\varrho$ obey Neumann boundary conditions at the tip. So

\footnotetext{
${ }^{4}$ Here we assume the circles $S^{1}$ and $\widetilde{S}^{1}$ are orthogonal; otherwise $A_{1}$ enters the formulas. See the appendix for a much more complete treatment.
} 
the tip of the cigar corresponds in two-dimensional terms to a brane $\mathcal{B}_{*}$ whose support is all of $\mathcal{M}_{H}$.

The topological twist that was used to preserve supersymmetry on $\mathbb{R} \times S^{1} \times D_{R}$ does not generate at the tip of $D_{R}$ any couplings that look obviously like Chan-Paton couplings. So it is natural to think that the Chan-Paton bundle of $\mathcal{B}_{*}$ may be trivial. If so, as the support of $\mathcal{B}_{*}$ (being all of $\mathcal{M}_{H}$ ) is holomorphic in every complex structure on $\mathcal{M}_{H}, \mathcal{B}_{*}$ will be a brane of type $(B, B, B)-$ a $B$-brane for every complex structure that makes up the hyper-Kahler structure of $\mathcal{M}_{H}$. We will show in section 3.1.2 that this is the case.

We will add a word on the more naive description by scalars $\phi, A_{1}$, and $A_{3}$ without any $T$-duality. In this description, precisely one scalar in each hypermultiplet (namely $A_{3}$ ) obeys Dirichlet boundary conditions. Since the usual supersymmetric branes have Dirichlet boundary conditions for an even number of scalars in each multiplet, this is another indication that a simple description requires $T$-duality for either $A_{1}$ or $A_{3}$. (Without such a $T$-duality, a four-dimensional vector multiplet reduces in two dimensions to a linear multiplet rather than a hypermultiplet.)

\subsubsection{Two-dimensional interpretation of the supersymmetries}

Now we want to determine the two-dimensional interpretation of the supercharges whose generator $\eta$ obeys (3.4) and (3.5) plus one of the three supplementary conditions (3.6), (3.9), or $(3.10)$.

We first consider the case of a spinor $\eta$ obeying (3.6). We claim that $\eta$ generates the topological symmetry of the $B$-model in complex structure $I$ - the complex structure in which the Hitchin fibration is holomorphic.

The basic functions on $\mathcal{M}_{H}$ that are holomorphic in complex structure $I$ are $\phi$ and $A_{1}+i \varrho$. So, if $Q$ is the supersymmetry generated by $\eta$, we must show that $[Q, \phi]=$ $\left[Q, A_{1}+i \varrho\right]=0$.

It is straightforward to show that $[Q, \phi]=0$; this is actually a standard fact in the context of applications to Donaldson theory [35]. As $\phi=\left(A_{4}-i A_{5}\right) / \sqrt{2}$ and $\delta A_{I}=i \bar{\eta} \Gamma_{I} \Psi$, what we need to show is that $\bar{\eta}\left(\Gamma_{4}-i \Gamma_{5}\right) \Psi=0$, which will follow if $\left(\Gamma_{4}-i \Gamma_{5}\right) \eta=0$. This is equivalent to $\Gamma_{45} \eta=-i \eta$, which was deduced in (3.8).

The other condition $\left[Q, A_{1}+i \varrho\right]=0$ is more subtle, because $\varrho$ is defined via a $T$-duality that only makes sense after reduction to two dimensions. So in analyzing this condition, we work in the effective two-dimensional theory. Thus, we discard terms involving derivatives in the 1 or 3 directions, and for example that means that $F_{01}$ reduces to $\partial_{0} A_{1}$. The zero mode of a scalar field such as $\varrho$ that is defined via $T$-duality is subtle to understand. However, there are straightforward formulas (3.11) for the derivatives of $\varrho$. So we will content ourselves with showing the vanishing of the derivatives along $\mathbb{R} \times I$ of $\left[Q, A_{1}+i \varrho\right]$ in the effective two-dimensional theory. For example, the derivative in the 0 direction is $\left[Q, \partial_{0} A_{1}+i \partial_{0} \varrho\right]=\left[Q, \partial_{0} A_{1}+\partial_{2} A_{3}\right]=\left[Q, F_{01}+F_{23}\right]$. This vanishes; indeed, the combination $F_{01}+F_{23}$ is self-dual and therefore is $Q$-exact and in particular $Q$-closed in the topological field theory related to Donaldson theory. The derivative of $\left[Q, A_{1}+i \varrho\right]$ in the 2 direction vanishes similarly. 
What we have learned then is that if we select a spinor $\eta$ using the supplementary condition (3.6), we get the topological supercharge of the $B$-model of $\mathcal{M}_{H}$ in complex structure $I$. This is the complex structure in which the Hitchin fibration $\mathcal{M}_{H} \rightarrow \boldsymbol{B}$ is holomorphic, and the scalar fields that are functions on $\boldsymbol{B}$ are likewise holomorphic.

The other conditions that we want to analyze, namely (3.9) and (3.10), can be formally obtained from (3.6), which we have just analyzed, by an $\mathrm{S} O(3)_{145}$ transformation that exchanges $A_{1}$ with $A_{4}$ or $A_{5}$. This fact can be used to interpret the supersymmetries in the low energy theory without any computation. From each vector multiplet, we get four scalars in the effective two-dimensional sigma-model. Schematically we call them $A_{1}, A_{4}$, $A_{5}$, and $\varrho$. Each set of four scalars forms a hypermultiplet whose tangent space admits an action of the quaternion units $I, J$, and $K$. A formal $\mathrm{S} O(3)_{145}$ rotation that exchanges $A_{1}$ with $A_{4}$ or $A_{5}$, so as to map (3.6) to (3.9) or (3.10), maps $I$ to $J$ or $K$. So while the auxiliary condition (3.6) determines $\eta$ to be the generator of the $B$-model in complex structure $I,(3.9)$ or (3.10) similarly determines $\eta$ to be the generator of the $B$-model in complex structure $J$ or $K$, respectively.

At this level of generality, it is in part a convention which of the complex structures on $\mathcal{M}_{H}$ is called $J$ rather than $K$. In a large class of models [16] in which $\mathcal{M}_{H}$ actually is a moduli space of Higgs bundles on a Riemann surface $C$, we can fix the definition of $A_{4}$ and $A_{5}$ and the conventions in the Higgs bundle equations so that $J$ is the complex structure in which $\mathcal{M}_{H}$ parametrizes flat bundles on $C$ with complex structure group. $K=I J$ is then distinct from $J$ but equivalent to it by a $\mathrm{U}(1)_{R}$ rotation, provided hypermultiplet bare masses (which violate $\mathrm{U}(1)_{R}$ ) are absent. The notation just described is in accord with that of [17].

\subsubsection{A mixed $A B$-model}

Now we can be more precise about what would happen if we describe the two-dimensional effective field theory with the naive set of scalars $\phi, A_{1}$, and $A_{3}$, without any $T$-duality.

For example, let us consider the supercharge $Q$ of Donaldson theory, the one that we associated with the $B$-model of complex structure $I$. Making or not making a $T$-duality on $A_{1}$ or $A_{3}$ does not affect the fact that $\phi$ obeys the $B$-model condition $[Q, \phi]=0$. But in the absence of any $T$-duality, the conditions obeyed by $A_{1}$ and $A_{3}$ are $A$-model conditions, not $B$-model conditions.

Indeed, in our derivation, we used the fact that $\partial_{0} A_{1}+\partial_{2} A_{3}$ is $Q$-exact in the effective two-dimensional theory; similarly, the same is true of $\partial_{0} A_{3}-\partial_{2} A_{1}$. We can combine these statements into the assertion that $\left(\partial_{0}+i \partial_{2}\right)\left(A_{3}+i A_{1}\right)$ is $Q$-exact. In the complex structure on $\mathbb{R} \times I$ in which $z=x^{0}+i x^{2}$ is holomorphic, this says that $A_{3}+i A_{1}$ is holomorphic modulo $\{Q, \cdot\}$.

Thus the model under discussion, in terms of the obvious variables without any $T$ duality, is from the point of view of the supercharge $Q$ a mixed $A B$ model, with $B$-model conditions on $\phi$ and $A$-model conditions on $A_{3}+i A_{1}$. A similar story holds if $Q$ is replaced by one of the other supercharges considered above. 


\subsubsection{More on compactification}

We will conclude this discussion with a few more observations about compactification of $\mathcal{N}=2$ gauge theories from four to two dimensions. The goal is to review some points made in $[36,37]$ and derive some formulas that will be used later. (See the appendix for a much more complete treatment.)

We will here consider only compactification of a $\mathrm{U}(1)$ vector multiplet on a rectangular torus $T^{2}$. We write $\mathcal{e}, \mathcal{C}^{\prime}$ for the circumferences of the two circles. Also, for simplicity, we take the four-dimensional $\theta$-angle to vanish.

The scalar $\phi$ in the vector multiplet reduces to a scalar in two dimensions that we denote as $a$. Its kinetic energy is

$$
\frac{\mathcal{C C}^{\prime}}{e^{2}} \int \mathrm{d}^{2} x|\nabla a|^{2}
$$

The components of the gauge field along $T^{2}$ have zero modes that reduce in two dimensions to angle-valued field $b, b^{\prime}$. Their kinetic energy is

$$
\frac{1}{e^{2}} \int \mathrm{d}^{2} x\left(\frac{\mathrm{C}^{\prime}}{\mathrm{C}}|\nabla b|^{2}+\frac{\mathrm{C}^{\prime}}{\mathrm{C}^{\prime}}\left|\nabla b^{\prime}\right|^{2}\right)
$$

However, we want a description obtained by $T$-duality on one of these scalars. Making a $T$-duality that replaces $b^{\prime}$ by another angle-valued field $\varrho,(3.13)$ is replaced by

$$
\frac{\varrho^{\prime}}{\varrho} \int \mathrm{d}^{2} x\left(\frac{1}{e^{2}}|\nabla b|^{2}+\frac{e^{2}}{16 \pi^{2}}|\nabla \varrho|^{2}\right)
$$

In this simple model, the angles $b, \varrho$ parametrize the fibers of the Hitchin fibration. Clearly, the area of a fiber $\boldsymbol{F}$ of the Hitchin fibration is independent of $e^{2}$ and is proportional to $\mathfrak{C}^{\prime} / \mathcal{C}$. So $\mathfrak{C}^{\prime} / \mathcal{C}$ is a Kahler parameter, as in $[36,37]$. If we rescale $a$ to $\widetilde{a}=a \mathfrak{C} / e$, then the metric on $\mathcal{M}_{H}$ become $\mathfrak{C}^{\prime} / \mathcal{C}$ times a metric that depends only on $e$ and not on $\mathcal{C}$ or $\mathfrak{C}^{\prime}$ :

$$
\mathrm{d} s^{2}=\frac{\mathcal{C}^{\prime}}{\varrho}\left(|\mathrm{d} \widetilde{a}|^{2}+\frac{1}{e^{2}} \mathrm{~d} b^{2}+\frac{e^{2}}{16 \pi^{2}} \mathrm{~d} \varrho^{2}\right) .
$$

Conversely, the complex structure of $\boldsymbol{F}$ is determined by $e^{2}$ and is independent of $\mathcal{C}$ and $\mathcal{C}^{\prime}$. In fact, the $\tau$ parameter of $\boldsymbol{F}$ is that of the underlying gauge theory. If one thinks of the $\mathrm{U}(1)$ vector multiplet as arising from compactification of the abelian version of the six-dimensional $(0,2)$ theory on a two-torus $C$, then $\mathcal{M}_{H}$ is the moduli space of $\mathrm{U}(1)$ Higgs bundles on $C$. This picture extends to more interesting examples with $\mathrm{U}(1)$ replaced by a nonabelian group and $C$ by a more general Riemann surface. The effective two-dimensional description in an example of this type is obtained by compactifying the six-dimensional $(0,2)$ theory to two dimensions on $T^{2} \times C$. If one compactifies first on $C$, one gets a generalized quiver theory [16] in four dimensions in which the complex structure of $C$ is encoded in the gauge coupling parameters (generalizing what we called $e^{2}$ in the abelian theory). If one compactifies first on $T^{2}$, one gets $\mathcal{N}=4$ super Yang-Mills theory with coupling parameter $\tau=i \mathfrak{C}_{1} / \mathfrak{C}_{2}$. Further compactification on $C$ gives the situation 
studied in [13]: supersymmetric vacua correspond to Higgs bundles on $C$, so the moduli space of vacua is $\mathcal{M}_{H}$, and the metric on $\mathcal{M}_{H}$ is scaled by $\operatorname{Im} \tau=\mathfrak{C}_{1} / \mathfrak{C}_{2}$, generalizing the abelian result of (3.15).

Since the metric has a factor of $\mathcal{C}^{\prime} / \mathcal{C}$, the Kahler forms all contain this factor as well. Writing $\left(\omega_{I}^{*}, \omega_{J}^{*}, \omega_{K}^{*}\right)$ for the Kahler forms computed relative to the metric (3.15), these Kahler forms are $\mathcal{C}^{\prime} / \mathcal{C}$ times the standard ones defined in eqs. (2.17), (2.18):

$$
\left(\omega_{I}^{*}, \omega_{J}^{*}, \omega_{K}^{*}\right)=\frac{\mathrm{e}^{\prime}}{\mathfrak{e}}\left(\omega_{I}, \omega_{J}, \omega_{K}\right) .
$$

Of course, this factor will also appear in the holomorphic two-forms. For example, the holomorphic two-form in complex structure $I$ is

$$
\Omega_{I}^{*}=\omega_{J}^{*}+i \omega_{K}^{*}=\frac{\mathfrak{C}^{\prime}}{\mathfrak{C}}\left(\omega_{J}+i \omega_{K}\right) .
$$

In fact, $\Omega_{I}^{*}=\left(e \mathrm{C}^{\prime} / 2 \pi \mathrm{C}\right) \mathrm{d} \widetilde{a} \wedge\left(\mathrm{d} \varrho-\left(4 \pi i / e^{2}\right) \mathrm{d} b\right)=\left(\mathrm{C}^{\prime} / 2 \pi\right) \mathrm{d} a \wedge\left(\mathrm{d} \varrho-\left(4 \pi i / e^{2}\right) \mathrm{d} b\right) . \quad$ It is convenient to introduce $a_{D}=\tau a$, with ${ }^{5} \tau=4 \pi i / e^{2}$, in terms of which

$$
\Omega_{I}^{*}=\frac{\mathrm{C}^{\prime}}{2 \pi}\left(\mathrm{d} a \wedge \mathrm{d} \varrho-\mathrm{d} a_{D} \wedge \mathrm{d} b\right) .
$$

The expression in parentheses is a standard formula for the holomorphic two-form of the total space of the Seiberg-Witten fibration over the Coulomb branch. (This holomorphic two-form is unaffected by compactification.) The main purpose of computing $\Omega_{I}^{*}$ here has been to explain the factor of $\mathfrak{C}^{\prime}$.

The formula (3.18) has an analog for the general case of a semi-simple gauge group of rank $r$. Pick a local description of the Coulomb branch in terms of $r$ vector multiplets with scalar components $a_{i}$ and dual scalar fields $a_{D}^{i}=\partial \mathcal{F} / \partial a_{i}$ (here $\mathcal{F}$ is the prepotential of the gauge theory). The gauge field of the $i^{\text {th }}$ vector field gives two angle-valued fields $\varrho^{i}$ and $b_{i}$ (as in the $\mathrm{U}(1)$ case, $b_{i}$ is a holonomy and $\varrho_{i}$ is the dual of a holonomy). The analog of $(3.18)$ is then

$$
\Omega_{I}^{*}=\frac{\mathrm{e}^{\prime}}{2 \pi} \sum_{i}\left(\mathrm{~d} a_{i} \wedge \mathrm{d} \varrho^{i}-\mathrm{d} a_{D}^{i} \wedge \mathrm{d} b_{i}\right)
$$

Because it requires the choice of a duality frame, this formula is valid only locally on the Coulomb branch. Given the choice of a duality frame, the formula can be derived by the same steps as in the U(1) case, starting with the low energy effective action on the Coulomb branch.

\subsection{Rethinking the $\Omega$-deformation}

By now, we have learned that the brane associated with the tip of the cigar manifold $D_{R}$ is a brane of type $(B, B, B)$ whose support is all of $\mathcal{M}_{H}$. On the other hand, we know from section 2.3 that this is not what we need in order to generate the quantization of a real slice in $\mathcal{M}_{H}$. What we need is a brane of support $\mathcal{M}_{H}$ and of type $(A, B, A)$.

\footnotetext{
${ }^{5}$ Here $\tau$ is imaginary since we took the four-dimensional $\theta$-angle to vanish. For a derivation including the $\theta$-angle, see eqs. (2.34) and (3.16) of [38].
} 
It is also clear from [6] what we need to do in order to get the desired result: we need to implement an $\Omega$-deformation - that is, we need to replace $D_{R}$ by its $\Omega$-deformed version $D_{R, \varepsilon}$, where $\varepsilon$ is the parameter of the $\Omega$-deformation.

\subsubsection{The Standard formulation}

First, let us recall a standard way of introducing the $\Omega$-deformation. One uses the fact that the scalar fields $A_{4}$ and $A_{5}$ can be viewed as components of the gauge field $A_{I}$ in two extra dimensions. For simplicity, let us focus on $A_{4}$. We think of $x^{4}$ as an angular variable. Instead of taking spacetime to be a product $\mathbb{R}^{4} \times S^{1}$, with the second factor parametrized by $x^{4}$, we take it to be an $\mathbb{R}^{4}$ bundle over $S^{1}$, with the monodromy around $S^{1}$ being an element $g \in \mathrm{S} O(4)$. The monodromy action on $\mathbb{R}^{4}$ can be accompanied by an $R$-symmetry transformation $r \in \mathrm{S} U(2)_{R}$ acting on the fermions, and then the unbroken supersymmetries are those that are invariant under the product $g r$. For our present purposes, we take $g$ to rotate a two-plane in $\mathbb{R}^{4}$, so $g$ actually lies in an $\mathrm{S} O(2)$ subgroup of $\mathrm{SO}(4)$. The element $r$ is chosen in the usual way so that the product $g r$ preserves one-half of the supersymmetry.

Now instead of taking the metric on $\mathbb{R}^{4} \times S^{1}$ to be a simple product, we consider a fiber bundle metric in which the monodromy around $S^{1}$ is the element $g r \in \mathrm{S} O(4) \times \mathrm{S} U(2)_{R}$ (in other words, the geometric monodromy is $g$, and we also make an $R$-symmetry twist by $r)$. In formulas, the metric is

$$
\mathrm{d} s^{2}=\sum_{\mu=0}^{3}\left(\mathrm{~d} x^{\mu}-\varepsilon V^{\mu} \mathrm{d} x^{4}\right)^{2}+\left(\mathrm{d} x^{4}\right)^{2},
$$

The $\Omega$-deformed four-dimensional theory is defined by starting with five-dimensional super Yang-Mills theory in this spacetime, and then taking the fields to be independent of $x^{4}$.

The $\Omega$-deformation has its name because it actually is a deformation of the action. A basis of orthornormal vector fields for the metric (3.20) is given by $u_{\mu}=\partial / \partial x^{\mu}, \mu=$ $0, \ldots, 3, u_{4}=\partial / \partial x^{4}+\varepsilon V^{\mu} \partial / \partial x^{\mu}$. The only one that is unusual is $u_{4}$, and the extra term in $u_{4}$ means that the $\varepsilon$-dependence of the Lagrangian can be computed roughly by a substitution $A_{4} \rightarrow A_{4}+\varepsilon V^{\mu} D_{\mu}$. More precisely, the deformation of the bosonic part of the action can be computed by the following substitutions

$$
\begin{aligned}
{\left[A_{4}, A_{5}\right] } & \rightarrow\left[A_{4}, A_{5}\right]+\varepsilon V^{\mu} D_{\mu} A_{5} \\
D_{\nu} A_{4} & \rightarrow D_{\nu} A_{4}+\varepsilon V^{\mu} F_{\nu \mu} .
\end{aligned}
$$

The Yukawa couplings containing $A_{4}$ are modified in a similar way.

The $\Omega$-deformation preserves whatever supersymmetry commutes with $g r$. However, the supersymmetry algebra is modified. The reason for this is that usually the supersymmetry algebra of $\mathcal{N}=2$ super Yang-Mills theory closes modulo gauge transformations generated by $A_{4}$ and $A_{5}$. But in the present situation, $A_{4}$ is effectively replaced by $A_{4}+\varepsilon V^{\mu} D_{\mu}$, and so wherever a gauge transformation generated by $A_{4}$ would usually appear, there is now an additional term that is $\varepsilon$ times the conserved charge $\mathcal{W}_{V}$ associated to the Killing vector field $V^{\mu}$. The most important special case [7] concerns the supercharge $Q$ that is associated to Donaldson theory and the counting of instantons. In the undeformed theory, 
its square is a gauge transformation; after $\Omega$-deformation, it obeys $Q^{2}=\varepsilon \mathcal{W}_{V}$ modulo a gauge transformation.

\subsubsection{An alternative description}

In constructing the $\Omega$-deformation, we can replace $\mathbb{R}^{4} \times S^{1}$ by $M \times S^{1}$, where $M$ is any Riemannian four-manifold and $g$ is an isometry of $M$. Our application will be to the case that $M=\mathbb{R} \times S^{1} \times D_{R}$, with $g$ a rotation of the cigar metric $D_{R}$ that leaves fixed the tip of the cigar.

In its asymptotic region, $D_{R}$ is simply a product $I \times \widetilde{S}^{1}$, with the circumference of $\widetilde{S}^{1}$ being $2 \pi \rho$. Rotations of $D_{R}$ act in the asymptotic region by rotations of $\widetilde{S}^{1}$. Such rotations of the flat metric $\mathbb{R} \times S^{1} \times I \times \widetilde{S}^{1}$ preserve all supersymmetry, and are not accompanied by an $R$-symmetry transformation. ${ }^{6}$

In principle, we could now proceed to study the claim of [6] in the context of the $\Omega$-deformed theory. The only problem is that the $\Omega$-deformed theory is a different theory from $\mathcal{N}=2$ super Yang-Mills theory, and we have much less experience with its dynamics.

Here we will avoid having to understand the dynamics of $\Omega$-deformed theories, via the following device. We will show that if $M$ is of the form $W \times \widetilde{S}^{1}$, with a product metric, then the $\Omega$-deformation associated to a rotation of $\widetilde{S}^{1}$ can be eliminated by a change of variables. Of course, our $M$ is of the stated form.

We proceed as follows. In the product situation, the $\Omega$-deformation can be described formally as a substitution

$$
A_{4} \rightarrow \widetilde{A}_{4}=A_{4}+\varepsilon \rho \frac{D}{D x^{3}}
$$

(The reason for the factor of $\rho$ is that, as $x^{3}$ has period $2 \pi \rho$, the normalized generator of the rotation of $\widetilde{S}^{1}$ is the vector field $\rho \partial / \partial x^{3}$.) This is a substitution only in a very formal sense; because the right hand side of (3.22) is a differential operator of degree 1 rather than a field, this operation does not make sense as a change of variables in any standard sense. However, the substitution (3.22) does make sense as a formal device to generate a deformation of the action of $\mathcal{N}=2$ super Yang-Mills theory. The reason for this is that $A_{4}$ enters the action only via commutators such as $\left[A_{4}, A_{5}\right]$ and covariant derivatives $D_{\nu} A_{4}$. Under the substitution (3.22), these transform in a sensible way as indicated in eq. (3.21).

Now, consider the transformation

$$
\frac{D}{D x^{3}} \rightarrow \frac{D}{D x^{3}}-\varepsilon \rho A_{4}
$$

Though superficially similar, this does make sense as an ordinary change of variables in the quantum field theory, since it is equivalent to

$$
A_{3} \rightarrow \widetilde{A}_{3}=A_{3}-\varepsilon \rho A_{4}
$$

and the right hand is a field rather than a differential operator of positive degree.

\footnotetext{
${ }^{6}$ This may be understood as follows. To preserve half of the supersymmetry on $\mathbb{R} \times S^{1} \times D_{R}$, in the absence of the $\Omega$-deformation, one twists the fermions, changing their spins. This modifies how they transform under rigid rotations of $D_{R}$ in the curved region near the tip of the cigar, but not in the asymptotic region.
} 
Since the distinction between the formal operation (3.22) and an ordinary change of variables such as (3.23) will be important, we will belabor the point slightly. If the ordinary change of variables (3.23) is applied to a given quantum field theory, we simply get an equivalent theory written in terms of a different set of variables. By contrast, the operation (3.22) is not a change of variables in any ordinary sense, and when it is applied to a theory to which it can be applied, it gives a theory that in general is inequivalent. In general, the $\Omega$-deformation really is a non-trivial deformation. However, we will show here that in the special case of a product $W \times \widetilde{S}^{1}$ and a deformation involving a rotation of the second factor, the $\Omega$-deformation is trivial, in the sense that it can be removed by an ordinary change of variables. The identification of the deformed theory with the undeformed one will, however, involve a non-trivial transformation of the observables, and that is why the analysis will lead to something useful.

The combination of (3.22) and (3.23) can be viewed, formally, to first order in $\varepsilon$, as a rotation of the 34 plane. As such, it leaves fixed, to first order, the bosonic part of the Lagrangian that contains $A_{3}$ and $A_{4}$. For example, the contributions

$$
\int_{W \times \widetilde{S}^{1}} \mathrm{~d}^{4} x \sum_{\mu=0,1,2} \operatorname{Tr}\left(F_{\mu 3}^{2}+D_{\mu} A_{4}^{2}\right)
$$

are invariant to first nontrivial order in $\varepsilon$ under the combination of the formal operation (3.22), which we implement via (3.21), and the actual change of variables (3.23). The same is true for the other bosonic terms involving $A_{3}$ and $A_{4}$, namely

$$
\int_{W \times \widetilde{S}^{1}} \mathrm{~d}^{4} x \operatorname{Tr}\left(\left(D_{3} A_{5}\right)^{2}+\left[A_{4}, A_{5}\right]^{2}\right)
$$

and

$$
\int_{W \times \widetilde{S}^{1}} \mathrm{~d}^{4} x \operatorname{Tr}\left(D_{3} A_{4}\right)^{2}
$$

To compensate for the $\Omega$-deformation of the terms in the action involving fermions, we must make a similar rotation of the fermions

$$
\Psi \rightarrow \exp \left(\frac{\varepsilon \rho}{2} \Gamma_{34}\right) \Psi
$$

The formulas we have considered so far compensate for the $\Omega$-deformation only to first non-trivial order in $\varepsilon$, because the formulas (3.22) and (3.23) represent a rotation of the 34 plane only to that order. (By contrast, for the fermions we have used an exact rotation matrix in (3.28).) How can we improve our formulas to represent a rotation of the 34 plane beyond first order?

For $A_{4}$, it is fairly clear what to do. We compose the $\Omega$-deformation with an ordinary change of variables $A_{4} \rightarrow \widehat{A}_{4}=A_{4} / \sqrt{1+\varepsilon^{2} \rho^{2}}$. So the modified $A_{4}$ becomes

$$
\widehat{A}_{4}=\frac{1}{\sqrt{1+\varepsilon^{2} \rho^{2}}}\left(A_{4}+\varepsilon \rho \frac{D}{D x^{3}}\right) \text {. }
$$

Now it is more or less clear what the remaining formula for the redefinition of $A_{3}$ ought to be. To get a rotation of the 34 plane, we would like the formula to be in some sense

$$
\frac{D}{D x^{3}} \rightarrow \frac{1}{\sqrt{1+\varepsilon^{2} \rho^{2}}}\left(\frac{D}{D x_{3}}-\varepsilon \rho A_{4}\right) \text {. }
$$


The only problem is to explain what this formula means, as we are not free to rescale the derivative $\partial / \partial x^{3}$.

We should interpret rescaling of $D / D x^{3}$ as rescaling of this covariant derivative referred to an orthonormal frame, which will result from rescaling of the metric in the $x^{3}$ direction. Instead of saying that $x^{3}$ has period $2 \pi \rho$, we write $x^{3}=\rho w$ where $w$ is an ordinary angular variable of period $2 \pi$. Thus the metric on $\mathbb{R} \times S^{1} \times I \times \widetilde{S}^{1}$ becomes

$$
\mathrm{d} s^{2}=\sum_{\mu=0}^{2}\left(\mathrm{~d} x^{\mu}\right)^{2}+\rho^{2} \mathrm{~d} w^{2} .
$$

Let us now rewrite the contribution (3.25) to the action making explicit the dependence on $g_{w w}=\rho^{2}$ and also on the gauge coupling constant $e^{2}$ :

$$
\frac{1}{e^{2}} \int_{W \times \widetilde{S}^{1}} \mathrm{~d}^{3} x \mathrm{~d} w \sqrt{g_{w w}} \sum_{\mu=0,1,2} \operatorname{Tr}\left(g_{w w}^{-1} F_{\mu w}^{2}+D_{\mu} A_{4}^{2}\right) .
$$

For this to be invariant, we should rotate $A_{4}$ into $g_{w w}^{-1 / 2} D / D w$, which is the covariant derivative referred to an orthonormal frame. This will entail rescaling of $g_{w w}=\rho^{2}$. At the same time we will rescale $e^{2}$ to ensure that

$$
\frac{\sqrt{g_{w w}}}{e^{2}}
$$

remains fixed. Given this, a rotation of the $A_{4}-X$ plane, where $X=g_{w w}^{-1 / 2} D / D w$ (which in the undeformed theory is the same as $\left.D / D x^{3}\right)$, will leave (3.32) invariant. The equations for the rotation should thus be

$$
\begin{aligned}
A_{4} & =\frac{1}{\sqrt{1+\varepsilon^{2} \rho^{2}}}\left(\widehat{A}_{4}-\varepsilon \rho \widehat{X}\right) \\
X & =\frac{1}{\sqrt{1+\varepsilon^{2} \rho^{2}}}\left(\widehat{X}+\varepsilon \rho \widehat{A}_{4}\right),
\end{aligned}
$$

where it is convenient to express the objects $A_{4}, X$ of the undeformed description in terms of corresponding deformed objects $\widehat{A}_{4}, \widehat{X}$. Interpreting $\widehat{X}$ as $\widehat{g}_{w w}^{-1 / 2} D / D w$, we require

$$
\frac{1}{\sqrt{g_{w w}}}=\frac{1}{\sqrt{1+\varepsilon^{2} \rho^{2}}} \frac{1}{\sqrt{\widehat{g}_{w w}}}
$$

or more simply

$$
\widehat{g}_{w w}=\frac{g_{w w}}{1+\varepsilon^{2} \rho^{2}}=\frac{\rho^{2}}{1+\varepsilon^{2} \rho^{2}} .
$$

To keep (3.33) fixed, we also need to change the gauge coupling so that $\sqrt{\widehat{g}_{w w}} / \widehat{e}^{2}=$ $\sqrt{g_{w w}} / e^{2}$, or

$$
\widehat{e}^{2}=\frac{e^{2}}{\sqrt{1+\varepsilon^{2} \rho^{2}}} .
$$

The parameter $\varepsilon$ is a physical parameter of the $\Omega$-deformation, and for example it enters the results of [6] that we aim to understand. However, the asymptotic radius $\rho$ in 
the $\Omega$-deformed description is not an important parameter for the topological field theory observables of interest.

Accordingly, we could proceed with any choice of $\rho$. However, it turns out that we get a particularly simple description if we take $\rho$ to be very large, $\rho \gg 1 / \varepsilon$, so we will consider this case first. (In fact, in discussions of the $\Omega$-deformation, a flat metric on $\mathbb{R}^{2}$ is typically assumed, rather than our cigar metric $D$. This corresponds to taking $\rho=\infty$.)

The above formulas simplify in the limit $\rho \rightarrow \infty$. The formulas (3.34) expressing the objects $X, A_{4}$ of the $\Omega$-deformed description in terms of analogous objects $\widehat{X}, \widehat{A}_{4}$ in an undeformed description reduce to

$$
\begin{aligned}
A_{4} & =-\widehat{X} \\
X & =\widehat{A}_{4} .
\end{aligned}
$$

Thus, the transformation from the $\Omega$-deformed description to an equivalent undeformed description is a simple $\pi / 2$ rotation of the 34 plane. The limiting value of $\widehat{g}_{w w}$ is $1 / \varepsilon^{2}$, which means that in undeformed language, we take the radius of $\widetilde{S}^{1}$ to be $1 / \varepsilon$ :

$$
\widehat{\rho}=\frac{1}{\varepsilon} .
$$

Finally, for $\rho \rightarrow \infty$, we have $\widehat{e} \rightarrow 0$ and the theory becomes weakly coupled. Since we can make the coupling arbitrarily weak by taking $\rho$ large, one may wonder how the theory can do anything interesting at all. The answer to this question is that our formulas such as (3.37) relating the $\Omega$-deformed theory to an ordinary one are only valid away from the tip of the cigar. For $\rho \rightarrow \infty$, quantum effects remain, but they are localized near the tip of the cigar. Though our explanation here is different in detail, our conclusion is the same as in [7]: when the $\Omega$-deformation is defined using a vector field $V$, quantum effects are localized near zeroes of $V$, because the effective gauge coupling becomes small far away from the zeroes.

Thus, as long as we consider quantities that do not depend on $\rho$, so that we can take $\rho$ large, the $\Omega$-deformed theory on $\mathbb{R} \times S^{1} \times D_{R}$ is equivalent, away from the tip of $D_{R}$, to a weakly coupled and undeformed theory in which the asymptotic radius of $\widetilde{S}^{1}$ is $1 / \varepsilon$. However, in going from the $\Omega$-deformed description to this weakly coupled and undeformed description, physical observables undergo a $\pi / 2$ rotation in the tangent space to the 34 plane. Such a rotation acts on fermions as multiplication by $\left(1+\Gamma_{34}\right) / \sqrt{2}$, so that is the transformation of the supersymmetry generator $\eta$ in comparing the deformed description to the weakly coupled and undeformed one:

$$
\eta \rightarrow \frac{1+\Gamma_{34}}{\sqrt{2}} \eta
$$

Conjugation by this matrix maps $\Gamma_{3}$ to $\Gamma_{4}$ and $\Gamma_{4}$ to $-\Gamma_{3}$.

\subsection{The deformed brane}

We aim to generalize our result of section 3.1 and describe in two-dimensional terms the half-BPS brane $\mathcal{B}_{\varepsilon}$ that arises by compactification on $D_{R, \varepsilon}$, with the $\Omega$-deformation. In $\Omega$ deformed language, the supersymmetries preserved by this brane are those whose generator 
obeys eq. (3.4):

$$
\left(\Gamma_{23}+\sigma_{23}\right) \eta=0 .
$$

Since the symmetry that we used in making the $\Omega$-deformation commutes with the matrix on the left hand side of (3.41), the same condition also characterizes the unbroken supersymmetries of the $\Omega$-deformed brane. However, to describe the $\Omega$-deformed brane in conventional language (in the simplest case, the limit of large $\rho$ ), we must make a rotation of the 34 plane. The rotation angle $\vartheta$ can be read off from eq. (3.34):

$$
\cos \vartheta=\frac{1}{\sqrt{1+\varepsilon^{2} \rho^{2}}}
$$

\subsubsection{The case $\varepsilon \rho \rightarrow \infty$}

Neither the $\Omega$-deformation nor the change of variables that maps us back to the undeformed theory affects the support of the brane $\mathcal{B}_{\varepsilon}$ that comes from the tip of the cigar. So $\mathcal{B}_{\varepsilon}$ is a half-BPS brane whose support is all of $\mathcal{M}_{H}$, just like the brane $\mathcal{B}_{*}$ that arises in the absence of the $\Omega$-deformation. However, the supersymmetry preserved by $\mathcal{B}_{\varepsilon}$ is different from that preserved by $\mathcal{B}_{*}$.

We first consider the simplest case, namely the limit of large $\rho$. In this limit, $\vartheta=\pi / 2$ and the necessary rotation of supersymmetry generators in going from $\Omega$-deformed variables to standard ones was given in eq. (3.40). The rotation in question just transforms $\Gamma_{3}$ to $\Gamma_{4}$, so in a formalism in which the Lagrangian away from the tip of the cigar is standard, the supersymmetries preserved by the $\Omega$-deformed brane $\mathcal{B}_{\varepsilon}$ are characterized not by (3.41) but by

$$
\left(\Gamma_{24}+\sigma_{23}\right) \eta=0 .
$$

Just as in section 3.1, it is convenient to view $M=\mathbb{R} \times S^{1} \times D_{R, \varepsilon}$ as an asymptotic $T^{2}$ bundle over $\mathbb{R} \times I$. We want to understand what topological field theory structures $\mathcal{B}_{\varepsilon}$ preserves in the effective two-dimensional theory.

As in section 3.1, any two-dimensional topological field theory structure is associated to a supersymmetry generator $\eta$ that (modulo a possible $\mathrm{S} U(2)_{R}$ transformation) obeys

$$
\left(\Gamma_{02}+\sigma_{31}\right) \eta=0
$$

The conditions (3.43) and (3.44) select a two-dimensional space of $\eta$ 's. Any non-zero $\eta$ in this two-dimensional space determines a topological field theory, so just like $\mathcal{B}_{*}, \mathcal{B}_{\varepsilon}$ is compatible with a family of topological field theories parametrized by $\mathbb{C P}^{1}$. To select a particular member of this family, we need to place an additional condition on $\eta$; any condition will do.

By analogy with section 3.1, we consider three simple conditions. One of these will be

$$
\left(\Gamma_{23}+\sigma_{12}\right) \eta=0
$$

What we get with this choice is easily determined. The three conditions (3.43), (3.44), and (3.45) are equivalent modulo an $\mathrm{SU}(2)_{R}$ symmetry to the three conditions used in section 3.1 to characterize the $B$-model in complex structure $J$, namely eqs. (3.4), (3.5), 
and (3.9). (To compare our current set of three conditions to the previous three, one uses the fact that if $A \eta=B \eta=0$, then $[A, B] \eta=0$, and one also makes an $\mathrm{S} U(2)_{R}$ transformation $\left(\sigma_{1}, \sigma_{2}, \sigma_{3}\right) \rightarrow\left(\sigma_{3}, \sigma_{2},-\sigma_{1}\right)$.) So the brane $\mathcal{B}_{\varepsilon}$ is a $B$-brane in complex structure $J$.

That is the only complex structure in which $\mathcal{B}_{\varepsilon}$ is a $B$-brane. It turns out that the other simple properties of $\mathcal{B}_{\varepsilon}$ are conveniently understood by imposing on $\eta$ the additional condition

$$
\left(\Gamma_{21}+\sigma_{12}\right) \eta=0
$$

or

$$
\left(\Gamma_{25}+\sigma_{12}\right) \eta=0
$$

together with (3.43) and (3.44):

$$
\begin{aligned}
& \left(\Gamma_{24}+\sigma_{23}\right) \eta=0 \\
& \left(\Gamma_{02}+\sigma_{31}\right) \eta=0 .
\end{aligned}
$$

Either condition (3.46) or (3.47) leads to an $A$-model for the effective topological field theory on $\mathbb{R} \times I$. For example, let us consider (3.47). We will show that it leads to an $A$-model for the symplectic structure $\omega_{I}$. This means that the fields $A_{4}-i A_{5}$ and $A_{1}+i \varrho$ that are holomorphic in complex structure $I$ on $\mathcal{M}_{H}$ will both obey $A$-model conditions; in other words, they depend holomorphically on $z=x^{0}+i x^{2}$, modulo exact terms. We will show this by repeating the analysis of section 3.1.2, but now, instead of the $T$-duality $A_{3} \leftrightarrow \varrho$ converting a mixed $A B$-model to a $B$-model, it will convert a slightly different mixed $A B$-model to an $A$-model.

If one places all $\sigma$-matrices on the right in equations (3.47) and (3.48), and then multiplies the left and right hand sides of the three equations, one learns that

$$
\Gamma_{0245} \eta=-\eta
$$

as a result of which the six-dimensional chirality condition implies also that

$$
\Gamma_{13} \eta=i \eta
$$

Now, consider the group $\mathrm{SO}(4)_{0245}$ that rotates the tangent spaces to the bosonic fields $A_{0}, A_{2}, A_{4}, A_{5}$. Decomposing its double cover as $\mathrm{S} U(2)_{\ell^{\prime}} \times \mathrm{S} U(2)_{r^{\prime}},(3.49)$ implies that $\eta$ is invariant under $\mathrm{S} U(2)_{\ell^{\prime}}$, and eqs. (3.47) and (3.48) further imply that $\eta$ is invariant under a diagonal subgroup of $\mathrm{S} U(2)_{r^{\prime}} \times \mathrm{S} U(2)_{R}$.

In short, $\eta$ is characterized by precisely the conditions that characterize the supersymmetry generator of Donaldson theory, modulo a rotation of the tangent space that exchanges $A_{3}$ with $A_{4}$ and $A_{1}$ with $A_{5}$. This means that we can borrow the analysis of section 3.1.2, except that the roles of $A_{1}+i A_{3}$ and $A_{4}-i A_{5}$ are now reversed. In section 3.1.2, $A_{4}-i A_{5}$ obeyed a $B$-model condition, and $A_{1}+i A_{3}$ obeyed an $A$-model condition that was converted to a $B$-model condition by the $T$-duality $A_{3} \leftrightarrow \varrho$. So now, $A_{4}-i A_{5}$ obeys an $A$-model condition, and $A_{1}+i A_{3}$ obeys a $B$-model condition that is converted to an $A$-model condition by the $T$-duality on $A_{3}$. 
We can analyze the consequence of eq. (3.46) in the same way. This equation differs from (3.47) by the exchange $A_{1} \leftrightarrow A_{5}$. So now, mimicking the last sentence of the last paragraph, $A_{4}-i A_{1}$ obeys an $A$-model condition, and $A_{5}+i A_{3}$ obeys a $B$-model condition that is converted to an $A$-model condition by the $T$-duality on $A_{3}$. So we get an $A$-model in which $A_{4}-i A_{1}$ and $A_{5}+i \varrho$ are holomorphic modulo exact terms.

Given our conventions for what is $J$ and what is $K$, the functions $A_{4}-i A_{1}$ and $A_{5}+i \varrho$ are holomorphic in complex structure $K$, and this means that the $A$-model that we have just arrived at is the $A$-model of symplectic form $\omega_{K}$.

In summary, the brane $\mathcal{B}_{\varepsilon}$ is - in the limit of large $\rho \varepsilon$ - a brane of type $(A, B, A)$. For understanding the results of [6], the most important property is that it is an $A$-brane of type $\omega_{K}$. Indeed, as we see if we rotate back to the deformed language, the topological supercharge of the $A$-model of type $\omega_{K}$ is the one that corresponds to the usual supercharge of the $\Omega$-deformed theory (or equivalently of Donaldson theory). So this is the supercharge that we must use to make contact with the results of [6]. It is also the right one for a different reason described in section 2.3: the coisotropic brane with Chan-Paton curvature $F=\omega_{J}$ in the $A$-model with symplectic structure $\omega_{K}$ is the right tool if we want to study quantization of a real section of the integrable system $\mathcal{M}_{H}$.

By contrast, in section 4, where we make contact with the relation [15] of fourdimensional gauge theory to two-dimensional Liouville theory, the important fact will be that the same brane $\mathcal{B}_{\varepsilon}$ is an $A$-brane for the symplectic structure $\omega_{I}$.

\subsubsection{The general case}

We have by now interpreted the supersymmetry preserved by the tip of the cigar in the $\Omega$-deformed theory in undeformed language for $\varepsilon \rho \gg 1$. However, since the $\Omega$-deformation is most directly understood as a deformation of the standard theory, with $\varepsilon$ as a small deformation parameter, one would naturally like to analyze the opposite limit $\varepsilon \rho \ll 1$. In fact, it is not hard to analyze the general case using tools described in section 2.4.

We recall that a general topological twist in two-dimensions is determined by a pair of complex structures $\left(\mathcal{J}_{+}, \mathcal{J}_{-}\right) \in \mathbb{C P}_{+}^{1} \times \mathbb{C P}_{-}^{1}$. A brane of type $(B, B, B)$ is characterized by $\mathcal{J}_{-}=\mathcal{J}_{+}$. On the other hand, as explained in section 2.4.1, a brane of type $(A, B, A)$ is characterized by $\mathcal{J}_{-}=h \mathcal{J}_{+}$, where $h$ is a $\pi$ rotation around the $J$ axis.

We get from one to the other using the rotation (3.38). We view this rotation as an element $g$ of a group $\mathrm{SO}(4)_{1345}$ that acts on the tangent space to dimensions 1345 . As such, $g$ is a $\pi / 2$ rotation of the 34 plane. The double cover of $\mathrm{SO}(4)_{1345}$ is the group $\Gamma=\mathrm{S} U(2)_{+} \times \mathrm{S} U(2)_{-}$, introduced in section 2.4 .3 , that acts on $\mathbb{C P}_{+}^{1} \times \mathbb{C P}_{-}^{1}$. As a $\pi / 2$ rotation of one plane, $g$ corresponds to a pair $\left(g_{+}, g_{-}\right) \in \mathrm{S} U(2)_{+} \times \mathrm{S} U(2)_{-}$, where each of $g_{ \pm}$corresponds to a $\pi / 2$ rotation in $\mathrm{SO}(3)_{ \pm}=\mathrm{S} U(2)_{ \pm} / \mathbb{Z}_{2}$. The $\mathrm{S} U(2)$ element $h$ of the last paragraph is then $h=g_{-}^{-1} g_{+}$, as noted in section 2.4.3. Given that $h$ is a $\pi$ rotation around the $J$ axis, evidently $g_{+}$and $g_{-}$are rotations around the $J$ axis by respective angles $\pi / 2$ and $-\pi / 2$.

Next let us consider the case of general $\varepsilon \rho . g$ is now a rotation of the 34 plane by a more general angle $\vartheta$. So $g_{+}$and $g_{-}$are rotations around the $J$ axis by angles $\vartheta$ and $-\vartheta$, respectively. 
Now, starting with the fact that in the undeformed theory the tip of the cigar preserves supersymmetry of type $(B, B, B)$, we can understand the supersymmetry preserved by the brane $\mathcal{B}_{\varepsilon}$ for general $\varepsilon \rho$. First of all, complex structure $J$ is invariant under rotations around the $J$ axis. So $\mathcal{B}_{\varepsilon}$ is a $B$-brane of type $J$ for all $\varepsilon \rho$. One can verify this explicitly by observing that the conditions (3.45) and (3.48) are invariant under a rotation of the 34 plane, modulo an $\mathrm{S} U(2)_{R}$ transformation generated by $\sigma_{31}$.

More pressing for our application is to interpret in undeformed variables the usual supercharge of the $\Omega$-deformed theory, which in $\Omega$-deformed variables corresponds to $\mathcal{J}_{+}=$ $\mathcal{J}_{-}=I$. After rotating $\mathcal{J}_{+}$by an angle $\vartheta$ and $\mathcal{J}_{-}$by an angle $-\vartheta$ in the $I K$ plane, they map to

$$
\begin{aligned}
& \mathcal{J}_{+}=I \cos \vartheta+K \sin \vartheta \\
& \mathcal{J}_{-}=I \cos \vartheta-K \sin \vartheta .
\end{aligned}
$$

According to (2.26), the topological field theory with this pair $\left(\mathcal{J}_{+}, \mathcal{J}_{-}\right)$is equivalent to an $A$-model with

$$
\begin{aligned}
\omega^{-1} & =\frac{1}{2}\left(\omega_{+}^{-1}-\omega_{-}^{-1}\right) \\
\omega^{-1} B & =\frac{1}{2}\left(\mathcal{J}_{+}+\mathcal{J}_{-}\right) .
\end{aligned}
$$

Here, writing $g$ for the hyper-Kahler metric on $\mathcal{M}_{H}$, we have $\omega_{ \pm}=g \mathcal{J}_{ \pm}$.

To make (3.52) more explicit, we use $\omega_{+}=\omega_{I}^{*} \cos \vartheta+\omega_{K}^{*} \sin \vartheta, \omega_{-}=\omega_{I}^{*} \cos \vartheta-\omega_{K}^{*} \sin \vartheta$. (The meaning of the asterisks was explained in eq. (3.16): $\omega_{I}^{*}$ and $\omega_{K}^{*}$ are the Kahler forms for the actual Kahler metric on $\mathcal{M}_{H}$, which depends on the radii of the compactification.) Evaluating (2.26), we find that the symplectic form of the equivalent $A$-model is

$$
\omega=\frac{\omega_{K}^{*}}{\sin \vartheta}
$$

So the supersymmetry is that of the $A$-model with symplectic structure a multiple of $\omega_{K}$. The multiple is inessential as explained in the discussion of eq. (2.19). The result agrees, as expected, with what we found for large $\varepsilon \rho$ in section 3.3 .

Eq. (2.26) also tells us how to determine the $B$-field:

$$
\omega^{-1} B=I \cos \vartheta
$$

which implies that $B$ is a multiple of $\omega_{J}$.

\subsubsection{A canonical coisotropic brane}

Since $\omega_{J}$ is cohomologically trivial in a large class of models, as explained in section 2.3.2, one may wonder what we actually learn by determining $B$. The answer is that we do not learn much until we also consider the couplings generated at the tip of the cigar. Those couplings generate a Chan-Paton curvature $F$ for the brane in the effective two-dimensional description, and only the combination $F+B$ is invariant under $B$-field gauge transformations. 
In this paper, we will not attempt to compute the couplings at the tip of the cigar. But we can make a simple observation. Let us consider the limit in which $\varepsilon \rho$ is very small. We approach this limit by keeping $\rho$ fixed and taking $\varepsilon$ to zero. In this limit, the $\varepsilon$-dependent corrections at the tip of the cigar are negligible, and $F$ vanishes. Since also $\vartheta \rightarrow 0$ for $\varepsilon \rho \rightarrow 0$, eq. (3.54) becomes $\omega^{-1}(F+B)=I$.

In other words, for $\varepsilon \rho$ very small, the brane derived from the tip of the cigar approaches the coisotropic brane of type $(A, B, A)$ in which the complex structure $\mathcal{I}=\omega^{-1}(F+B)$ is equal to $I$. Since $\omega=\omega_{K}^{*} / \sin \vartheta$, this brane has $F+B=\omega_{J}^{*} / \sin \vartheta$. It is convenient to evaluate this using (3.19), using the fact that $\omega_{J}^{*}=\operatorname{Re} \Omega_{I}^{*}$, and identifying $\mathrm{C}^{\prime}$ as $2 \pi \widehat{\rho}$ (which for small $\varepsilon \rho$ is the same as $2 \pi \rho)$. For small $\varepsilon \rho$, we can replace $\sin \vartheta$ by $\varepsilon \rho$, whereupon $\rho$ cancels out and we get

$$
F+B=\operatorname{Re} \frac{1}{\varepsilon} \sum_{i}\left(\mathrm{~d} a_{i} \wedge \mathrm{d} \varrho^{i}-\mathrm{d} a_{D}^{i} \wedge \mathrm{d} b_{i}\right)
$$

along with

$$
\omega=\operatorname{Im} \frac{1}{\varepsilon} \sum_{i}\left(\mathrm{~d} a_{i} \wedge \mathrm{d} \varrho^{i}-\mathrm{d} a_{D}^{i} \wedge \mathrm{d} b_{i}\right)
$$

Recalling the formula (3.18) for $\Omega_{I}=\omega_{J}+i \omega_{K}$, it follows that at least for small $\varepsilon$, the brane coming from the tip of the cigar is a space-filling brane in which the complex structure $\mathcal{I}=\omega^{-1}(F+B)$ is equal to $\omega_{K}^{-1} \omega_{J}=I$. Since $\varepsilon \rho$ is small in this computation, the difference between the gauge couplings $e$ and $\widehat{e}$ in $\Omega$-deformed and undeformed variables is not important and $I$ is simply the complex structure in which the Hitchin fibration of the moduli space $\mathcal{M}_{H}$ of vacua is holomorphic.

In the case of a generalized quiver theory [16] that arises by compactification from six dimensions on a Riemann surface $C$, the brane is the canonical coisotropic brane of type $(A, B, A)$ that is naturally defined using the complex structure on $C$ that prevails at the tip of the cigar - the one that is used in the $\Omega$-deformed description. This is the right brane for any $\rho$, since $\rho$ is irrelevant in the twisted topological field theory.

Changing $\rho$ changes $\widehat{e}$ and therefore changes the effective complex structure on $C$. However, the brane that we have found makes sense for any choice of the complex structure on $C$, because the concept of a brane of type $(A, B, A)$ is independent of that complex structure. This was explained in section 2.3.2.

Our determination that the complex structure $\mathcal{I}=\omega^{-1}(F+B)$ is equal to $I$ can be tested in the following way. Let $Q_{\varepsilon}$ be the topological supercharge of the $\Omega$-deformed theory. For $\varepsilon=0$, away from the tip of the cigar, there are no non-trivial $Q_{\varepsilon}$-invariant local operators. But at the tip of the cigar, $Q_{\varepsilon}$ reduces to the topological supercharge $Q$ of Donaldson theory, and its cohomology in the space of gauge-invariant local operators is generated by the gauge-invariant polynomials $\operatorname{Tr} \phi^{n}$ in the scalar field $\phi$; these are the usual local operators in Donaldson theory. In the $\sigma$-model with target $\mathcal{M}_{H}$, the gauge-invariant polynomials in $\phi$ become the holomorphic functions on the base of the Hitchin fibration (and thus, the commuting Hamiltonians of the integrable system). Let us compare this to the answer in the $A$-model with symplectic structure $\omega$. In bulk, the $A$-model admits no 
local observables of ghost number zero; however, on a boundary labeled by a space-filling coisotropic brane with complex structure $\mathcal{I}$, the observables are the holomorphic functions in that complex structure. Thus, the complex structure $\mathcal{I}$ on $\mathcal{M}_{H}$ must be one in which the Hitchin fibration $\pi: \mathcal{M}_{H} \rightarrow \boldsymbol{B}$ is holomorphic — so that the holomorphic functions on $\boldsymbol{B}$ pull back to holomorphic functions on $\mathcal{M}_{H}$. Our result $\mathcal{I}=I$ is consistent with this, since $I$ has the required property.

For this reason and because the deformation theory of coisotropic branes is rather rigid, we believe that the result $\mathcal{I}=I$ or equivalently the determination (3.55) of $F+B$ is exact, even though our derivation was only valid for small $\varepsilon$.

\subsection{Boundary conditions at the far end}

Hitherto, in the effective field theory on $\mathbb{R} \times I$, we have analyzed a distinguished brane $\mathcal{B}_{\varepsilon}$ that arises from the tip of the cigar. It is a space-filling coisotropic brane in an $A$ model with target $\mathcal{M}_{H}$. The symplectic form of the $A$-model is a multiple of $\omega_{K}$, and the gauge-invariant field $F+B$ of the brane is the corresponding multiple of $\omega_{J}$.

By contrast, what happens at the other end of the cigar is largely up to us. We can pick any $A$-model boundary condition we want at the second boundary $r=R$ of $\mathbb{R} \times S^{1} \times D_{R}$. If the aim is to study quantization of a real section of $\mathcal{M}_{H}$, we should pick a boundary condition that determines a Lagrangian $A$-brane with support $L \subset \mathcal{M}_{H}$. Moreover, while Lagrangian for $\omega_{K}, L$ should be symplectic for $\omega_{J}$. We pick a flat line bundle $\mathcal{S}$ over $L$, and write $\mathcal{B}_{L}$ for the corresponding $A$-brane. The space of $\left(\mathcal{B}_{\varepsilon}, \mathcal{B}_{L}\right)$ strings will be a quantization of $L$ with symplectic form $F+B$.

To orient ourselves, we will begin by constructing some choices of $L$ directly by hand, before describing them in four-dimensional gauge theory language. Also, we will begin with the free $\mathrm{U}(1)$ vector multiplet, previously considered in section 3.1.4.

We will consider two simple choices of Lagrangian submanifold. Since the symplectic form in the abelian case is

$$
\omega=\operatorname{Im} \frac{1}{\varepsilon}\left(\mathrm{d} a \wedge \mathrm{d} \varrho-\mathrm{d} a_{D} \wedge \mathrm{d} b\right)
$$

we can define a Lagrangian submanifold $L_{1}$ by $^{7}$

$$
\varrho=0=\operatorname{Im} a_{D} .
$$

And we can define a second Lagrangian submanifold $L_{2}$ by

$$
b=0=\operatorname{Im} a .
$$

Restricted to $L_{1}$, we have $F+B=\mathrm{d} \lambda$ with

$$
\lambda=-\frac{\operatorname{Re} a_{D}}{\varepsilon} \wedge \mathrm{d} b
$$

\footnotetext{
${ }^{7}$ Throughout our derivation, we have taken $\varepsilon$ to be real. However, a generalization to complex $\varepsilon$ may be trivially made by a $\mathrm{U}(1)_{R}$ rotation (which may also act on bare masses and scale parameters of the gauge theory). In this generalization, eq. (3.57) is still valid, and the second conditions in (3.58) and (3.59) should read $\operatorname{Im}\left(a_{D} / \varepsilon\right)=0$ and $\operatorname{Im}(a / \varepsilon)=0$.
} 
In the WKB approximation, quantum states correspond to values of $\operatorname{Re} a_{D}$ for which $\oint \lambda$ is an integer multiple of $2 \pi$. Since $b$ is an angular variable, this condition says that $\operatorname{Re} a_{D} / \varepsilon$ should be an integer. We also set $\operatorname{Im} a_{D} / \varepsilon$ to zero as part of the definition of $L_{1}$. The two conditions combine to

$$
\frac{a_{D}}{\varepsilon} \in \mathbb{Z}
$$

or equivalently

$$
\exp \left(2 \pi i a_{D} / \varepsilon\right)=1 .
$$

Both the result and also the fact that the real and imaginary parts of $a_{D}$ have gone their separate ways in the derivation are hopefully reminiscent of section 2.1. In the case of the free abelian vector multiplet, the WKB approximation is valid.

We can quantize $L_{2}$ similarly. In this case, $F+B=\mathrm{d} \lambda$ with

$$
\lambda=\frac{\operatorname{Re} a}{\varepsilon} \wedge \mathrm{d} \varrho .
$$

Quantization gives

$$
\frac{a}{\varepsilon} \in \mathbb{Z}
$$

or equivalently

$$
\exp (2 \pi i a / \varepsilon)=1 \text {. }
$$

How can we generalize this to the case of a non-abelian gauge group? Given a choice of duality frame, the symplectic form is the obvious generalization of (3.57),

$$
\omega=\operatorname{Im} \frac{1}{\varepsilon} \sum_{i}\left(\mathrm{~d} a_{i} \wedge \mathrm{d} \varrho^{i}-\mathrm{d} a_{D}^{i} \wedge \mathrm{d} b_{i}\right) .
$$

So it looks like we can define an analog of $L_{1}$ by setting $\varrho^{i}=0=\operatorname{Im}\left(a_{D}^{i} / \varepsilon\right)$. Similarly we can imitate the definition of $L_{2}$. The only problem with these definitions is that they only make sense locally, since they require a choice of duality frame.

To find a definition that makes sense globally in the nonabelian case, we need a better point of view. Let us first return to the $\mathrm{U}(1)$ problem and express the definitions of $L_{1}$ and $L_{2}$ in a different way. For gauge group U(1), the moduli space of vacua is the moduli space $\mathcal{M}_{H}$ of $\mathrm{U}(1)$ Higgs bundles on a Riemann surface $T$ that is simply a two-torus parametrized by the angles $\varrho$ and $b$. In complex structure $J, \mathcal{M}_{H}$ parametrizes flat $\mathbb{C}^{*}$ bundles over $T\left(\mathbb{C}^{*}\right.$ is the complexification of $\left.\mathrm{U}(1)\right)$. Such a flat bundle is labeled by its holonomies. Choosing an $A$-cycle and a $B$-cycle that correspond to the $\varrho$ and $b$ directions on $T$, the two holonomies are $U=\exp \left(i\left(\varrho+i \operatorname{Im} a_{D} / \varepsilon\right)\right)$ and $V=\exp (i(b+i \operatorname{Im} a / \varepsilon))$. Thus, our two Lagrangian submanifolds $L_{1}$ and $L_{2}$ can be described by the conditions $U=1$ and $V=1$, respectively.

This definition can be adapted to the nonabelian case, if one considers generalized quiver theories [16] which arise by compactifying from six dimensions on a Riemann surface $C$. In fact, there are multiple ways to do this.

One approach, assuming that $C$ has genus $g$ (and for brevity no punctures), is to pick a set of $g A$-cycles and $g B$-cycles on $C$, generating the first homology of $C$. Then 
one can define a complex Lagrangian submanifold $L \subset \mathcal{M}_{H}$ for complex structure $J$ by requiring the holonomies of the flat bundle around all $A$-cycles (or around all $B$-cycles) to be trivial. This can lead to a construction in which formulas like (3.62) and (3.65) are valid at least asymptotically.

For $G=\mathrm{S} U(2)$, so that $G_{\mathbb{C}}=\mathrm{S} L(2, \mathbb{C})$, another construction of a complex Lagrangian submanifold that in some sense generalizes what we did for $\mathrm{U}(1)$ is as follows. In relation to generalized quiver gauge theories [16], it is convenient to build $C$ from $3 g-3$ cylinders or tubes that are glued together via trinions or "pairs of pants." Topologically, such a construction gives us $3 g-3$ circles, one for each cylinder. A complex Lagrangian submanifold $L \subset \mathcal{M}_{H}$ can be defined by requiring that the holonomy of the flat $\mathrm{S} L(2, \mathbb{C})$ bundle around each circle is unipotent, in other words conjugate to a triangular matrix

$$
\left(\begin{array}{ll}
1 & \star \\
0 & 1
\end{array}\right) \text {. }
$$

A generalization of this Lagrangian submanifold for $G$ of higher rank is not immediately apparent.

To learn more, we now consider the construction of branes on $\mathcal{M}_{H}$ of type $(A, B, A)$ from the point of view of four-dimensional gauge theory.

\subsubsection{Gauge theory realization}

To construct branes of type $(A, B, A)$ in gauge theory, we need half-BPS boundary conditions that preserve those supersymmetries whose generator $\eta$ obeys

$$
M \eta=\eta
$$

or equivalently

$$
\bar{\eta} M=\bar{\eta},
$$

where

$$
M=\Gamma_{24} \sigma_{23} .
$$

This choice of $M$ corresponds to the case $\varepsilon \rho \rightarrow \infty$, so in this analysis we are restricted to that case.

We will consider only the most elementary examples of half-BPS boundary conditions. The full classification and analysis of half-BPS boundary conditions with $\mathcal{N}=2$ supersymmetry is likely to be quite rich, just as in the case of $\mathcal{N}=4$ supersymmetry [48]. Here we will only scratch the surface.

In any local boundary conditions, half of the fermion fields will vanish at the boundary. The obvious condition that will accomplish this, while preserving all the symmetries of the matrix $M$, is to place on the fermions $\Psi$ in the vector multiplet a condition analogous to (3.68) but possibly with the opposite sign:

$$
M \Psi=z \Psi, \quad z= \pm 1 .
$$

For either choice of the sign $z$, we will describe a boundary condition on the bosons in the vector multiplet that preserves the supersymmetries with $M \eta=\eta$. 
The condition that a boundary condition preserves supersymmetry is that it ensures the vanishing of the normal component of the supercurrent at the boundary. The supercurrent is $J_{I}=\Gamma^{J K} \operatorname{Tr} F_{J K} \Gamma_{I} \Psi$, and so, bearing in mind that in our notation the normal coordinate is $x^{2}$, the condition that the normal part of $\bar{\eta} J_{I}$ vanishes at the boundary is that at the boundary we must have

$$
\bar{\eta} \operatorname{Tr} \Gamma^{J K} F_{J K} \Gamma_{2} \Psi=0 .
$$

To ensure this condition, we must pick a judicious boundary condition on the bosons $A_{I}$.

We find the condition we need by replacing $\bar{\eta}$ on the left hand side of (3.72) by $\bar{\eta} M$, moving $M$ to the right, and then using $M \Psi=z \Psi$. If $z=1$, then, since $M \Gamma_{2}=-\Gamma_{2} M$, the condition that we want is that the boundary values should obey

$$
\left[M, \Gamma^{J K} F_{J K}\right]=0
$$

If instead we take $z=-1$, then we need instead

$$
\left\{M, \Gamma^{J K} F_{J K}\right\}=0
$$

at the boundary.

To obey (3.73), we impose Dirichlet boundary conditions on $A_{4}$ and Neumann boundary conditions on the other components ${ }^{8}$ of $A$. (The former implies that $F_{I 4}=0, I \neq 2$, while the latter implies $F_{I 2}=0, I \neq 4$; together these imply (3.73).) And conversely, to obey (3.74), by an argument similar to the one just indicated, we place Neumann boundary conditions on $A_{4}$ and Dirichlet boundary conditions on the other components of $A$.

There is an important detail to mention here. There is never a problem with Dirichlet boundary conditions on gauge fields, but in four dimensions, Neumann boundary conditions on gauge fields are only possible if the gauge theory $\theta$-angles vanish. (Neumann boundary conditions in our notation would say that the boundary value of $F_{2 I}$ vanishes, $I=0,1,3$ while at non-zero $\theta$, one must add to this a multiple of $\theta \epsilon_{I J K} F_{J K}$. Here $I, J, K$ take values 013.) So at $\theta \neq 0$, we are limited to $z=-1$ and (3.74).

We can make these two boundary conditions more concrete as follows:

(I) In the first case (which as just explained requires the $\theta$-angles to vanish), $A_{4}$ vanishes on the boundary and $A_{1}, A_{3}$, and $A_{5}$ do not.

(II) In our second case, $A_{1}, A_{3}$, and $A_{5}$ vanish on the boundary and $A_{4}$ does not.

We have described these boundary conditions for vector multiplets, but they extend to hypermultiplets. In the case of a generalized quiver theory obtained by compactification from six dimensions on a Riemann surface $C$, the gauge group is semi-simple rather than simple. We pick the same type of boundary condition - type I or type II - for each factor.

Now let us describe what these boundary conditions look like after toroidal compactification to two dimensions. First we consider a free vector multiplet with gauge group

\footnotetext{
${ }^{8}$ From this and the other statements below, we omit $A_{2}$, the normal component of $A$, as it can be set to zero near the boundary by a gauge transformation.
} 
U(1). As usual, we $T$-dualize the holonomy of $A_{3}$ to a scalar $\varrho$. $\varrho$ obeys Dirichlet boundary conditions if $A_{3}$ obeys Neumann boundary conditions, and vice-versa.

So in case I, the fields that vanish at the boundary are $\varrho$ and $A_{4}$. We can think of $A_{4}$ as $\operatorname{Im} a_{D}$ where $a=A_{4}-i A_{5}$ and $a_{D}=\left(4 \pi i / e^{2}\right) a$. (We recall that for Type I, the gauge theory $\theta$-angle vanishes and so the gauge coupling parameter $\tau$ reduces to $4 \pi i / e^{2}$.) Thus, the Type I boundary condition leads to a brane supported on the Lagrangian submanifold $L_{1}$.

In case II, the fields that vanish on the boundary are $A_{1}$ and $A_{5}$. In the two-dimensional description, the fields that vanish on the boundary are $b$, which is the holonomy of $A_{1}$, and $\operatorname{Im} a$, which is a multiple of the holonomy of $A_{5}$. So the Type II boundary condition leads to a brane supported on the Lagrangian submanifold $L_{2}$.

What about the nonabelian case? We cannot so easily interpret the boundary conditions I and II in the low energy theory. But we can do so asymptotically on the Coulomb branch. Generically on the Coulomb branch, the gauge group is broken to an abelian subgroup; the only massless particles are $r$ vector multiplets, with $r$ the rank of the gauge group. Near infinity on the Coulomb branch (and far from the locus on which additional massless particles appear), an abelian treatment along the above lines is a good approximation.

So the brane $\mathcal{B}_{\mathrm{I}}$ coming from a Type I boundary condition can be described near infinity by the familiar conditions $\varrho^{i}=\operatorname{Im} a_{D}^{i}=0$. Similarly the brane $\mathcal{B}_{\text {II }}$ coming from a Type II boundary condition can be described near infinity by $b_{i}=\operatorname{Im} a_{i}=0$.

There are two reasons that we cannot simply imitate the derivation of eqs. (3.61) and (3.64) and determine the spectrum by setting $a_{i} / \varepsilon$ or $a_{D}^{i} / \varepsilon$ to integers. First, the branes $\mathcal{B}_{\text {I }}$ and $\mathcal{B}_{\text {II }}$ are described only near infinity by the conditions mentioned in the last paragraph. Second, in general the WKB approximation, which was used to arrive at (3.61) and (3.64), is not exact.

In section 2.1.1, we have described a situation in which the WKB approximation is exact. This involved a two-dimensional theory with $(2,2)$ supersymmetry, formulated on $\mathbb{R} \times S^{1}$. We have been studying here, after toroidal compactification from four dimensions, a theory with $(4,4)$ supersymmetry on $\mathbb{R} \times I$, with half-BPS boundary conditions at the ends. As far as we know, the WKB approximation is not exact in this situation.

\subsubsection{Alternative compactification to two dimensions}

However, we can look at the same problem in another way. Our starting point has been a four-dimensional gauge theory on $\mathbb{R} \times S^{1} \times D_{R, \varepsilon}$, understood in terms of $S^{1} \times \widetilde{S}^{1}$ compactification to two dimensions. This leads to a problem on $\mathbb{R} \times I$ that we have by now discussed at length.

However, a more obvious way to reduce the same problem to two dimensions is to simply view it as a compactification on $D_{R, \varepsilon}$ down to $\mathbb{R} \times S^{1}$. In this fashion, we arrive at a $(2,2)$ theory on $\mathbb{R} \times S^{1}$.

Let us consider the two cases of a Type I or Type II boundary condition. In the first case, all vector multiplets obey Neumann boundary condition. A four-dimensional vector multiplet with $\mathcal{N}=2$ supersymmetry reduces to a two-dimensional vector multiplet with $(2,2)$ supersymmetry. Four-dimensional hypermultiplets reduce to two-dimensional chiral 
multiplets. The net effect is that a four-dimensional generalized quiver theory reduces to a two-dimensional theory of $(2,2)$ supersymmetry based on the same generalized quiver.

This is precisely the setting for section 2.1.1, and the WKB approximation to the quantization will be exact when expressed in terms of the effective twisted chiral superpotential $\widetilde{W}$, which in general will depend on both the scalar fields in the vector multiplet and the deformation parameter $\varepsilon$. This twisted chiral superpotential has been analyzed in [7] and [6]. It receives contributions from perturbative effects and instanton effects near the tip of the cigar. General arguments concerning the $\Omega$-deformation show that all contributions come from the region near the tip. As far as we know, the analysis of $\widetilde{W}$ may as well be carried out in $\Omega$-deformed variables; we know of no advantage to the rotation to undeformed variables that has been exploited in the present paper.

Since the effective twisted chiral superpotential is the same as the one used in [6], the spectrum is also the same. All that we have gained by our approach here is the understanding of why this spectrum can be understood as coming from the quantization of a real slice of Hitchin's integrable system.

The case of a Type II boundary condition is a little different. All gauge fields obey Dirichlet boundary conditions. This completely breaks the gauge symmetry, so there are no gauge fields in the effective description on $\mathbb{R} \times S^{1}$. Both vector multiplets and hypermultiplets reduce to chiral multiplets in that effective description.

It seems that the clearest picture emerges if we rotate back from the ordinary variables that we have used in describing the boundary conditions to $\Omega$-deformed variables. This has the effect of exchanging $A_{4}$, which is a scalar field (the real part of $\phi$ ) with $A_{3}$, the component of the gauge field around $\widetilde{S}^{1}$, the boundary of $D_{R}$. So in this description, the boundary condition does not constrain the holonomy of $A_{3}$ around $\partial D_{R}$.

However, for the purposes of constructing a supersymmetric ground state, the holonomy around $\partial D_{R}$ must vanish, or supersymmetry will be violated by curvature in the interior of $D_{R}$. Treating $A_{3}$ as a constant in the low energy theory, the holonomy is $\exp \left(2 \pi \rho A_{3}\right)$, where $\rho$ is the radius of $\widetilde{S}^{1}$ in $\Omega$-deformed variables, so the condition is

$$
\exp \left(2 \pi \rho A_{3}\right)=1 \text {. }
$$

We want to rotate this condition back to undeformed variables. In doing so, we take $\rho \rightarrow \infty, \widehat{\rho} \rightarrow 1 / \varepsilon$, since this choice was built into our construction of boundary conditions in section 3.4.1. In the undeformed variables, $\rho A_{3}$ can be replaced by $A_{4} / \varepsilon$. So the condition becomes $\exp \left(2 \pi A_{4} / \varepsilon\right)=1$. The boundary condition also set $A_{5}=0$. We can combine the two statements to

$$
\exp (2 \pi \phi / \varepsilon)=1
$$

In the low energy description, we rotate $\phi$ to a maximal torus and denote it as $a$. Thus, (3.76) seems to show that the quantization of $a$ for a Type II boundary condition takes its most naive form, though this is not so for Type I.

\subsubsection{Eigenvalues and opers}

Finally, we will describe what may ultimately — after some future developments — be the most powerful way to get detailed results about quantization of these integrable systems. 
Something close to what we will describe momentarily has actually been carried out in the mathematical literature [19-22] in the context of an integrable system known as the Gaudin model. (This work has been done in a language much closer to geometric Langlands than to gauge theory, and at the moment we do not know precisely how to reformulate it in terms of gauge theory or even two-dimensional sigma-models.)

Our starting point in this section has been to choose a Lorentz-invariant boundary condition at the far end of $D_{R}$ that preserves supersymmetry of type $(A, B, A)$. In a description that arises by $T$-duality on scalars that represent holonomies around $\widetilde{S}^{1}$, this boundary condition determines a Lagrangian submanifold $L \subset \mathcal{M}_{H}$ and a corresponding Lagrangian brane $\mathcal{B}_{L}$. Quantization of $L$ is carried out by taking the space of $\left(\mathcal{B}_{\epsilon}, \mathcal{B}_{L}\right)$ strings.

However, at the far end of $D_{R}$, the two circles $S^{1}$ and $\widetilde{S}^{1}$ are on an equivalent footing. Hence, had we made the $T$-duality on scalars associated to $\widetilde{S}^{1}$ rather than $S^{1}$, the same boundary condition would have given a very similar Lagrangian submanifold $\widetilde{L}$ again of type $(A, B, A)$. Actually $\widetilde{L}$ is a Lagrangian submanifold not of $\mathcal{M}_{H}$ but of a dual moduli space $\widetilde{\mathcal{M}}_{H}$ obtained from $\mathcal{M}_{H}$ by $T$-duality on the fibers of the Hitchin fibration. ${ }^{9}$

What happens to the brane $\mathcal{B}_{\varepsilon}$ if we use a description obtained by $T$-duality on scalars coming from holonomy around $S^{1}$ rather than $\widetilde{S}^{1}$ ? In this case, $\mathcal{B}_{\varepsilon}$ is replaced by a new brane, also of type $(A, B, A)$, that arises from $\mathcal{B}_{\varepsilon}$ by $T$-duality on the fibers of the Hitchin fibration.

We will postpone a fuller explanation to section 4.7 , but in brief the dual of $\mathcal{B}_{\varepsilon}$ is a Lagrangian brane $\mathcal{B}_{N}$ supported on a Lagrangian submanifold $N$ that is known as the variety of opers. We can use this dual description to describe the space $\mathcal{H}$ of $\left(\mathcal{B}_{\varepsilon}, \mathcal{B}_{L}\right)$ strings; it is the same as the space of $\left(\mathcal{B}_{N}, \mathcal{B}_{\widetilde{L}}\right)$ strings. This space is easily described, assuming that the two Lagrangian submanifolds $N$ and $\widetilde{L}$ have generic (transverse) intersections. $\mathcal{H}$ has a basis ${ }^{10}$ with one basis vector for every intersection point of $N$ and $\widetilde{L}$.

The commuting Hamiltonians of the integrable system correspond to holomorphic functions on $\boldsymbol{N}$. The eigenvalues of the commuting Hamiltonians are simply the values of the corresponding functions at the points on $N$ at which $N$ intersects $\widetilde{L}$. Differently put, the joint eigenstates of the commuting Hamiltonians correspond to opers (points in $\boldsymbol{N}$ ) that obey a certain system of equations stating that they lie in $\widetilde{L}$. As remarked above, there is an example [19-22] of an integrable system whose spectrum has been described in just such a fashion.

\footnotetext{
${ }^{9}$ In the context of generalized quiver gauge theories, if $\mathcal{M}_{H}$ is a moduli space of Higgs bundles on a Riemann surface $C$ with gauge group $G$, then [45] $\widetilde{\mathcal{M}}_{H}$ is a corresponding moduli space of Higgs bundles on $C$ with gauge group $G^{\vee}$, the group dual to $G$. If $G$ is simply-laced, which is the case that arises most simply by reduction from the $(0,2)$ model in six dimensions, then $G$ and $G^{\vee}$ have the same universal cover, and so do $\mathcal{M}_{H}$ and $\widetilde{\mathcal{M}}_{H}$. In this situation, $L$ and $\widetilde{L}$ should be equivalent if lifted to the universal cover.

${ }^{10}$ In general, in the $A$-model of a symplectic manifold $X$, world-sheet instanton effects can remove from the cohomology the states corresponding to some intersections. In the present context, both branes $\mathcal{B}_{N}$ and $\mathcal{B}_{\widetilde{L}}$ are of type $(A, B, A)$, so they have extra supersymmetry in common beyond what is typical in an $A$-model. This extra supersymmetry generates an extra fermion zero mode in the field of an instanton, ensuring that instanton effects do not alter the cohomology that one reads off classically from the intersections of the two Lagrangian submanifolds.
} 


\section{Conformal blocks from four dimensions}

In this section, we will use similar methods to study a different problem - the relation [15] of four-dimensional gauge theory to Liouville theory and like theories in two dimensions.

\subsection{Gauge theory and Liouville theory}

We consider an $\mathcal{N}=2$ supersymmetric gauge theory in four dimensions on the fourmanifold $M^{\prime}=\mathbb{R} \times S^{3}$. Because there are now three curved dimensions in spacetime (as opposed to two in section 3 , where we worked on $M=\mathbb{R} \times S^{1} \times D_{R}$ ), topological twisting now leaves only two unbroken supercharges. This is so for any product metric on $\mathbb{R} \times S^{3}$, regardless of the choice of metric on $S^{3}$. One unbroken supercharge is the usual supercharge $Q$ that is related to Donaldson theory (in the case of $\mathrm{S} U(2)$ gauge theory without hypermultiplets). In Euclidean signature, the second supercharge $\bar{Q}$ can be obtained from the first by an orientation-reversing reflection of the first factor $\mathbb{R}$ of $M^{\prime}$. In Lorentz signature, $\bar{Q}$ is the hermitian adjoint of $Q$.

The twisting that preserves $Q$ and $\bar{Q}$ is defined in the usual way, by identifying the $\mathrm{S} U(2)_{R}$ global symmetry group with the structure group of the spin bundle of $S^{3}$. If one modifies the geometry of $M^{\prime}=\mathbb{R} \times S^{3}$ so as to be no longer a product, then the positive and negative chirality spin bundles of $M^{\prime}$ become distinct. For twisting, we must then decide whether we want to identify $\mathrm{SU}(2)_{R}$ with the structure group of the positive or negative chirality spin bundle. As a result, in the twisted theory with a non-product metric, we can conserve either $Q$ or $\bar{Q}$ but not both.

The most important special case of this arises if we "cap off" the metric on $M^{\prime}$. Topologically, we do this by viewing $S^{3}$ as the boundary of a ball $B^{4}$. Instead of a flat metric on $B^{4}$, we pick a metric that looks near the boundary like a product $\mathbb{R}^{-} \times S^{3}$, where $\mathbb{R}^{-}$is a half-line $t \leq 0$, but such that $S^{3}$ shrinks to a point at some $t=t_{0}<0$. In defining a topologically twisted theory on $B^{4}$ with such a metric, we can preserve $Q$ or $\bar{Q}$ but not both.

The basic property of $S^{3}$ that we will use is that it admits a $\mathrm{U}(1) \times \mathrm{U}(1)$ action. If $S^{3}$ is viewed as the locus $\sum_{i=1}^{4} y_{i}^{2}=1$ in $\mathbb{R}^{4}$, then we introduce polar coordinates $y_{1}+i y_{2}=u e^{i \alpha}$, $y_{3}+i y_{4}=v e^{i \beta}$ and finally $(u, v)=(\sin w, \cos w) . \mathrm{U}(1) \times \mathrm{U}(1)$ acts by shifts of $\alpha$ and $\beta$. The round metric on $S^{3}$ is $\mathrm{d} w^{2}+\sin ^{2} w \mathrm{~d} \alpha^{2}+\cos ^{2} w \mathrm{~d} \beta^{2}$. It will be convenient for us to use a more general $\mathrm{U}(1) \times \mathrm{U}(1)$-invariant metric on $S^{3}$. We let $w$ run over an interval $0 \leq w \leq \ell$, and we take

$$
\mathrm{d} s_{S^{3}}^{2}=\mathrm{d} w^{2}+f(w) \mathrm{d} \alpha^{2}+g(w) \mathrm{d} \beta^{2},
$$

where $f(w)=\rho_{1}^{2}$ except very near the left end-point $w=0$ where it vanishes quadratically,

and $g(w)=\rho_{2}^{2}$ except very near the right end-point $w=\ell$ where it vanishes quadratically. Here $\rho_{1}$ and $\rho_{2}$ are constants. In describing $S^{3}$ as in (4.1), we are in effect viewing it as a "warped" $T^{2}$ fibration over a one-dimensional base. On $M^{\prime}=\mathbb{R} \times S^{3}$, we take the product metric $\mathrm{d} s^{2}=\mathrm{d} t^{2}+\mathrm{d} s_{S^{3}}^{2}$. 


\section{2 $\Omega$-Deformation}

What we want to do with this theory is to $\Omega$-deform it, using the $\mathrm{U}(1) \times \mathrm{U}(1)$ symmetry. We follow the same basic procedure as in (3.20), but now we use the fact that four-dimensional gauge theories with $\mathcal{N}=2$ supersymmetry can arise by dimensional reduction from a sixdimensional theory with two more coordinates that we will here call $x^{4}$ and $x^{5}$. Apart from adding $\left(\mathrm{d} x^{4}\right)^{2}+\left(\mathrm{d} x^{5}\right)^{2}$ to the metric $\mathrm{d} s^{2}$, the only change that we make is to modify the terms that involve $\mathrm{d} \alpha$ and $\mathrm{d} \beta$. A simple case is a modification in which $\mathrm{d} \alpha$ "mixes" only with $\mathrm{d} x^{4}$ and $\mathrm{d} \beta$ "mixes" only with $\mathrm{d} x^{5}$, in the sense that the relevant part of the six-dimensional metric is

$$
f(w)\left(\mathrm{d} \alpha-\varepsilon_{1} \mathrm{~d} x^{4}\right)^{2}+g(w)\left(\mathrm{d} \beta-\varepsilon_{2} \mathrm{~d} x^{5}\right)^{2}+\left(\mathrm{d} x^{4}\right)^{2}+\left(\mathrm{d} x^{5}\right)^{2} .
$$

We have considered here a "diagonal" deformation in which $\alpha$ and $\beta$ mix only with $x^{4}$ and $x^{5}$, respectively. We take the deformation parameters $\varepsilon_{1}$ and $\varepsilon_{2}$ to be real and positive. In greater generality, as in [7], one can introduce complex deformation parameters $\epsilon_{1}, \epsilon_{2}$, set $x=x^{4}+i x^{5}$ and generalize (4.2) to

$$
f(w)\left(\mathrm{d} \alpha-\operatorname{Re}\left(\bar{\epsilon}_{1} \mathrm{~d} x\right)^{2}\right)+g(w)\left(\mathrm{d} \beta-\operatorname{Re}\left(\bar{\epsilon}_{2} \mathrm{~d} x\right)^{2}\right)+\left(\mathrm{d} x^{4}\right)^{2}+\left(\mathrm{d} x^{5}\right)^{2} .
$$

Evidently, the special case (4.2) corresponds to

$$
\epsilon_{1}=\varepsilon_{1}, \quad \epsilon_{2}=i \varepsilon_{2}
$$

and so

$$
\frac{\epsilon_{2}}{\epsilon_{1}}=i \frac{\varepsilon_{2}}{\varepsilon_{1}} .
$$

We will analyze here the special case of a diagonal deformation, and then in appendix A, we will analyze the general case using generalized complex geometry.

Let $S_{\varepsilon_{1}, \varepsilon_{2}}^{3}$ be $S^{3}$ with the $\Omega$-deformation with the indicated parameters. We let $\mathcal{H}_{\varepsilon_{1}, \varepsilon_{2}}$ be the space of supersymmetric ground states of the $\Omega$-deformed theory on $\mathbb{R} \times S_{\varepsilon_{1}, \varepsilon_{2}}^{3}$. We interpret the results of [15] to mean that $\mathcal{H}_{\varepsilon_{1}, \varepsilon_{2}}$ can be identified, for $G=\mathrm{S} U(2)$, with the space of Virasoro conformal blocks on the Riemann surface $C$. (Virasoro conformal blocks are the conformal blocks of Liouville theory. If $\mathrm{S} U(2)$ is replaced by another simply-laced group $G$, then $[43,44]$ the relevant objects are the conformal blocks of the corresponding Toda field theory.) Understanding this claim and certain related facts will be our goal in the rest of this paper.

Our first step will be to follow the logic of section 3.2.2 and compare the $\Omega$-deformed description to a standard one. In the region in which $f$ and $g$ are constants, the $\Omega$ deformation can be removed by an ordinary change of variables. The combined operation of $\Omega$-deformation plus ordinary change of variables amounts to a rotation of the $\alpha 4$ plane times a rotation of the $\beta 5$ plane, each of them precisely analogous to (3.34). (The more general deformation (4.3) can also be removed by a change of variables in the region in which $f$ and $g$ are constants, though the necessary formulas are more complicated.)

Just as in section 3.2.2, the simplest situation is that in which the radii $\rho_{1}, \rho_{2}$ in the $\Omega$-deformed description are taken to infinity, keeping the deformation parameters $\varepsilon_{i}$ 
fixed. Then the rotation that goes from $\Omega$-deformed description to the one by conventional variables is simply the product of $\pi / 2$ rotations in the $\alpha 4$ and $\beta 5$ planes. In the undeformed language, the radii of the circles parametrized by $\alpha$ and $\beta$ are

$$
\widehat{\rho}_{1}=\frac{1}{\varepsilon_{1}}, \quad \widehat{\rho}_{2}=\frac{1}{\varepsilon_{2}} .
$$

This means that the $\tau$ parameter of the torus parametrized by $\alpha, \beta$ is in the undeformed description

$$
\widehat{\tau}=i \frac{\varepsilon_{2}}{\varepsilon_{1}}=\frac{\epsilon_{2}}{\epsilon_{1}} .
$$

(We denote this $\tau$ parameter as $\widehat{\tau}$ to avoid confusion with generic coupling parameters of an $\mathcal{N}=2$ supersymmetric gauge theory, or complex structure parameters of a Riemann surface $C$, which we have called $\tau$.)

Extending (3.37), the gauge coupling in the ordinary description is

$$
\widehat{e}^{2}=\frac{e^{2}}{\sqrt{\left(1+\varepsilon_{1}^{2} \rho_{1}^{2}\right)\left(1+\varepsilon_{2}^{2} \rho_{2}^{2}\right)}} .
$$

In particular, $\widehat{e}^{2} \rightarrow 0$ as $\rho_{1}, \rho_{2} \rightarrow \infty$.

The $\pi / 2$ rotations means that in transforming from the $\Omega$-deformed description to the ordinary description, the supersymmetry generator $\eta$ is transformed by

$$
\eta \rightarrow \frac{1+\Gamma_{\alpha 4}}{\sqrt{2}} \frac{1+\Gamma_{\beta 5}}{\sqrt{2} \eta}
$$

\subsection{Two-dimensional description}

Just as in section 3 , we want to think of $M^{\prime}=\mathbb{R} \times S^{3}$ as an $S^{1} \times \widetilde{S}^{1}$ fibration, with fiber parametrized by $\alpha, \beta$, over $\mathbb{R} \times I$, parametrized by $t, w$. As in section 3 , there are branes at both ends of $I$. In section 3 , just one of these branes originated from the geometry at the tip of a cigar. But in our present problem, both branes have such an origin. In fact, near either end of $I, M^{\prime}$ resembles, up to a fairly obvious change of variables, the cigar geometry that led to our friend $\mathcal{B}_{\varepsilon}$.

This will enable us to borrow the analysis of section 3 , but we do have to keep in mind that the isomorphism with section 3 is different at the two ends of $I$. Near $w=0$, the $\alpha$ circle is shrinking and thus corresponds to $\widetilde{S}^{1}$ in section 3 . The coordinates $(t, w, \alpha, \beta)$ should be matched near $w=0$ with $\left(x^{0}, x^{2}, x^{3}, x^{1}\right)$ in section 3 . Near $w=\ell$, the $\beta$ circle shrinks and corresponds to $\widetilde{S}^{1}$; the roles of $\alpha$ and $\beta$ are exchanged.

We will denote as $\mathcal{B}_{\alpha}$ the brane at $w=0$ where the $\alpha$ circle shrinks, and as $\mathcal{B}_{\beta}$ the brane at $w=\ell$ where the $\beta$ circle shrinks. Let us first understand the brane $\mathcal{B}_{\alpha}$ in terms of ordinary variables.

Since the geometry near $w=0$ is the same as the geometry near $x^{2}=0$ in section 3 , the only reason that the brane $\mathcal{B}_{\alpha}$ is not trivially equivalent to the brane $\mathcal{B}_{\varepsilon}$ studied in section 3 is that a rotation of observables must be made to compare the two. For $\mathcal{B}_{\alpha}$, the rotation that we want to make from $\Omega$-deformed to ordinary variables is by the product

$$
\frac{1+\Gamma_{\alpha 4}}{\sqrt{2}} \frac{1+\Gamma_{\beta 5}}{\sqrt{2}}
$$


which in the notation of section 3 corresponds to

$$
\frac{1+\Gamma_{34}}{\sqrt{2}} \frac{1+\Gamma_{15}}{\sqrt{2}}
$$

By contrast, in section 3 , we made the rotation by $\left(1+\Gamma_{34}\right) / \sqrt{2}$. So in comparing $\mathcal{B}_{\varepsilon}$ to $\mathcal{B}_{\alpha}$, we need to make an additional rotation of the supersymmetry parameter $\eta$ (and all other operators and observables) by $\left(1+\Gamma_{15}\right) / \sqrt{2}$, whose effect is to exchange $\Gamma_{1}$ and $\Gamma_{5}$.

With this in mind, we re-examine some of the key equations of section 3.3. The supersymmetries preserved by $\mathcal{B}_{\varepsilon}$ were characterized by eq. (3.43), and as the matrix on the left hand side commutes with $\Gamma_{15}$, the brane $\mathcal{B}_{\alpha}$ preserves the same supersymmetries as $\mathcal{B}_{\varepsilon}$. Likewise, the condition (3.44) that characterizes which supersymmetries of a given brane can be interpreted in two-dimensional topological field theory is again defined with a matrix that commutes with $\Gamma_{15}$. So $\mathcal{B}_{\alpha}$ is a brane of type $(A, B, A)$, just like $\mathcal{B}_{\varepsilon}$.

The only difference between the two branes is that the 15 rotation acts non-trivially on the $\mathbb{C P}^{1}$ that parametrizes the possible topological field theory structure. Eq. (3.45), which singles out the supersymmetry generator of the $B$-model in complex structure $J$, is again invariant under the 15 rotation. But the other two conditions (3.46) and (3.47), which characterize the $A$-models of types $\omega_{K}$ and $\omega_{I}$, are exchanged under this rotation. ${ }^{11}$

The important consequence of this is that the supersymmetry charge $Q$ that is related to instanton counting and Donaldson theory, and which in section 3 was interpreted in undeformed variables as the generator of the $A$-model supersymmetry of type $\omega_{K}$, will now have to be interpreted as the generator of the $A$-model supersymmetry of type $\omega_{I}$. This is the supercharge we care about for our present purposes, since it is the only one (apart from its adjoint) that is conserved in the twisted theory on the full $\mathbb{R} \times S^{3}$ geometry.

Now let us consider our other brane $\mathcal{B}_{\beta}$, the one at $w=\ell$. All the same reasoning applies except that $x^{4}$ is exchanged with $x^{5}$. This exchanges the scalar field $A_{4}$ with $A_{5}$. That exchange can be carried out by a $\mathrm{U}(1)_{R}$ transformation which also rotates complex structure $J$ into $K$ (with a sign mentioned in footnote 11) while fixing $I$. So $\mathcal{B}_{\beta}$ is a brane of type $(A, A, B)$. (The $\mathrm{U}(1)_{R}$ transformation that maps $A_{4}$ to $A_{5}$ may not be a symmetry, as it also acts on mass parameters in an underlying four-dimensional Lagrangian. But still it can be used to determine the type of supersymmetry preserved by $\mathcal{B}_{\beta}$, given that we know this for $\mathcal{B}_{\alpha}$.) The same reasoning as before shows that the usual supercharge $Q$ of instanton counting and Donaldson theory is associated to the $A$-model of type $\omega_{I}$.

Obviously, the only structure that a brane $\mathcal{B}_{\alpha}$ of type $(A, B, A)$ and a brane $\mathcal{B}_{\beta}$ of type ( $A, A, B)$ have in common is the $A$-model of type $\omega_{I}$. Everything hangs together, since the supersymmetry generator of this $A$-model is indeed the supercharge $Q$ that is conserved in the $\mathbb{R} \times S^{3}$ geometry.

\subsection{The Kahler parameter of the sigma model}

Of the three Kahler classes on $\mathcal{M}_{H}, \omega_{I}$ is topologically non-trivial, but $\omega_{J}$ and $\omega_{K}$ are topologically trivial. This has been explained in section 2.3.2.

\footnotetext{
${ }^{11}$ To be more precise, there is a minus sign here, and the mapping takes $\left(\omega_{I}, \omega_{K}\right) \rightarrow\left(\omega_{K},-\omega_{I}\right)$. Similarly, two paragraphs below, the exchange of $J$ and $K$ is really $(J, K) \rightarrow(K,-J)$.
} 
Accordingly, the $A$-models of type $\omega_{J}$ or $\omega_{K}$ have no coupling parameter. But the $A$-model of type $\omega_{I}$ does have such a parameter, which we can think of as the Kahler parameter in complex structure $I$. According to a standard result $[36,37]$ that was explained in section 3.1.4, this parameter, assuming its imaginary part is positive, is the modular parameter $\widehat{\tau}$ of the two-torus $T^{2}$ that is parametrized by the angles $\alpha, \beta$, measured in the metric that is appropriate for the undeformed description. ${ }^{12}$ That modular parameter was computed in eq. (4.7):

$$
\widehat{\tau}=i \frac{\varepsilon_{2}}{\varepsilon_{1}}=\frac{\epsilon_{2}}{\epsilon_{1}} .
$$

So far we have only deduced that the model is the $A$-model of type $I$ with this $\widehat{\tau}$ parameter for real $\varepsilon_{1}, \varepsilon_{2}$. However, this must be true in general by holomorphy. This will be shown more explicitly in appendix A using generalized complex geometry.

In particular, the exchange of the $\alpha$ and $\beta$ circles corresponds to $\widehat{\tau} \rightarrow 1 / \widehat{\tau}$. One might expect that there would be a minus sign in this formula, so let us examine this point closely. A simple exchange $\alpha \leftrightarrow \beta$ (with no minus signs) gives an orientation-preserving automorphism of the $S^{3}$ metric (4.1) if accompanied by $w \rightarrow \ell-w$. A look at (4.3) shows that to extend this operation to a symmetry of the $\Omega$-deformed theory, we must take $\epsilon_{1} \leftrightarrow \epsilon_{2}$, again with no minus signs. To get a symmetry that preserves the overall orientation of $\mathbb{R} \times S_{\alpha, \beta}^{3}$, we take it to act trivially on $\mathbb{R}$. We call the combined operation $\boldsymbol{W}$. It acts on $\widehat{\tau}$ by $\widehat{\tau} \rightarrow+1 / \widehat{\tau}$.

$W$ has an interesting interpretation in the context of generalized quiver gauge theories. Consider a four-dimensional $\mathcal{N}=2$ theory obtained as in [16] by compactifying the six-dimensional $(0,2)$ theory on a Riemann surface $C$. Then compactify further to two dimensions on the $\alpha \beta$ torus $T^{2}$.

Altogether, we are compactifying from six to two dimensions on $T^{2} \times C$. Introducing an interval $I$ parametrized by $w$, the full spacetime, away from the boundaries of $I$, is $\mathbb{R} \times I \times C \times T^{2}$. If we carry out first the compactification on $T^{2}$, we get $\mathcal{N}=4$ super Yang-Mills theory on $\mathbb{R} \times I \times C$. The orientation-preserving mapping class group of $T^{2}$ is $\mathrm{S} L(2, \mathbb{Z})$, and this is usually called the electric-magnetic duality group in four dimensions. However, if we allow diffeomorphisms of $T^{2}$ that reverse its orientation, we get an extended mapping class group $G L(2, \mathbb{Z})$. Orientation-reversing symmetries of $T^{2}$ lead to symmetries of $\mathcal{N}=4$ super Yang-Mills theory if accompanied by orientation-reversing symmetries of spacetime. In the present case, $\boldsymbol{W}$ acts by $\alpha \leftrightarrow \beta$, which reverses the orientation of $T^{2}$, and is accompanied by a transformation $w \rightarrow \ell-w$ which reverses the orientation of $\mathbb{R} \times I \times C$.

We can relate what we have found to a more familiar symmetry $\widehat{\tau} \rightarrow-1 / \widehat{\tau}$. Let $\boldsymbol{T}$ be the combination $(\alpha, \beta) \rightarrow(-\alpha, \beta)$ together with time-reversal acting on $\mathbb{R}$ and the identity on $w$. Then $\boldsymbol{T}$ preserves the orientation of $\mathbb{R} \times S_{\alpha, \beta}^{3}$. It acts on the deformation parameters by $\left(\epsilon_{1}, \epsilon_{2}\right) \rightarrow\left(-\epsilon_{1}, \epsilon_{2}\right)$. So the combination $\boldsymbol{T} \boldsymbol{W}$ acts on the deformation parameters by $\left(\epsilon_{1}, \epsilon_{2}\right) \rightarrow\left(\epsilon_{2},-\epsilon_{1}\right)$, or $\widehat{\tau} \rightarrow-1 / \widehat{\tau}$.

\footnotetext{
${ }^{12}$ As was explained in section 2.3.2 and as we further explain at the end of the present subsection, this Kahler parameter is complex-valued, with no condition of positivity, but we have to use a different description when its imaginary part is not positive.
} 
In the description by $\mathcal{N}=4$ super Yang-Mills theory on $\mathbb{R} \times I \times C, \boldsymbol{T}$ is time-reversal, acting as -1 on $\mathbb{R}$ and trivially on $I \times C$. The composition $\boldsymbol{T} \boldsymbol{W}$ preserves the orientation of $\mathbb{R} \times I \times C$, and acts on $\widehat{\tau}$ as a standard electric-magnetic duality transformation $\widehat{\tau} \rightarrow-1 / \widehat{\tau}$.

According to [13], in the context of compactification from four to two dimensions on a Riemann surface $C$, the symmetry $\widehat{\tau} \rightarrow-1 / \widehat{\tau}$ is the basis for geometric Langlands duality. The most basic application of this duality to the geometric Langlands program involves the duality between the $B$-model of $\mathcal{M}_{H}$ in complex structure $J$ and the $A$-model of type $\omega_{K}$. Section 3 of the present paper was based on the $A$-model of $\mathcal{M}_{H}$ of type $\omega_{K}$, but its duality with the $B$-model of type $J$ did not play an important role.

However, geometric Langlands duality has an extension ${ }^{13}$ involving a complex parameter that was called $\Psi$ in [13]. As explained in section 5.2 of [13], extended geometric Langlands can be naturally described by the $A$-model of $\mathcal{M}_{H}$ in symplectic structure $\omega_{I}$, with the modulus $\widehat{\tau}$ of that $A$-model equal to $\Psi$. The operation $\boldsymbol{T W}$ amounts to the extended geometric Langlands duality $\Psi \rightarrow-1 / \Psi$. Thus, the exchange of the two circles, which certainly will be important in our analysis in the rest of this paper, is the basis for extended geometric Langlands duality.

In the $A$-model of type $\omega_{I}$ as usually defined, $\widehat{\tau}=\Psi$ is a Kahler parameter that takes values in the upper half of the complex plane. However, according to [13], and as we explained in relation to eq. (2.19), in a more complete description, $\Psi$ actually parametrizes $\mathbb{C P}^{1}$. Values of $\Psi$ in the lower half plane can be reached by an $A$-model of symplectic structure $-\omega_{I}$. The cases that $\Psi$ is real or $\infty$ are more subtle. The case of real $\Psi$ can be studied as an $A$-model with symplectic form $\omega_{K}$ and a $B$-field proportional to $\omega_{I}$, while $\Psi=\infty$ is the $B$-model of type $J$.

\subsection{More about the branes}

Now let us discuss in more detail the branes that appear in our $T^{2}$ compactification to $\mathbb{R} \times I$.

To arrive at a conventional sigma-model on $\mathbb{R} \times I$, we must make a $T$-duality on the scalars that arise from gauge field holonomies on either the $\alpha$ circle or the $\beta$ circle. Suppose that we make the $T$-duality associated to the $\alpha$ circle. Then as explained in section 4.3, the brane $\mathcal{B}_{\alpha}$ is a rotated version of the brane of type $(A, B, A)$ studied in section 3.

If we make the opposite $T$-duality on the $\beta$ circle, the other brane $\mathcal{B}_{\beta}$ can be described similarly; it is the analogous space-filling rank 1 brane of type $(A, A, B)$. By a $\mathrm{U}(1)_{R}$ chiral rotation (which in general will transform the mass and scale parameters of the theory), we can rotate $K$ back to $J$ and arrive at a description just like the one in the last paragraph. Thus, $\mathcal{B}_{\beta}$ in one description is equivalent to $\mathcal{B}_{\alpha}$ in the other description, up to the chiral rotation.

However, our goal is to understand the physical Hilbert space $\mathcal{H}_{\varepsilon_{1}, \varepsilon_{2}}$ of an underlying four-dimensional $\mathcal{N}=2$ theory compactified on $S^{3}$ with an $\Omega$-deformation. From a twodimensional viewpoint, $\mathcal{H}$ is the space of $\left(\mathcal{B}_{\alpha}, \mathcal{B}_{\beta}\right)$ strings. To describe this space, we need to describe both branes in the same language.

\footnotetext{
${ }^{13}$ In the mathematical literature, this extension is often called quantum geometric Langlands, but we will avoid this terminology because we interpret also "classical" geometric Langlands via quantum field theory.
} 


\begin{tabular}{|c|c|}
\hline Model & Dual \\
\hline$I_{B}$ & $I_{B}$ \\
$I_{A}$ & $I_{A}$ \\
$J_{B}$ & $K_{A}$ \\
$J_{A}$ & $K_{B}$ \\
$K_{B}$ & $J_{A}$ \\
$K_{A}$ & $J_{B}$ \\
\hline
\end{tabular}

Table 1. Listed here are the $A$ - and $B$-models to which a given model transforms under $T$-duality on the fibers of the Hitchin fibration. For example, the last row asserts that $K_{A}$, the $A$-model of type $\omega_{K}$, is mapped to $J_{B}$, the $B$-model of type $J$.

We have two options. We can describe $\mathcal{B}_{\alpha}$ via a $T$-duality associated to the $\beta$ circle rather than the $\alpha$ circle. This will turn $\mathcal{B}_{\alpha}$ into a Lagrangian brane $\mathcal{B}_{N}$ of type $(A, B, A)$ that we will describe.

Or conversely we can describe $\mathcal{B}_{\beta}$ via a $T$-duality on the $\alpha$ circle. In this description, $\mathcal{B}_{\beta}$ turns into a Lagrangian brane $\mathcal{B}_{N^{\prime}}$ of type $(A, A, B)$ that can be reached from $\mathcal{B}_{N}$ by a $\mathrm{U}(1)_{R}$ rotation.

The space $\mathcal{H}$ has two dual descriptions. It is the space of $\left(\mathcal{B}_{N}, \mathcal{B}_{\beta}\right)$ strings in the $A$ model of type $\omega_{I}$ with coupling parameter $\widehat{\tau}=i \varepsilon_{1} / \varepsilon_{2}$, or the space of $\left(\mathcal{B}_{\alpha}, \mathcal{B}_{N^{\prime}}\right)$ strings in the "same" model but with $\widehat{\tau}=i \varepsilon_{2} / \varepsilon_{1}$.

We have put quotes around the word "same" because there is actually a duality involved. The two descriptions differ by the combined $T$-duality associated to the $\alpha$ and $\beta$ circles (which we recall is the basic geometric Langlands duality); this is the same as a $T$-duality on the fibers of the Seiberg-Witten fibration. In the simplest generalized quiver theories that originate in six dimensions, the Seiberg-Witten fibration is the Hitchin fibration for Higgs bundles with a simply-laced gauge group $G$. Under duality on the fibers of the Hitchin fibration, the structure group $G$ of the Hitchin fibration is mapped to $G^{\vee}$. (This was first shown in [45].) Since $G$ is simply-laced, $G$ and $G^{\vee}$ have the same universal cover; if one is $\mathrm{S} U(2)$, the other is $\mathrm{SO}(3)$. Still, the distinction between $G$ and $G^{\vee}$ is significant in a very precise description.

The combined $T$-duality on the $\alpha$ and $\beta$ circles maps $\mathcal{B}_{\alpha}$ to $\mathcal{B}_{N}$ and $\mathcal{B}_{\beta}$ to $\mathcal{B}_{N^{\prime}}$. How it acts on the supersymmetries is summarized in the table. For example, the first row of the table asserts that the $B$-model of type $I$ - denoted in the table as $I_{B}$ - is mapped to itself by the $T$-duality on the fibers of the Hitchin fibration. This reflects the fact that the Hitchin fibration is holomorphic in complex structure $I$. Similarly, the $B$-model of type $J$ - denoted $J_{B}$ - is exchanged with the $A$-model of type $\omega_{K}$ - denoted $K_{A}$. The table is explained in section 5 of [13].

\subsection{A practice case}

Before discussing the duals of the branes $\mathcal{B}_{\alpha}$ and $\mathcal{B}_{\beta}$, we will first practice with a simpler but still subtle case. This simpler case is the brane $\mathcal{B}_{*}$ of type $(B, B, B)$ whose support is 
all of $\mathcal{M}_{H}$ and whose Chan-Paton bundle is trivial, by which we mean in particular that it is flat.

Since the Chan-Paton bundle of $\mathcal{B}_{*}$ is flat, it is certainly flat when restricted to each fiber of the Hitchin fibration. So a fiber of the Hitchin fibration is mapped by the duality to a point, and hence the $T$-dual $\mathcal{B}_{N^{*}}$ of $\mathcal{B}_{*}$ is supported on a section $\boldsymbol{N}^{*}$ of the Hitchin fibration (of the dual group $G^{\vee}$ ). $\boldsymbol{N}^{*}$ is automatically middle-dimensional, and as $\mathcal{B}_{N^{*}}$ is a brane of type $(B, A, A)$, it is holomorphic in complex structure $I$ and Lagrangian with respect to $\Omega_{I}=\omega_{J}+i \omega_{K}$.

The section $\boldsymbol{N}^{*}$ of the Hitchin fibration is actually almost uniquely determined (up to a choice in what follows of a spin structure on $C$ ) by the fact that it is holomorphic in complex structure $I$. How to construct such a holomorphic section was shown in [17]. We will describe the construction only for $G=\mathrm{S} U(2)$. We think of $\mathcal{M}_{H}$ as a moduli space of stable Higgs bundles, that is pairs $(E, \varphi)$ where $E$ is a rank two holomorphic vector bundle over $C$ of trivial determinant, and $\varphi$ is a holomorphic section of $K_{C} \otimes \operatorname{ad}(E)$. To describe a holomorphic section of the Hitchin fibration, we pick a square root $K_{C}^{1 / 2}$, and take $E=K_{C}^{-1 / 2} \oplus K_{C}^{1 / 2}$. This is the most unstable bundle $E$ for which there exists a stable Higgs bundle $(E, \varphi)$. Requiring $(E, \varphi)$ to be stable, the most general choice of $\varphi$ up to an automorphism of $E$ is

$$
\varphi=\left(\begin{array}{ll}
0 & 1 \\
v & 0
\end{array}\right)
$$

where $v$ is a quadratic differential on $C$ and we regard $\varphi$ as a matrix acting on $\left(\begin{array}{c}K_{C}^{-1 / 2} \\ K_{C}^{1 / 2}\end{array}\right)$. For $G=\mathrm{S} U(2)$, the fibers of the Hitchin fibration are parametrized by the value of $\operatorname{Tr} \varphi^{2}$. Since $\operatorname{Tr} \varphi^{2}=2 v$, there is one choice of $v$ for any desired value of $\operatorname{Tr} \varphi^{2}$. Hence this family of Higgs bundles $(E, \varphi)$, which we will call $\boldsymbol{N}^{*}$, gives a section of the Hitchin fibration. This section is manifestly holomorphic in complex structure $I$. To show that $N^{*}$ is Lagrangian for the holomorphic two-form $\Omega_{I}$, we use the explicit formula

$$
\Omega_{I}=\delta\left(\frac{1}{\pi} \int_{C} \operatorname{Tr} \phi_{z} \delta A_{\bar{z}}\right) .
$$

Since $E$, which is characterized by $A_{\bar{z}}$, is fixed in the family $\boldsymbol{N}^{*}$, we can take $\delta A_{\bar{z}}=0$ when restricted to $N^{*}$. So a brane $\mathcal{B}_{N^{*}}$ supported on $N^{*}$ with trivial Chan-Paton bundle is a brane of type $(B, A, A)$, as expected.

Continuing with the case $G=\mathrm{S} U(2), \boldsymbol{N}^{*}$ can be naturally identified with the Teichmuller space $C$ of the Riemann surface $C$. (This is proved [17] by showing that $N^{*}$ can be interpreted as a component of the moduli space of flat $\mathrm{S} L(2, \mathbb{R})$ bundles on $C$.) In fact, the symplectic form $\omega_{I}$ of $\mathcal{M}_{H}$, restricted to $N^{*}$, and with the standard normalization eq. (2.18), is the natural Weil-Petersson symplectic form of Teichmuller space. Since the coupling parameter of our $A$-model is $\widehat{\tau}=i \varepsilon_{1} / \varepsilon_{2}$, the symplectic form of the $A$-model is $\varepsilon_{1} / \varepsilon_{2}$ times the Weil-Petersson form.

The relation of $\omega_{I}$ to the Weil-Petersson form makes possible the following construction, described in section 4 of [14]. Let $\mathcal{B}^{\prime}$ be the brane with support all of $\mathcal{M}_{H}$ and Chan-Paton curvature $\omega_{I}^{*}=\left(\varepsilon_{1} / \varepsilon_{2}\right) \omega_{I}$. So $\mathcal{B}^{\prime}$ is a coisotropic brane of type $(B, A, A)$. 
Viewing $\mathcal{B}^{\prime}$ and $\mathcal{B}_{N^{*}}$ as $A$-branes of type $\omega_{K}$, let $\mathcal{H}^{\prime}$ be the space of $\left(\mathcal{B}^{\prime}, \mathcal{B}_{N^{*}}\right)$ strings. As reviewed in section 2.3, $\mathcal{H}^{\prime}$ can be interpreted as quantization of $\boldsymbol{N}^{*}$ with symplectic structure $\omega_{I}^{*}$ - in other words, quantization of Teichmuller space with symplectic form $\varepsilon_{1} / \varepsilon_{2}$ times the Weil-Petersson form.

Now let us think back to the problem of four-dimensional gauge theory that has motivated our analysis in this section: how to relate the space $\mathcal{H}_{\varepsilon_{1}, \varepsilon_{2}}$ of supersymmetric ground states of a rank 1 generalized quiver theory on $\mathbb{R} \times S_{\varepsilon_{1}, \varepsilon_{2}}^{3}$ to the space of Virasoro (or Liouville) conformal blocks on $C$. Teichmuller space has been quantized [46] using a real polarization (which depends on the choice of a set of $A$-cycles on $C$ ) in a way that certainly appears to give a good candidate for the space of Virasoro conformal blocks. (There is also an analog for groups of higher rank [47].) If therefore we are aiming to get the space of Virasoro conformal blocks as the space of ground states of open strings stretched between two branes, then $\mathcal{B}^{\prime}$ and $\mathcal{B}_{N^{*}}$ would appear to be good candidates for those branes.

These are, however, not the candidates that have emerged from our analysis. We have found instead that $\mathcal{H}_{\varepsilon_{1}, \varepsilon_{2}}$ is the space of $\left(\mathcal{B}_{\alpha}, \mathcal{B}_{N^{\prime}}\right)$ strings (or the space of $\left(\mathcal{B}_{N}, \mathcal{B}_{\beta}\right)$ strings). Here $\mathcal{B}_{\alpha}$ is related to the more naive candidate $\mathcal{B}^{\prime}$ by a sort of hyper-Kahler rotation; one is a rank one coisotropic brane of type $(A, B, A)$, and the other is a similar object of type $(B, A, A)$. Similarly, $\mathcal{B}_{\boldsymbol{N}^{\prime}}$ is related to $\mathcal{B}_{N^{*}}$ by a sort of dual hyper-Kahler rotation. We do not have an intuitive understanding of why our construction has led to the space of $\left(\mathcal{B}_{\alpha}, \mathcal{B}_{N^{\prime}}\right)$ strings rather than the more obvious space of $\left(\mathcal{B}^{\prime}, \mathcal{B}_{N^{*}}\right)$ strings. Perhaps these spaces are actually naturally isomorphic.

The space of $\left(\mathcal{B}^{\prime}, \mathcal{B}_{N^{*}}\right)$ strings, since it describes quantization of a component of the moduli space of flat $\mathrm{S} L(2, \mathbb{R})$ connections on the Riemann surface $C$, certainly appears to be related to $\mathrm{S} L(2, \mathbb{R})$ Chern-Simons gauge theory in three dimensions. The construction also has an analog [14] that is similarly related to $\mathrm{S} U(2)$ Chern-Simons gauge theory in $2+1$ dimensions. In this analog, $\boldsymbol{N}^{*}$ is replaced by the locus of flat $\mathrm{SU}(2)$ bundles. This locus is characterized by the condition $\varphi=0$ and is, like $N^{*}$, the support of a Lagrangian brane of type $(B, A, A)$.

\subsection{The brane of opers}

Now we want to discuss the dual of $\mathcal{B}_{\alpha}$ (or equivalently, modulo a $\mathrm{U}(1)_{R}$ rotation, the dual of $\left.\mathcal{B}_{\beta}\right)$. The Chan-Paton curvature of $\mathcal{B}_{\alpha}$ is not zero; rather it equals $\omega_{J}^{*}=\left(\varepsilon_{1} / \varepsilon_{2}\right) \operatorname{Re} \Omega_{I}$. However, as the fibers of the Hitchin fibration are Lagrangian for $\Omega_{I}$, the Chan-Paton bundle of $\mathcal{B}_{\alpha}$ is flat when restricted to a fiber of that fibration.

This means that when restricted to a fiber, the $T$-dual of $\mathcal{B}_{\alpha}$ is a point. Therefore, the $T$-dual of $\mathcal{B}_{\alpha}$ is supported on a section of the Hitchin fibration. We call this section $\boldsymbol{N}$ and we write $\mathcal{B}_{N}$ for the $T$-dual of $\mathcal{B}_{\alpha}$.

$\mathcal{B}_{N}$ is a brane of type $(A, B, A)$, and as its support is middle-dimensional, it is a Lagrangian brane. Hence the Chan-Paton bundle of $\mathcal{B}_{N}$ is flat. It therefore is actually trivial, since $N$, being a section of the Hitchin fibration, is equivalent topologically to the base $\boldsymbol{B}$ and so is contractible.

$N$ is different from the section $N^{*}$ of the Hitchin fibration that was described in eq. (4.13), since they are holomorphic in different complex structures. $\boldsymbol{N}^{*}$ is holomorphic 
in complex structure $I$ but $N$ is holomorphic in complex structure $J$. The Hitchin fibration is holomorphic only in complex structure $I$, and the fact that this is the complex structure in which $N^{*}$ is holomorphic makes $N^{*}$ much simpler to study than $N$.

Nevertheless, an explicit description of $\boldsymbol{N}$ is known, in essence, from work on geometric Langlands [33]. (In addition, the dual of $\mathcal{B}_{\alpha}$ has been described in four-dimensional gauge theory language in section 4 of [48]. It should be possible to reduce this description to two dimensions and recover the result of [33], though this has not yet been done.) Since we do not know a quick path to this description and will not use the details in the rest of this paper, we will here simply state the result.

We describe the result only for gauge group $G=\mathrm{S} U(2)$. Also we assume that $C$ has a negative Euler class (if its genus is 0 or 1 , we assume it has at least 3 or 1 marked points, respectively). If $E \rightarrow C$ is a flat bundle of rank 2, it can in particular be regarded as a holomorphic bundle. A flat bundle is called an "oper" if, viewed as a holomorphic bundle, it is a non-trivial extension

$$
0 \rightarrow K_{C}^{1 / 2} \rightarrow E \rightarrow K_{C}^{-1 / 2} \rightarrow 0
$$

where $K_{C}^{1 / 2}$ is a square root of the canonical bundle of $C$. For a given choice of $K_{C}^{1 / 2}$, a non-trivial extension of this kind is unique up to isomorphism. If $E$ is a rank 2 bundle with flat connection $\mathcal{A}$ that is an oper, then the $(0,1)$ part of $\mathcal{A}$ has the form

$$
\mathcal{A}_{\bar{z}}=\left(\begin{array}{cc}
-a_{\bar{z}} & 0 \\
u & a_{\bar{z}}
\end{array}\right),
$$

where $a_{\bar{z}}$ defines the complex structure of the line bundle $K_{C}^{1 / 2}$, and $u$ is a $K_{C}$-valued $(0,1)$-form; the choice of $u$ does not matter, up to gauge transformation, as long as its cohomology class in $\left.H^{1}\left(C, K_{C}\right)\right) \cong \mathbb{C}$ is non-zero. It is true, though not trivial, that for this $\mathcal{A}_{\bar{z}}$, it is possible to pick $\mathcal{A}_{z}$ so that the curvature $\mathcal{F}_{z \bar{z}}=\partial_{z} \mathcal{A}_{\bar{z}}-\partial_{\bar{z}} \mathcal{A}_{z}+\left[\mathcal{A}_{z}, \mathcal{A}_{\bar{z}}\right]$ vanishes. One simple fact is that if we write

$$
\mathcal{A}_{z}=\left(\begin{array}{cc}
f & e \\
g & -f
\end{array}\right),
$$

then the upper right matrix element $e$ is a holomorphic function on $C$, which globally must be constant. Looking at the diagonal part of the equation $F_{z \bar{z}}=0$, we learn that $e$ must be nonzero.

Another simple fact is that given any choice of $\mathcal{A}_{z}$ that makes the curvature vanish, any other choice can be obtained by the shift

$$
\mathcal{A}_{z} \rightarrow \mathcal{A}_{z}+\left(\begin{array}{cc}
0 & 0 \\
w & 0
\end{array}\right),
$$

where $w$ is a quadratic differential. So any two opers differ by a quadratic differential. There is actually a canonical way to map the space of opers to the space of quadratic differentials, since the flat $\mathrm{S} L(2, \mathbb{R})$ bundle that comes from uniformization (that is, from 
the existence on $C$ of an Einstein metric of constant negative curvature) can be interpreted as an oper and gives a natural base point in the space of opers.

So for $G=\mathrm{S} U(2)$, the space $\boldsymbol{N}$ of opers is, as a complex manifold, naturally isomorphic to the space $H^{0}\left(C, K_{C}^{2}\right)$ of quadratic differentials on $C$. (If there are marked points on $C$, a similar derivation leads to quadratic differentials that may have a pole of a specified type at the marked point.) For generalized quiver theories [16] associated to $\mathrm{S} U(2), H^{0}\left(C, K_{C}^{2}\right)$ is the same as the base of the Hitchin fibration, which as usual we call $\boldsymbol{B}$. In general, for quiver gauge theories based on any $G, \boldsymbol{N}$ is naturally isomorphic to $\boldsymbol{B}$. (We should point out that there is something strange about this assertion. The natural complex structure on $\boldsymbol{N}$ is obtained by restricting to $\boldsymbol{N}$ the complex structure $J$ on $\mathcal{M}_{H}$, while the natural complex structure on $\boldsymbol{B}$ is similarly related to $I$. Nevertheless, $\boldsymbol{N}$ with its natural complex structure is naturally isomorphic to $\boldsymbol{B}$ with its natural complex structure.)

Though we will not really use this information in the present paper, the reader may find it helpful if we describe how opers are related to conformal field theory. Again, we consider only the case of $\mathrm{S} U(2)$. First of all, locally it is possible to find a gauge transformation of lower triangular form $\left(\begin{array}{ll}1 & 0 \\ * & 1\end{array}\right)$ setting $u$ to zero and otherwise leaving $\mathcal{A}_{\bar{z}}$ in the form (4.16). In such a gauge $\mathcal{A}_{z}$ still has the form (4.17) and $e$ is still a nonzero constant; $f$ and $g$ are now holomorphic sections of $K_{C}$ and $K_{C}^{2}$, respectively. Without changing the form of $\mathcal{A}_{\bar{z}}$, we can make a gauge transformation by a $2 \times 2$ unimodular and holomorphic matrix to set $e=1$ and $f=0$, whence

$$
\mathcal{A}_{z}=\left(\begin{array}{ll}
0 & 1 \\
T & 0
\end{array}\right)
$$

where $T$ is still holomorphic. One might think that $T$ would be a quadratic differential, but actually it is more naturally understood as a stress tensor or projective connection. To see why, consider an infinitesimal gauge transformation generated by

$$
\left(\begin{array}{cc}
\partial_{z} v / 2 & v \\
v T-\partial_{z}^{2} v / 2 & -\partial_{z} v / 2
\end{array}\right),
$$

with $v$ a holomorphic vector field. A short calculation shows that this leaves fixed the form of $\mathcal{A}_{z}$, and that $T$ transforms by

$$
T \rightarrow T+v \partial_{z} T+2\left(\partial_{z} v\right) T-\frac{1}{2} \partial_{z}^{3} v
$$

in other words as a stress tensor.

\subsection{Physical states from the brane of opers}

What we have described so far is the brane $\mathcal{B}_{N}$ that is dual to $\mathcal{B}_{\alpha}$. To find the dual to $\mathcal{B}_{\beta}$, which we call $\mathcal{B}_{N^{\prime}}$, we simply make a $\mathrm{U}(1)_{R}$ rotation. Since $\mathcal{B}_{N}$ is a Lagrangian brane of type $(A, B, A)$, the $\mathrm{U}(1)_{R}$ rotation maps $\mathcal{B}_{N}$ to a Lagrangian brane $\mathcal{B}_{N^{\prime}}$ of type $(A, A, B)$, whose support $\boldsymbol{N}^{\prime}$ is obtained from $\boldsymbol{N}$ by the $\mathrm{U}(1)_{R}$ rotation. Thus $\boldsymbol{N}^{\prime}$ is a rotated version of the brane of opers. 
Our construction gives two related ways to describe the space $\mathcal{H}_{\varepsilon_{1}, \varepsilon_{2}}$ of ground states of the $\mathcal{N}=2$ gauge theory on $\mathbb{R} \times S_{\varepsilon_{1}, \varepsilon_{2}}^{3}$. It is the space of $\left(\mathcal{B}_{\alpha}, \mathcal{B}_{N^{\prime}}\right)$ strings (in a description with gauge group $G, T$-duality on scalars related to the $\alpha$ circle, and coupling parameter $\left.\varepsilon_{1} / \varepsilon_{2}\right)$ or the space of $\left(\mathcal{B}_{N}, \mathcal{B}_{\beta}\right)$ strings (in a description with gauge group $G^{\vee}, T$-duality on scalars related to the $\beta$ circle, and coupling parameter $\left.\varepsilon_{2} / \varepsilon_{1}\right)$.

Either way, this sounds much like what we studied in section 3: one brane is a canonical coisotropic $A$-brane, and the other is a Lagrangian $A$-brane. But there is a crucial difference. Let us consider first the first description. Here $\mathcal{B}_{\alpha}$ is a coisotropic brane of type $(A, B, A)$ with Chan-Paton curvature $F=\omega_{J}$, and $\mathcal{B}_{N^{\prime}}$ is a Lagrangian brane of type $(A, A, B)$. Viewing these as $A$-branes of type $\omega_{I}$, we want to describe the space $\mathcal{H}_{\varepsilon_{1}, \varepsilon_{2}}$ of $\left(\mathcal{B}_{\alpha}, \mathcal{B}_{N^{\prime}}\right)$ strings.

If the Chan-Paton curvature $F$ were nondegenerate when restricted to $\boldsymbol{N}^{\prime}$, then $\mathcal{H}_{\varepsilon_{1}, \varepsilon_{2}}$ would arise by quantization of $N^{\prime}$ in symplectic structure $F$; this was reviewed in section 2.3. Here we are in the opposite situation. Since $F=\omega_{J}$ and $N^{\prime}$ is Lagrangian for $\omega_{J}$, $F$ actually vanishes when restricted to $N^{\prime}$. This situation sounds very special, but actually it is the usual situation considered in geometric Langlands.

In such a case, rather than by quantization, $\mathcal{H}_{\varepsilon_{1}, \varepsilon_{2}}$ can be described as follows. Let $\mathcal{I}=\omega^{-1} F$ be the complex structure determined by the coisotropic brane $\mathcal{B}_{\alpha}$. In the present case, $\omega=\omega_{I}, F=\omega_{J}$, and $\mathcal{I}=K$. Note that $\boldsymbol{N}^{\prime}$ is a complex submanifold in complex structure $K$. Then, roughly speaking, the space of physical states is the space of holomorphic functions on $\boldsymbol{N}^{\prime}$ (actually holomorphic sections of a certain line bundle, as we explain momentarily), in complex structure $K$. Since $\boldsymbol{N}^{\prime}$ is as a complex manifold the same as the base $\boldsymbol{B}$ of the Hitchin fibration, we can think of the space $\mathcal{H}_{\varepsilon_{1}, \varepsilon_{2}}$ of physical states as the space of holomorphic sections of a certain line bundle over $\boldsymbol{B}$.

To describe the relevant line bundle, observe that because of the relation of $D$-branes to $K$-theory, by comparing the Chan-Paton bundles of the two branes in question, one can extract a square root $K_{N^{\prime}}^{1 / 2}$ of the canonical bundle of $N^{\prime} . K_{N^{\prime}}^{1 / 2}$ is a holomorphic line bundle over $\boldsymbol{N}^{\prime}$ that is trivial, but not canonically so. $\mathcal{H}_{\varepsilon_{1}, \varepsilon_{2}}$ is the space of holomorphic sections of $K_{N^{\prime}}^{1 / 2}$.

To justify this answer, we simply quantize open strings that stretch between the two branes $\mathcal{B}_{\alpha}$ and $\mathcal{B}_{N^{\prime}}$. Let the string worldsheet be $\mathbb{R} \times I$ where $\mathbb{R}$ is parametrized by the time $t$, and $I$ is an interval, with boundary conditions at the two ends set by the two branes. To find zero energy states, we quantize the motion in time of the modes that have zero kinetic energy along $I$. We can repeat the derivation in section 2.3 of [14], but the result is now different because $F$ vanishes when restricted to $N^{\prime}$, rather than being nondegenerate. The bosonic zero modes describe maps $x: \mathbb{R} \rightarrow N^{\prime}$. There is no term first order in $\mathrm{d} x / \mathrm{d} t$, because $\left.F\right|_{N^{\prime}}=0$. So the low energy action for $x$ is the usual sort of kinetic energy $\frac{1}{2} \int \mathrm{d} t g_{I J} \frac{\mathrm{d} x^{I}}{\mathrm{~d} t} \frac{\mathrm{d} x^{J}}{\mathrm{~d} t}$, where $g_{I J}$ is the induced metric on $\boldsymbol{N}^{\prime}$. There also are fermionic zero modes $\psi^{I}$, forming a section of the pullback by $x$ of the tangent bundle of $\boldsymbol{N}^{\prime}$. To find this, one can follow eqs. (2.8)-(2.11) of [14], with the difference that now $\boldsymbol{N}^{\prime}$ is real with respect to $J$, rather than holomorphic as assumed in [14]. While a key point in [14] was that there were no fermionic zero modes, now zero modes $\psi^{I}$ do survive. The effective 
action for $x^{I}, \psi^{J}$ must have two supercharges, descending from the unbroken supercharges $Q, \bar{Q}$ of the gauge theory. The minimal supersymmetric action for these fields with this amount of supersymmetry is familiar:

$$
I=\int \mathrm{d} t\left(\frac{1}{2} g_{I J} \frac{\mathrm{d} x^{I}}{\mathrm{~d} t} \frac{\mathrm{d} x^{J}}{\mathrm{~d} t}+i g_{I J} \psi^{I} \frac{D \psi^{J}}{D t}\right) .
$$

This is the basic sigma-model action in one dimension, with two supercharges when (as here) the target space is a Kahler manifold. In quantization, $Q$ becomes the $\bar{\partial}$ operator acting on $(0, q)$-forms with values in $K_{N^{\prime}}^{1 / 2}$, and $\bar{Q}$ is its adjoint. (The sum $\bar{Q}+Q$ is the Dirac operator.) The cohomology of $Q$ is thus the $\bar{\partial}$ cohomology of $N^{\prime}$ with values in $K_{N^{\prime}}^{1 / 2}$. The cohomology of degree 0 consists of holomorphic sections of $K_{N^{\prime}}^{1 / 2}$, and the higher cohomology vanishes.

So finally, upon identifying $\boldsymbol{N}^{\prime}$ as a complex manifold with $\boldsymbol{B}$, the base of the Hitchin fibration, the space $\mathcal{H}_{\varepsilon_{1}, \varepsilon_{2}}$ of physical states can be identified as the space of holomorphic sections of $K_{B}^{1 / 2}$ over $B$ :

$$
\mathcal{H}_{\varepsilon_{1}, \varepsilon_{2}}=H^{0}\left(\boldsymbol{B}, K_{B}^{1 / 2}\right) .
$$

There is a slight surprise here: the right hand side does not depend on the parameters $\varepsilon_{1}, \varepsilon_{2}$. However, the action of observables on $\mathcal{H}_{\varepsilon_{1}, \varepsilon_{2}}$ does depend on these parameters, as we will see shortly.

We obtained this result starting from the interpretation of $\mathcal{H}_{\varepsilon_{1}, \varepsilon_{2}}$ as the space of $\left(\mathcal{B}_{\alpha}, \mathcal{B}_{N^{\prime}}\right)$ strings. The alternative realization of $\mathcal{H}_{\varepsilon_{1}, \varepsilon_{2}}$ as the space of $\left(\mathcal{B}_{N}, \mathcal{B}_{\beta}\right)$ strings would lead after analogous steps to a different identification of $\mathcal{H}_{\varepsilon_{1}, \varepsilon_{2}}$ with $H^{0}\left(\boldsymbol{B}, K_{\boldsymbol{B}}^{1 / 2}\right)$.

As in somewhat similar situations considered in section 2.3 of [14], the gauge theory on $\mathbb{R} \times S_{\alpha, \beta}^{3}$, or the two-dimensional sigma-model on $\mathbb{R} \times I$ to which it reduces, will generate a hermitian inner product on $\mathcal{H}_{\varepsilon_{1}, \varepsilon_{2}}=H^{0}\left(\boldsymbol{B}, K_{\boldsymbol{B}}^{1 / 2}\right)$ making this space a Hilbert space. But, except in the limit of large $\varepsilon_{1} / \varepsilon_{2}$, when the Kahler modulus of $\mathcal{M}_{H}$ becomes large and the two-dimensional $\sigma$-model can be treated semiclassically, this hermitian inner product is not necessarily given by any elementary classical formula.

\subsection{Observables}

Now we want to describe the algebra of observables that acts on the space $\mathcal{H}_{\varepsilon_{1}, \varepsilon_{2}}$ of ground states of the gauge theory on $S_{\varepsilon_{1}, \varepsilon_{2}}^{3}$. In the brane description, $\mathcal{H}_{\varepsilon_{1}, \varepsilon_{2}}$ is the space of $\left(\mathcal{B}_{\alpha}, \mathcal{B}_{N}\right)$ strings or alternatively the space of $\left(\mathcal{B}_{N^{\prime}}, \mathcal{B}_{\beta}\right)$ strings. The first description makes it clear that the algebra of $\left(\mathcal{B}_{\alpha}, \mathcal{B}_{\alpha}\right)$ strings acts on $\mathcal{H}_{\varepsilon_{1}, \varepsilon_{2}}$ by attaching to the left end of a string. The second description makes it equally clear that the algebra of $\left(\mathcal{B}_{\beta}, \mathcal{B}_{\beta}\right)$ strings acts on $\mathcal{H}_{\varepsilon_{1}, \varepsilon_{2}}$ by attaching to the right end of a string. The strings in question are the physical states in the $A$-model of type $\omega_{I}$.

The two algebras of $\left(\mathcal{B}_{\alpha}, \mathcal{B}_{\alpha}\right)$ strings and $\left(\mathcal{B}_{\beta}, \mathcal{B}_{\beta}\right)$ strings are equivalent, up to the usual steps $\left(\mathrm{a} \mathrm{U}(1)_{R}\right.$ rotation that may act on mass parameters, an exchange $\varepsilon_{1} \leftrightarrow \varepsilon_{2}$, and duality $\left.G \leftrightarrow G^{\vee}\right)$. So let us just describe the algebra of $\left(\mathcal{B}_{\beta}, \mathcal{B}_{\beta}\right)$ strings.

The brane $\mathcal{B}_{\beta}$ has Chan-Paton curvature $F=\left(\varepsilon_{1} / \varepsilon_{2}\right) \omega_{K}$, and we study it in the $A$-model of symplectic structure $\omega=\left(\varepsilon_{1} / \varepsilon_{2}\right) \omega_{I}$. The $\left(\mathcal{B}_{\beta}, \mathcal{B}_{\beta}\right)$ strings are obtained by 
deformation quantization of the ring $\mathcal{R}$ of holomorphic functions in complex structure $\mathcal{I}=\omega^{-1} F=J$. To proceed farther, we focus on the case of a generalized quiver theory associated to a Riemann surface $C$. The two-dimensional description involves a sigmamodel with target $\mathcal{M}_{H}$, and in complex structure $J, \mathcal{M}_{H}$ is the moduli space of $\mathfrak{g}_{\mathbb{C}}$-valued flat connections $\mathcal{A}$ on $C$. The ring of holomorphic functions on $\mathcal{M}_{H}$ is generated by traces of holonomies. ${ }^{14}$ In other words, for $\gamma \subset C$ a simple closed curve and $R$ a representation of $G$, we consider the function

$$
W_{R}(\gamma)=\operatorname{Tr}_{R} P \exp \left(-\oint_{\gamma} \mathcal{A}\right)
$$

such functions generate $\mathcal{R}$.

The deformation of the commutative ring $\mathcal{R}$ to a noncommutative but still associative ring depends on one complex parameter, usually called $q$. This deformation, which is constructed in [51] (of course there are also many related constructions from different points of view), appears in two familiar quantum field theory problems. One is the problem [52] of quantizing Chern-Simons gauge theory with compact gauge group $G$ on $\mathbb{R} \times C$, where $C$ is a Riemann surface. The phase space is $\mathcal{M}$, the moduli space of flat unitary (that is $\mathfrak{g}$-valued) connections $A$ on $C$. The basic operators on the physical Hilbert space are Wilson loop operators. These are the same traces of holonomies considered in the last paragraph, except that they are functions on $\mathcal{M}$, the space of flat $\mathfrak{g}$-valued connections, rather than its complexification $\mathcal{M}_{H}$. However, traces of holonomies regarded as functions on $\mathcal{M}$ have natural analytic continuations to holomorphic functions on $\mathcal{M}_{H}$. (The analytic continuations are given by the same traces, now evaluated for flat connections that may be complex-valued.) So the ring of classical observables of Chern-Simons theory with compact gauge group $G$ is actually the same as the ring $\mathcal{R}$ of complex holonomies considered in the last paragraph. In quantizing Chern-Simons gauge theory with gauge group $G, \mathcal{R}$ is deformed to a noncommutative, associative algebra of quantum Wilson loop operators. The deformation parameter is the Chern-Simons level $k$, in terms of which one defines $q=\exp (2 \pi i /(k+h))$, where $h$ is the dual Coxeter number of $G$. A physical Hilbert space on which the quantum algebra can act exists only for positive integer $k$. But the deformed algebra $\mathcal{R}_{q}$ of Wilson loop operators can be constructed as a function of a complex variable $q$. The procedure involved can be understood as deformation quantization (for example, see [53] for this interpretation). But the situation is much better than a typical example of deformation quantization: this is a favorable case in which deformation quantization gives a deformation parametrized by a complex variable $q$, not just a deformation over a formal power series ring.

The second related problem in which one encounters the deformed ring $\mathcal{R}_{q}$ of holonomy functions is two-dimensional conformal field theory. The most basic case is current algebra

\footnotetext{
${ }^{14}$ This fact is not obvious but is proved for classical groups in [49], with the understanding that by "holomorphic functions" on $\mathcal{M}_{H}$, we really mean algebraic functions on $\mathcal{M}_{H}$, viewed as a moduli space of representations of the fundamental group of the Riemann surface $C$. Despite the fact that traces of holonomies suffice to generate the ring, it may be more natural to consider also the functions associated to labeled graphs, as for example in [50].
} 
of a compact group $G$. Here a monodromy operation on the space of conformal blocks was introduced in [54] (and this influenced subsequent work on Chern-Simons theory). The monodromy operation is defined by transporting a primary field in a representation $R$ of $G$ around a loop $\gamma \subset C$. In the correspondence between two-dimensional conformal field theory and three-dimensional Chern-Simons theory, the space of conformal blocks maps to the physical Hilbert space of Chern-Simons theory, and the conformal field theory monodromies map to the action of Wilson loop operators of gauge theory.

The conformal field theory operation just mentioned has been adapted to Liouville theory in $[55,56]$. The deformation parameter is the Liouville coupling $b$, which corresponds to the Chern-Simons level $k$ if Liouville theory is related to $\mathrm{S} L(2, \mathbb{R})$ current algebra. So the same associative algebra $\mathcal{R}_{q}$ of quantized holonomies that can be extracted from $\mathrm{S} U(2)$ Chern-Simons theory acts on the conformal blocks of Liouville theory. This algebra is also seen in quantization of Teichmuller space. For gauge groups of higher rank, there is a similar relation between the quantum-deformed algebra of holonomies in Chern-Simons gauge theory with compact gauge group, and the deformed algebra of holonomies that acts in the quantization of the higher rank analogs of Teichmuller space [46, 47].

There is, however, a fundamental difference between the case of a compact symmetry group such as $\mathrm{S} U(2)$ and the case of a noncompact group such as $\mathrm{S} L(2, \mathbb{R})$. In the case of a compact symmetry group or gauge group $G$, the deformed algebra $\mathcal{R}_{q}$ of holonomies acts irreducibly on the space of conformal blocks of two-dimensional conformal field theory, or equivalently the Hilbert space of Chern-Simons theory. For Liouville theory or $\mathrm{S} L(2, \mathbb{R})$ Chern-Simons theory, this is far from being true. Instead, in its action on the space of Virasoro conformal blocks, the algebra $\mathcal{R}_{q}$ commutes with a dual algebra $\mathcal{R}_{q^{\prime}}$. $\mathcal{R}_{q^{\prime}}$ is a second deformed algebra of holonomy operators, with its parameter differing by the Liouville duality $b \leftrightarrow b^{-1}$.

In our presentation here, the action of the second commuting algebra is manifest, since the $\left(\mathcal{B}_{\alpha}, \mathcal{B}_{\alpha}\right)$ strings acting at one end commute with the $\left(\mathcal{B}_{\beta}, \mathcal{B}_{\beta}\right)$ strings acting at the other end.

The space of Virasoro conformal blocks can be characterized as an irreducible module for two algebras $\mathcal{R}_{q}$ and $\mathcal{R}_{q^{\prime}}$ of $\mathrm{S} L(2, \mathbb{R})$ holonomies at dual values of the parameters. The two algebras are noncommutative but associative, and commute with each other. The fact that we have found this structure strongly supports the idea that the Hilbert space $\mathcal{H}_{\varepsilon_{1}, \varepsilon_{2}}$ of the generalized quiver theory on $S_{\varepsilon_{1}, \varepsilon_{2}}^{3}$ is indeed the space of Virasoro (or Liouville) conformal blocks, as first argued in [15].

\subsubsection{Wilson and 't Hooft operators}

Concretely, the algebras $\mathcal{R}_{q}$ and $\mathcal{R}_{q^{\prime}}$ are generated by $Q$-invariant Wilson and 't Hooft operators that act at one end of $S_{\alpha, \beta}^{3}$ or the other. Let us return to the explicit description (4.1) of $S_{\alpha, \beta}^{3}$. When we toroidally reduce $S_{\alpha, \beta}^{3}$ to an interval $I$ parametrized by $w$ with $0 \leq w \leq \ell$, the end at $w=0$ is really a circle $S_{\beta}^{1}$ parametrized by $\beta$, and similarly the end at $w=\ell$ is a circle $S_{\alpha}^{1}$ parametrized by $\alpha$. The rings $\mathcal{R}_{q}$ and $\mathcal{R}_{q^{\prime}}$ are generated by supersymmetric loop operators wrapped on $S_{\beta}^{1}$ or $S_{\alpha}^{1}$ at a fixed time. 
In generalized quiver gauge theories associated to a Riemann surface $C$, half-BPS Wilson and 't Hooft operators are in one-to-one correspondence [57] with data of the following kind: a choice of a homotopy class of simple closed loop $\gamma \subset C$ together with a choice of a representation $R$ of $G$. Of course, this construction is usually made in undeformed super Yang-Mills theory. However, near either end of $S_{\alpha, \beta}^{3}$, the vector field that we have used to make the $\Omega$-deformation reduces to either $\epsilon_{2} \partial / \partial \beta$ (at $w=0$ ) or $\epsilon_{1} \partial / \partial \alpha($ at $w=\ell$ ). So we can rotate from the ordinary theory to the $\Omega$-deformed theory as in section 3.2, and hence the half-BPS operators studied in [57] have analogs in our situation. The holonomy functions (4.24) that generate $\mathcal{R}_{q}$ and $\mathcal{R}_{q}^{\prime}$ simply come from the corresponding half-BPS loop operators.

It was already observed in [15] that half-BPS loop operators can be wrapped on the circles $S_{\alpha}^{1}$ and $S_{\beta}^{1}$ and that in the $\Omega$-deformed theory, these operators do not commute. What we have contributed is to relate this fact to the noncommutativity that arises in two-dimensional $\sigma$-models with a $B$-field, and to formulate the problem in a way that is closer to other occurrences of the noncommutative ring $\mathcal{R}_{q}$ in mathematical physics.

\subsubsection{Winding states of the $A$-model}

We now make a slight digression, aiming to spare the reader some puzzlement by briefly answering the following question. Given the $T$-duality between $\mathcal{B}_{\beta}$ and $\mathcal{B}_{N}$, the space of $\left(\mathcal{B}_{\beta}, \mathcal{B}_{\beta}\right)$ strings must be equivalent to the space of $\left(\mathcal{B}_{N}, \mathcal{B}_{N}\right)$ strings. But $\mathcal{B}_{N}$ is an ordinary Lagrangian $A$-brane, so the space of $\left(\mathcal{B}_{N}, \mathcal{B}_{N}\right)$ strings in the $A$-model, as usually understood, is simply the cohomology of $N$. How can we possibly identify the space of $\left(\mathcal{B}_{N}, \mathcal{B}_{N}\right)$ strings with a ring of holonomy functions?

The answer to this question is that it is necessary to take into account something that is usually not relevant - winding states in the $A$-model. As a prototype of the problem, we consider a sigma-model with target $W=\mathbb{R} \times S^{1}$, with the obvious product metric and with coordinates $t, \theta$ on $\mathbb{R}$ and $S^{1}$. First we consider the $B$-model on $W$, in the obvious complex structure in which $t+i \theta$ is holomorphic. Let $\mathcal{B}$ be a $B$-brane whose support is all of $W$, with trivial Chan-Paton bundle. The $(\mathcal{B}, \mathcal{B})$ strings of zero ghost number are associated to the holomorphic functions

$$
f_{n}=\exp (n(t+i \theta)), \quad n \in \mathbb{Z}
$$

We note that apart from $f_{0}$, which is the constant function 1 , all other $f_{n}$ have non-zero momentum around $S^{1}$, and exponential growth along $\mathbb{R}$ in one direction or the other.

Now we perform $T$-duality along the $S^{1}$ direction. This maps the $B$-model of $W$ to an $A$-model of $\widetilde{W}=\mathbb{R} \times \widetilde{S}^{1}$, where $\widetilde{S}^{1}$ is the dual circle. The brane $\mathcal{B}$ is mapped to a Lagrangian $A$-brane $\mathcal{B}^{\prime}$ that is supported on $\mathbb{R} \times p$, with $p$ a point in $\widetilde{S}^{1}$. The $(\mathcal{B}, \mathcal{B})$ string corresponding to the identity function $f_{0}$ maps to the $\left(\mathcal{B}^{\prime}, \mathcal{B}^{\prime}\right)$ string that corresponds to the zero-dimensional cohomology of $\mathbb{R} \times p$. What about the $f_{n}$ with $n \neq 0$ ? As they carry momentum along $S^{1}$, they correspond to $A$-model states that have winding around $\widetilde{S}^{1}$. Of course, these states also have exponential growth along $\mathbb{R}$, since this property is unaffected by $T$-duality. The reason that such $A$-model winding states are unfamiliar is that we do not usually study $A$-model states with exponential growth. 
To see that this example really is a prototype for the original question, note that the product of two copies of $W$ is $X=\mathbb{R}^{2} \times T^{2}$, which we can think of as the moduli space of vacua for the familiar example of a free vector multiplet. The product of two copies of the brane $\mathcal{B}$ considered above is the space-filling brane $\mathcal{B}_{*}$ of type $(B, B, B)$ on $X$. Turning on a Chan-Paton curvature proportional to $\omega_{J}$, this can be deformed to a coisotropic $A$-brane of type $(A, B, A)$, without changing the essentials of the above discussion. Thus, even for a free vector multiplet, we have to go beyond the usual class of $A$-model states to see the duality between the spaces of $\left(\mathcal{B}_{\beta}, \mathcal{B}_{\beta}\right)$ strings and of $\left(\mathcal{B}_{N}, \mathcal{B}_{N}\right)$ strings.

\subsection{Partition functions}

The formulation in [15] was actually slightly different from what we have given here and focused on partition functions. The main claims there were that the partition function of a generalized quiver theory on $\mathbb{R}_{\varepsilon_{1}, \varepsilon_{2}}^{4}$ gives a chiral conformal block in Liouville theory, and that the partition function of such a theory on $S_{\varepsilon_{1}, \varepsilon_{2}}^{4}$ (at least for $\epsilon_{1}=\epsilon_{2}$ ) gives the modularinvariant partition function of Liouville theory with left- and right-movers included.

It makes sense to compare the various spaces that are involved here, because $\mathbb{R} \times S^{3}$ is equivalent topologically to $\mathbb{R}^{4}$ with a point at the origin omitted, while $S^{4}$ can be viewed as $\mathbb{R}^{4}$ with a point at infinity added. Comparing the three spaces in this way, the $\mathrm{U}(1) \times \mathrm{U}(1)$ action that we have used to make an $\Omega$-deformation on $\mathbb{R} \times S^{3}$ can be extended over $\mathbb{R}^{4}$ or $S^{4}$. Then we make the $\Omega$-deformation on all three spaces with the same parameters $\varepsilon_{1}, \varepsilon_{2}$, and we refer to the result as a generalized quiver theory on $\mathbb{R} \times S_{\varepsilon_{1}, \varepsilon_{2}}^{3}, \mathbb{R}_{\varepsilon_{1}, \varepsilon_{2}}^{4}$, or $S_{\varepsilon_{1}, \varepsilon_{2}}^{4}$.

In general, in any four-dimensional quantum field theory, let $B$ be a four-manifold with boundary $S^{3}$. Then the path integral on $B$ gives a physical state in the Hilbert space associated to $S^{3}$. In the context of topologically twisted four-dimensional gauge theory, we have to make a choice, as was remarked in section 4.1: on $\mathbb{R} \times S^{3}$, topological twisting conserves two supercharges, $Q$ and its adjoint $\bar{Q}$, but only one can be conserved on a more general four-manifold $B$. Let us make a choice and conserve $Q$. If in addition the $\mathrm{U}(1) \times \mathrm{U}(1)$ action of $S^{3}$ extends over $B$, we can make the $\Omega$-deformation on $B$ and in that case, the path integral on $B$ will give a vector in the $Q$-cohomology of the $\Omega$-deformed theory on $S^{3}$, in other words, a Virasoro (or Liouville) conformal block.

The most simple choice of $B$ that has the right properties is a four-dimensional ball $B^{4}$, as explained at the end of section 4.1. Writing $B_{\varepsilon_{1}, \varepsilon_{2}}^{4}$ for the $\Omega$-deformed version of $B^{4}$, the path integral over $B_{\varepsilon_{1}, \varepsilon_{2}}^{4}$ will give a Virasoro conformal block $\mathcal{W}$. Observing that $B_{\varepsilon_{1}, \varepsilon_{2}}^{4}$ is the same topologically as $\mathbb{R}_{\varepsilon_{1}, \varepsilon_{2}}^{4}$, and that this equivalence is compatible with the $\mathrm{U}(1) \times \mathrm{U}(1)$ action, what we have just said is equivalent to the "chiral" version of the claim in [15].

For the non-chiral version of their claim, we want to glue together two copies of $B_{\varepsilon_{1}, \varepsilon_{2}}^{4}$, with opposite orientation, along their common boundary $S_{\varepsilon_{1}, \varepsilon_{2}}^{3}$. If we preserve the same topological supercharge in both copies of $B_{\varepsilon_{1}, \varepsilon_{2}}^{4}$, we will get Donaldson theory on $S_{\varepsilon_{1}, \varepsilon_{2}}^{4}$. This is not what we want. Instead, to get the claim of [15], we must preserve one supercharge $Q$ on one copy of $B_{\varepsilon_{1}, \varepsilon_{2}}^{4}$ and its conjugate $\bar{Q}$ on the other copy. Then, the partition function on $S_{\varepsilon_{1}, \varepsilon_{2}}^{4}$ gives the norm squared of the Virasoro conformal block $\mathcal{W}$, in the Hilbert space $\mathcal{H}_{\varepsilon_{1}, \varepsilon_{2}}$. 
In general, it is not clear how to make opposite topological twists in the two hemispheres of $S_{\varepsilon_{1}, \varepsilon_{2}}^{4}$. For $\epsilon_{1}=\epsilon_{2}=1 / R$, it has been shown [58] that the $\Omega$-deformed theory with opposite twists on the two sides is equivalent to physical Yang-Mills theory on a foursphere of radius $R$, with no $\Omega$-deformation at all. This fact was exploited in [15]. We hope that this fact can be adapted for our derivation and has a useful generalization to other values of the deformation parameters. Until this is found, we may fall back on the approach of [59] in two dimensions. In that approach, between the two copies of $B_{\varepsilon_{1}, \varepsilon_{2}}^{4}$, one places a very long cylinder $I \times S_{\varepsilon_{1}, \varepsilon_{2}}^{3}$. In the limit that the length of $I$ becomes much greater than the radius of $S_{\varepsilon_{1}, \varepsilon_{2}}^{3}$, the path integral on $I \times S_{\varepsilon_{1}, \varepsilon_{2}}^{3}$ projects onto quantum ground states, and the path integral on $S_{\varepsilon_{1}, \varepsilon_{2}}^{4}$ can be evaluated in the space $\mathcal{H}_{\varepsilon_{1}, \varepsilon_{2}}$ of quantum ground states even though we make opposite topological twists at the two ends.

\subsection{Flatness}

One basic fact about conformal field theory on a Riemann surface $C$ is that the space of conformal blocks is locally independent of the complex structure of $C$. There is a projectively flat connection that can be used to transport the space of conformal blocks as the complex structure of $C$ varies. This holds for theories with finite-dimensional spaces of conformal blocks as well as for more sophisticated theories such as Liouville theory. See [60] for more discussion.

In our present context, this implies that although the definition of the variety $\boldsymbol{N}$ of opers depends on the complex structure of $C$, the space $H^{0}\left(\boldsymbol{N}, K_{\boldsymbol{N}}^{1 / 2}\right)$ does not. More precisely, the bundle of Hilbert spaces over the moduli space of complex structures on $C$ whose fiber is $H^{0}\left(\boldsymbol{N}, K_{N}^{1 / 2}\right)$ should admit a projectively flat connection. In principle, the $\sigma$-model must generate this connection but we do not know what sort of semiclassical formula can be given for it.

One may ask whether the underlying $(0,2)$ model in six dimensions predicts the existence of this flat connection. The $R$-symmetry group of this theory is $\mathrm{SO}(5)_{R}$. There is no non-trivial homomorphism from $\mathrm{S} O(6)$ to $\mathrm{S} O(5)$, so the $(0,2)$ model does not have a twisted version that would lead to a topological field theory in six dimensions. However, suppose that we specialize to six-manifolds of the form $\mathbb{R} \times W$, with a product metric; here $W$ is a five-manifold. The structure group of the tangent bundle of $W$ is $\mathrm{SO}(5)$, which of course does admit an isomorphism with $\mathrm{S} O(5)_{R}$. So a twisted theory can be constructed for six-manifolds of the from $\mathbb{R} \times W$. This theory has a supercharge $Q$ obeying $Q^{2}=0$, and it is plausible that the cohomology of $Q$ is locally independent of the metric of $W$.

This is close to what we want. What we have been studying is an $\Omega$-deformed version of the cohomology of the twisted theory just described for the case $W=S^{3} \times C$. Of course, we did not introduce the subject in precisely this way. Rather we started with a fourdimensional generalized quiver theory, which can be obtained from the $(0,2)$ theory in six dimensions by compactifying on $C$ with a topological twist that preserves supersymmetry. Then we compactified on $S^{3}$, again with a topological twist that preserves supersymmetry. The net effect was to compactify from six dimensions to one dimension on $W=S^{3} \times C$ with a topological twist that preserves supersymmetry. There is essentially only one way to do this - the twist mentioned in the last paragraph that preserves five-dimensional symmetry. 
This seems promising. The only catch is that it is not clear how to include the $\Omega$ deformation in this analysis, since one does not have a good understanding of the $\Omega$ deformation in six-dimensional terms. To explain the existence of a flat connection on the bundle of Hilbert spaces $\mathcal{H}_{\varepsilon_{1}, \varepsilon_{2}}$ over the Teichmuller space of $C$, we obviously need an argument that takes the $\Omega$-deformation into account.

\subsection{Including surface operators}

The correspondence between gauge theory and two-dimensional conformal field theory becomes richer $[61,62]$ if surface operators are included. Here we consider a half-BPS surface operator inserted at one of the two ends of $S_{\alpha, \beta}^{3}$. Near the support of the surface operator, the spacetime looks like $\Sigma \times D$ where $\Sigma$ is a two-manifold and $D$ is the usual cigar geometry. The surface operator is inserted at $\Sigma \times p$, where $p$ is the tip of the cigar. In our applications, $\Sigma=\mathbb{R} \times S^{1}$. The $\Omega$ deformation is made using a vector field that generates the rotation of $D$ around $p$ (section 3 ) or one that also acts at the same time by rotation of $S^{1}$ (the present section). Far from the tip, $D$ looks like a cylinder $\mathbb{R} \times \widetilde{S}^{1}$.

A half-BPS surface operator inserted on $\Sigma \times p$ preserves all supersymmetry that is compatible with the $\Omega$-deformation (or with the curvature of $D$ ), so it can be included naturally in our analysis. First we consider the abelian case $G=\mathrm{U}(1)$.

We consider the simplest half-BPS surface operator. It is defined by introducing a Lie algebra valued parameter $\alpha$ and requiring that the curvature have a delta-function singularity $F / 2 \pi \sim \alpha \delta_{\Sigma \times p}$. Equivalently, the holonomy around the singularity is $\exp (2 \pi i \alpha)$.

Now let us consider the two-dimensional sigma-model, with target $\mathcal{M}_{H}$, that we get by compactification on $S^{1} \times \widetilde{S}^{1}$. The tip of $D$ together with the surface operator will give a brane in this model. In the absence of the surface operator, we observed that supersymmetry requires that the curvature along $D$ vanishes. Hence the holonomy around $\widetilde{S}^{1}$ is trivial, and the circle-valued field that we called $A_{3}$ in section 3 vanishes at the boundary. To get a more useful description, in section 3.1.1, we $T$-dualized this field to another angle-valued field $\varrho$. Vanishing of $A_{3}$ meant that $\varrho$ obeys Neumann boundary conditions. All other bosonic fields in the sigma-model obey Neumann boundary conditions for more obvious reasons. So altogether, the brane $\mathcal{B}$ that comes from the tip of the cigar has all of $\mathcal{M}_{H}$ for its support. Of course, this is familiar from section 3.1.1.

Including the surface operator changes this analysis in only one way. Vanishing of curvature along $D$ now requires that the holonomy around $\widetilde{S}^{1}$ should equal $\exp (2 \pi i \alpha)$ (rather than 1) or equivalently that the boundary value of $A_{3}$ is equal to $\alpha$. After $T$ duality, the dual field $\varrho$ still obeys Neumann boundary conditions, but the circle that it parametrizes is equipped with a flat Chan-Paton line bundle $\mathcal{S}$. The sole effect of the surface operator on the brane $\mathcal{B}$ that comes from the tip of the cigar is that the ChanPaton bundle of that brane is tensored by $\mathcal{S}$. There is no problem in describing $\mathcal{S}$ explicitly as a line bundle over $\mathcal{M}_{H}$. For $G=\mathrm{U}(1), \mathcal{M}_{H}=\mathbb{R}^{2} \times T^{2}$ and $\mathcal{S}$ is simply a flat line bundle with holonomy around one of the directions in $T^{2}$. As $\mathcal{S}$ is flat, tensoring with $\mathcal{S}$ preserves all supersymmetry.

Now let us consider the nonabelian case. We will consider the case of a generalized quiver theory [16] based on $\mathrm{S} U(2)$, but we start with the case of a single $\mathrm{S} U(2)$ gauge 
group. The parameter $\alpha$ now takes values in the Lie algebra of a maximal torus of $\mathrm{SU}(2)$. There is really only one major change in the above analysis. To describe it, let us work over a generic part of the base $\boldsymbol{B}$ of the Hitchin fibration where $\mathrm{SU}(2)$ is broken to an abelian subgroup, isomorphic to $\mathrm{U}(1)$. Then the monodromy around $\widetilde{S}^{1}$ is again $\mathrm{U}(1)$ valued, computed from the zero mode of an effective scalar field that we will again call $A_{3}$. And $A_{3}$ is again $T$-dualized to another scalar $\varrho$ to go to a more useful description. There is only one major difference from the case of $G=\mathrm{U}(1)$. Vanishing of the curvature along $D$, instead of requiring $A_{3}$ and $\alpha$ to be equal (as circle-valued objects), now requires only that they should be equal up to a Weyl transformation. Thus (for $G=\mathrm{S} U(2)$ ), there are now two allowed boundary values of $A_{3}$, namely $A_{3}= \pm \alpha$.

After $T$-duality, each of the allowed boundary values of $A_{3}$ maps to a flat line bundle over the circle parametrized by $\varrho$. As $A_{3}$ has two allowed boundary values, this gives a rank two flat bundle over the circle. As the other fields vary, this will give a rank two bundle $\mathcal{U} \rightarrow \mathcal{M}_{H}$ (at least in the region where our approximations apply). The effect of the surface operator is to tensor the Chan-Paton bundle of the brane $\mathcal{B}$ that comes from the tip of the cigar with $\mathcal{U}$.

Our description so far has been based on an approximation valid far from the discriminant locus in $\boldsymbol{B}$. In this approximation, $\mathcal{U}$ is a flat vector bundle. It is not possible for this to be the whole story, since the total space of the Seiberg-Witten or Hitchin fibration for any gauge theory with a semi-simple gauge group has a finite fundamental group. The most optimistic hypothesis is that the full description of the surface operator involves a rank two bundle $\mathcal{U} \rightarrow \mathcal{M}_{H}$ that is flat far from the discriminant locus and in general preserves all supersymmetry. We will assume this to be the case, though it is also imaginable that something more complicated happens near the discriminant locus in $\boldsymbol{B}$.

Before making a proposal for a more concrete description of $\mathcal{U}$, we will consider $\mathrm{S} U(2)$ generalized quiver theories that arise from six dimensions by compactification on a Riemann surface $C$. The gauge group is a product of $\mathrm{S} U(2)$ factors. According to [61], in such a theory, there is a natural family of surface operators parametrized by the choice of a point $q \in C$. In suitable local descriptions, an operator in this family can be described precisely as above with the relevant part of the gauge group being simply $\mathrm{S} U(2)$. Hence the above analysis applies, and the insertion at the tip of the cigar of a surface operator associated to a point $q \in C$ will generate a rank two bundle $\mathcal{U}_{q} \rightarrow \mathcal{M}_{H}$.

As $\mathcal{U}_{q}$ varies in $C$, the $\mathcal{U}_{q}$ will fit together as fibers of a rank two holomorphic vector bundle $\mathcal{U} \rightarrow \mathcal{M}_{H} \times C$. There is an obvious candidate for what this bundle may be: the universal bundle, or more exactly, the bundle part of the universal Higgs bundle. In other words, every point $r \in \mathcal{M}_{H}$ parametrizes a solution of Hitchin's equations - a holomorphic bundle $E \rightarrow C$ together with a Higgs field $\varphi \in H^{0}\left(C, \operatorname{ad}(E) \otimes K_{C}\right)$ and a hermitian metric such that Hitchin's equations are satisfied. (Here we emphasized a holomorphic point of view, but of course there are many equivalent descriptions of this data.) The universal Higgs bundle is a triple consisting of a holomorphic bundle $\mathcal{E} \rightarrow \mathcal{M}_{H} \times C$, a Higgs field $\widehat{\varphi}: \mathcal{E} \rightarrow \mathcal{E} \otimes K_{C}$, and a hermitian metric on $\mathcal{E}$, such that the restriction of this data to $r \times C$, for any $r \in \mathcal{M}_{H}$, is the solution of Hitchin's equations corresponding to $r$. (For an elementary description of universal bundles, including subtleties involving the center of the 
gauge group, see section 7.1 of [13].) By restricting $\mathcal{E}$ to $\mathcal{M}_{H} \times q$, we get a very plausible candidate for $\mathcal{U}_{q}$.

In the absence of the $\Omega$ deformation, the condition that tensoring with $\mathcal{U}_{q}$ preserves all supersymmetry means that $\mathcal{U}_{q}$ must be holomorphic in every complex structure on $\mathcal{M}_{H}$. This follows from standard properties of the universal Higgs bundle. Upon making the $\Omega$ deformation, further properties are needed, and they are not well-understood, since the theory of coisotropic $A$-branes of rank greater than 1 has not been developed. Possibly the necessary properties follow from the existence and properties of the universal Higgs field $\widehat{\varphi}$.

\section{Acknowledgments}

Part of this work has been done while NN visited the IAS and while EW visited the IHES and the SCGP. We thank these institutes for their hospitality. Research of NN was partly supported by l'Agence Nationale de la Recherche under grants ANR-06-BLAN-3_137168 and ANR-05-BLAN-0029-01, and by the Russian Foundation for Basic Research through the grants RFBR N 09-02-00393 and NSh-3036.2008.2. Research of EW was partly supported by NSF Grant Phy-0503584. We thank R. Donagi, E. Frenkel, D. Gaiotto, S. Gukov, G. Moore, S. Shatashvili, J. Teschner, and Y. Tachikawa for comments and discussions.

\section{A The general $\Omega$-deformation in toroidal compactification}

\section{A.1 The setup}

The purpose of this appendix is to generalize various computations involving compactification on a two-torus which in the text were done in special cases. Here we will allow an arbitrary flat metric on the two-torus, not necessarily rectangular, and we allow arbitrary $\epsilon$ parameters for the $\Omega$-deformation. Since we focus on the two-torus, we will simplify somewhat the notation used in the text.

We denote the torus as $T_{\circ}$ and endow it with a constant metric $G_{I J}, I, J=1,2$ :

$$
\mathrm{d} s_{T_{\circ}}^{2}=G_{i j} \mathrm{~d} \theta^{i} \mathrm{~d} \theta^{j}, \quad \theta^{i} \sim \theta^{i}+2 \pi .
$$

The gauge theory probes the dual torus $T_{\circ} \vee$, the moduli space of flat $\mathcal{U}(1)$-connections on $T_{\circ}$. We write such a connection as

$$
A=i \alpha_{1} \mathrm{~d} \theta^{1}+i \alpha_{2} \mathrm{~d} \theta^{2}
$$

with constant hermitian matrices $\alpha_{1}, \alpha_{2}$ (our connection form $A$ is anti-hermitian). The gauge transformations generated by the $\mathcal{U}(1)$-valued functions

$$
u_{n_{1}, n_{2}}=\exp \left(i n_{1} \theta^{1}+i n_{2} \theta^{2}\right)
$$

shift the components $\alpha_{1,2}$ by $n_{1,2}$, respectively. The natural metric on $T_{\circ}^{\vee}$ is given by:

$$
\mathrm{d} s_{T_{\circ} \vee}^{2}=\frac{1}{(2 \pi i)^{2}} \int_{T_{\circ}} \mathrm{d} A \wedge \star \mathrm{d} A=\sqrt{\operatorname{det}(G)} G^{i j} \mathrm{~d} \alpha_{i} \mathrm{~d} \alpha_{j} .
$$

It depends only on the complex structure of $T_{\circ}$. 
It is convenient to parametrize $G^{i j}$ by two complex numbers $\omega_{1,2}$,

$$
G^{i j} \mathrm{~d} \alpha_{i} \mathrm{~d} \alpha_{j}=\left|\omega_{1} \mathrm{~d} \alpha_{1}+\omega_{2} \mathrm{~d} \alpha_{2}\right|^{2}
$$

defined up to a simultaneous phase rotation, so that the invariants are:

$$
\begin{aligned}
\left|\omega_{1}\right|^{2} & =G^{11}, \\
\left|\omega_{2}\right|^{2} & =G^{22}, \\
\operatorname{Re}\left(\omega_{1} \bar{\omega}_{2}\right) & =G^{12} .
\end{aligned}
$$

Let us assume

$$
\operatorname{Im}\left(\frac{\omega_{2}}{\omega_{1}}\right)>0
$$

We then have:

$$
\sqrt{\operatorname{det}(G)}=\frac{1}{\operatorname{Im}\left(\omega_{2} \bar{\omega}_{1}\right)} \text {. }
$$

For a rectangular torus,

$$
\omega_{1}=\frac{1}{\rho_{1}}, \quad \omega_{2}=\frac{i}{\rho_{2}}
$$

Let us first consider the case of gauge group $\mathcal{U}(1)$. We take the Maxwell action to be

$$
I=\frac{1}{8 \pi} \int_{X^{4}} \mathrm{~d}^{4} x \sqrt{g}\left(\frac{4 \pi}{\mathrm{g}_{4}^{2}} F_{m n} F^{m n}+\frac{i \vartheta}{4 \pi} \epsilon_{m n p q} F^{m n} F^{p q}\right) .
$$

If we take the four-manifold to be $\Sigma \times T_{\circ}$, with the product metric $h \times G$, with $h$ being the metric on $\Sigma$, and denote the Riemannian measure of $\Sigma$ as d $\mu$, then, in the low-energy approximation, (A.8) reads as:

$$
\begin{aligned}
I & =\frac{(2 \pi)^{2}}{8 \pi} \sqrt{\operatorname{det}(G)} \int_{\Sigma} \mathrm{d} \mu\left(\frac{8 \pi}{\mathrm{g}_{4}^{2}} h^{a b} G^{i j}\left(\partial_{a} A_{i} \partial_{b} A_{j}\right)-\frac{i \vartheta}{\pi} \epsilon^{a b} \epsilon^{i j}\left(\partial_{a} A_{i} \partial_{b} A_{j}\right)\right) \\
& =-i \vartheta \int_{\Sigma} \mathrm{d} \alpha_{1} \wedge \mathrm{d} \alpha_{2}+\frac{4 \pi^{2} \sqrt{\operatorname{det}(G)}}{\mathrm{g}_{4}^{2}} \int_{\Sigma} \mathrm{d}\left(\omega_{1} \alpha_{1}+\omega_{2} \alpha_{2}\right) \wedge \star \mathrm{d}\left(\bar{\omega}_{1} \alpha_{1}+\bar{\omega}_{2} \alpha_{2}\right) .
\end{aligned}
$$

\section{A.2 The familiar story}

We study the pure $\mathcal{N}=2$ gauge theory with the simple gauge group $G$, its maximal torus $\mathbf{T}$ and Cartan subalgebra $\mathfrak{t}=\operatorname{Lie} \mathbf{T}$, and with a gauge coupling $g_{4}$ and theta angle $\vartheta$. The complex scalar in the vector multiplet is denoted by $\phi$.

In compactifying this theory on $T_{0}$, in the small radius or low energy approximation, one can impose the constraints

$$
[\phi, \bar{\phi}]=0, \quad\left[A_{1}, A_{2}\right]=0,
$$

and assume everything is independent of the angular coordinates $\theta^{1}, \theta^{2}$.

Thus

$$
A_{1}=i \alpha_{1}, \quad A_{2}=i \alpha_{2},
$$

where from now on $\alpha_{1,2} \in(\mathfrak{t} \otimes \mathbb{R}) /\left(\Lambda_{\text {cwt }} \otimes \mathbb{Z}\right)$, with $\Lambda_{\text {cwt }}$ the coweight lattice of $G$. One must divide by the Weyl group acting diagonally on $\alpha_{1,2}$ as well as $\phi$. 


\section{A.2.1 The bosonic lagrangian}

The bosonic part of the pure $\mathcal{N}=2$ gauge theory Lagrangian reduced on the torus $T_{\circ}$ is given at low energies by

$$
\begin{aligned}
& L=\frac{8 \pi^{2}}{2 \mathrm{~g}_{4}^{2}} \sqrt{\operatorname{det}(G)} \operatorname{tr}\left\{\left(\omega_{1} \mathrm{~d} \alpha_{1}+\omega_{2} \mathrm{~d} \alpha_{2}\right) \wedge \star\left(\bar{\omega}_{1} \mathrm{~d} \alpha_{1}+\bar{\omega}_{2} \mathrm{~d} \alpha_{2}\right)+\mathrm{d} \phi \wedge \star \mathrm{d} \bar{\phi}\right\} \\
& -i \vartheta \operatorname{tr} \mathrm{d} \alpha_{1} \wedge \mathrm{d} \alpha_{2},
\end{aligned}
$$

where "tr" denotes the induced metric on $\mathfrak{t}$. The gauge theory part of this Lagrangian can be borrowed from (A.9).

We view here $\alpha_{1,2} \in \mathfrak{t} \otimes \mathbb{R} /\left(\Lambda_{\text {cwt }} \otimes \mathbb{Z}\right)$ as real, and $\phi \in \mathfrak{t} \otimes \mathbb{C}$ as complex, with "tr" defining a positive definite inner product on $\mathfrak{t}$. The Euclidean path integral measure is given by

$$
e^{-\int L}
$$

The condition for a field configuration to be invariant under the supercharge $Q$ that is relevant to Donaldson theory and the $\Omega$-deformation is

$$
\mathrm{d} \phi=0, \quad \bar{\partial}\left(\omega_{1} \alpha_{1}+\omega_{2} \alpha_{2}\right)=0,
$$

where the second equation is anti-selfduality of the gauge field in our low energy approximation. For such fields, (A.13) evaluates to:

$$
\exp \left(-\int L\right)=\exp \left(2 \pi i \tau_{0} \int \operatorname{tr} \mathrm{d} \alpha_{1} \wedge \mathrm{d} \alpha_{2}\right),
$$

where the complexified gauge coupling is equal to

$$
\tau_{0}=\frac{\vartheta}{2 \pi}+\frac{4 \pi i}{\mathrm{~g}_{4}^{2}}
$$

\section{A.2.2 T-duality to $\mathcal{M}_{H}$}

In what follows, we use the notation

$$
\mu_{0}=\frac{8 \pi^{2}}{\mathrm{~g}_{4}^{2}} \sqrt{\operatorname{det}(G)}=2 \pi \frac{\operatorname{Im}\left(\tau_{0}\right)}{\operatorname{Im}\left(\omega_{2} \bar{\omega}_{1}\right)}
$$

The Lagrangian (A.12) describes a sigma-model with target the product of a torus and $\mathfrak{t} \otimes \mathbb{C}$, all divided by the Weyl group. Upon $T$-duality along the $\alpha_{1}$ direction, we map it to a sigma-model on $\mathcal{M}_{H}$, after taking into account the nonlinear corrections.

The $T$-duality is performed in the standard fashion. The first step is to replace $\mathrm{d} \alpha_{1}$ in (A.12) by an independent $\mathfrak{t}$-valued one-form $p_{1}$ and add the term $-2 \pi i \operatorname{tr}\left(p_{1} \wedge \mathrm{d} \widetilde{\alpha}_{1}\right)$ to $L$, with the understanding that $\widetilde{\alpha}_{1}$ takes values in a circle of circumference 1 :

$$
\begin{aligned}
L^{\prime}= & \frac{\mu_{0}}{2} \operatorname{tr}\left\{\left(\omega_{1} p_{1}+\omega_{2} \mathrm{~d} \alpha_{2}\right) \wedge \star\left(\bar{\omega}_{1} p_{1}+\bar{\omega}_{2} \mathrm{~d} \alpha_{2}\right)+\mathrm{d} \phi \wedge \star \mathrm{d} \bar{\phi}\right\} \\
& +2 \pi i \operatorname{tr}\left\{\left(\mathrm{d} \widetilde{\alpha}_{1}+\frac{\vartheta}{2 \pi} \mathrm{d} \alpha_{2}\right) \wedge p_{1}\right\}
\end{aligned}
$$


Integrating over $\widetilde{\alpha}_{1}$ would lead us back to (A.12). Instead we integrate over $p_{1}$. The path integral over $p_{1}$ is Gaussian, with the saddle point for $p_{1}$ at:

$$
p_{1}=-\operatorname{Re}\left(\frac{\omega_{2}}{\omega_{1}}\right) \mathrm{d} \alpha_{2}+i \star \frac{2 \pi}{\mu_{0}\left|\omega_{1}\right|^{2}}\left(\mathrm{~d} \widetilde{\alpha}_{1}+\frac{\vartheta}{2 \pi} \mathrm{d} \alpha_{2}\right) .
$$

In terms of the left- and right-moving components of $\alpha_{1}$, (A.19) reads as follows:

$$
\begin{aligned}
\alpha_{1}^{L} & =\frac{2 \pi}{\mu_{0}\left|\omega_{1}\right|^{2}}\left(\widetilde{\alpha}_{1}+\frac{\vartheta}{2 \pi} \alpha_{2}\right)-\operatorname{Re}\left(\frac{\omega_{2}}{\omega_{1}}\right) \alpha_{2} \\
\alpha_{1}^{R} & =-\frac{2 \pi}{\mu_{0}\left|\omega_{1}\right|^{2}}\left(\widetilde{\alpha}_{1}+\frac{\vartheta}{2 \pi} \alpha_{2}\right)-\operatorname{Re}\left(\frac{\omega_{2}}{\omega_{1}}\right) \alpha_{2} .
\end{aligned}
$$

The $T$-dual Lagrangian is given by:

$$
\begin{aligned}
L^{T}= & \frac{\mu_{0}}{2}\left\{\operatorname{tr} \frac{\left|\operatorname{Im}\left(\omega_{2} \bar{\omega}_{1}\right)\right|^{2}}{\left|\omega_{1}\right|^{2}} \mathrm{~d} \alpha_{2} \wedge \star \mathrm{d} \alpha_{2}+\mathrm{d} \phi \wedge \star \mathrm{d} \phi+(2 \pi)^{2} \frac{\left(\mathrm{d} \widetilde{\alpha}_{1}+\frac{\vartheta}{2 \pi} \mathrm{d} \alpha_{2}\right) \wedge \star\left(\mathrm{d} \widetilde{\alpha}_{1}+\frac{\vartheta}{2 \pi} \mathrm{d} \alpha_{2}\right)}{\mu_{0}^{2}\left|\omega_{1}\right|^{2}}\right\} \\
& -2 \pi i \operatorname{Re}\left(\frac{\omega_{2}}{\omega_{2}}\right) \operatorname{tr}\left(\mathrm{d} \widetilde{\alpha}_{2}+\frac{\vartheta}{2 \pi} \mathrm{d} \alpha_{2}\right) \wedge \mathrm{d} \alpha_{2} .
\end{aligned}
$$

Introduce the $\mathfrak{t} \otimes \mathbb{C}$-valued dimensionless coordinates $\mathcal{Z}, \mathcal{W}$ :

$$
\begin{aligned}
\mathcal{Z} & =\widetilde{\alpha}_{1}+\frac{\vartheta}{2 \pi} \alpha_{2}+\frac{4 \pi i}{\mathrm{~g}_{4}^{2}} \alpha_{2} \\
\mathcal{W} & =\frac{1}{2 \pi} \mu_{0} \bar{\omega}_{1} \phi .
\end{aligned}
$$

The choice of phase in the definition of $\mathcal{W}$ is a matter of convenience. Most of the time we assume $\omega_{1}=\left|\omega_{1}\right| \in \mathbb{R}_{+}$anyway. In terms of $\mathcal{W}$ and $\mathcal{Z}$, eq. (A.21) takes the form:

$$
\begin{aligned}
L^{T}=\frac{(2 \pi)^{2}}{2 \mu_{0}\left|\omega_{1}\right|^{2}} \operatorname{tr}\{\mathrm{d} \mathcal{Z} \wedge & \star \mathrm{d} \overline{\mathcal{Z}}+\mathrm{d} \mathcal{W} \wedge \star \mathrm{d} \overline{\mathcal{W}}\} \\
& -2 \pi i \operatorname{Re}\left(\frac{\omega_{2}}{\omega_{1}}\right) \operatorname{tr}\left(\mathrm{d} \widetilde{\alpha}_{1}+\frac{\vartheta}{2 \pi} \mathrm{d} \alpha_{2}\right) \wedge \mathrm{d} \alpha_{2} .
\end{aligned}
$$

Note that

$$
\frac{2 \pi^{2}}{\mu_{0}\left|\omega_{1}\right|^{2}}=\pi \frac{\operatorname{Im}\left(\frac{\omega_{2}}{\omega_{1}}\right)}{\operatorname{Im}\left(\tau_{0}\right)}
$$

We deduce from (A.23) the target space metric

$$
\mathrm{d} s_{\mathcal{M}_{H}}^{2}=2 \pi \frac{\operatorname{Im}\left(\omega_{2} / \omega_{1}\right)}{\operatorname{Im}\left(\tau_{0}\right)}(\mathrm{d} \mathcal{Z} \mathrm{d} \overline{\mathcal{Z}}+\mathrm{d} \mathcal{W} \mathrm{d} \overline{\mathcal{W}}) .
$$

In our approximation, the target space metric is flat; in the exact theory, it is a complete hyper-Kahler metric on what we usually call $\mathcal{M}_{H}$. We also deduce from (A.23) a $B$-field, which, up to exact terms, is given by:

$$
B=\frac{2 \pi}{2 i} \frac{\operatorname{Re}\left(\omega_{2} / \omega_{1}\right)}{\operatorname{Im}\left(\tau_{0}\right)}(\mathrm{d} \mathcal{Z} \wedge \mathrm{d} \overline{\mathcal{Z}}+\mathrm{d} \mathcal{W} \wedge \mathrm{d} \overline{\mathcal{W}})=\left(\operatorname{Re}\left(\omega_{2} / \omega_{1}\right)\right) \omega_{I}
$$


Here $\omega_{I}$ is the topologically normalized symplectic form on $\mathcal{M}_{H}$, which is Kähler in the complex structure $I$. The functions of $\mathcal{Z}, \mathcal{W}$ are holomorphic in complex structure $I$.

As we explained in section 3.1.2, the supersymmetry of Donaldson theory or the $\Omega$-deformation is that of the $B$-model in complex structure $I$. Indeed, the equations (A.14) read:

$$
\begin{gathered}
\bar{\partial}\left(\omega_{1} \alpha_{1}^{L}+\omega_{2} \alpha_{2}\right)=0 \leftrightarrow \\
\omega_{1} \bar{\partial}\left(\frac{2 \pi}{\mu_{0}\left|\omega_{1}\right|^{2}}\left(\widetilde{\alpha}_{1}+\frac{\vartheta}{2 \pi} \alpha_{2}\right)+i \operatorname{Im}\left(\omega_{2} / \omega_{1}\right) \alpha_{2}\right)=0 \leftrightarrow \bar{\partial} \mathcal{Z}=0 \\
\partial\left(\bar{\omega}_{1} \alpha_{1}^{R}+\bar{\omega}_{2} \alpha_{2}\right)=0 \leftrightarrow \\
\bar{\omega}_{1} \partial\left(-\frac{2 \pi}{\mu_{0}\left|\omega_{1}\right|^{2}}\left(\widetilde{\alpha}_{1}+\frac{\vartheta}{2 \pi} \alpha_{2}\right)-i \operatorname{Im}\left(\omega_{2} / \omega_{1}\right) \alpha_{2}\right)=0 . \leftrightarrow \partial \mathcal{Z}=0
\end{gathered}
$$

Here we used (A.25) and:

$$
\omega_{2}-\operatorname{Re}\left(\omega_{2} / \omega_{1}\right) \omega_{1}=i \omega_{1} \operatorname{Im}\left(\omega_{2} / \omega_{1}\right)
$$

This, together with the obvious $\mathrm{d} \phi=0 \leftrightarrow \mathrm{d} \mathcal{W}=0$ implies

$$
\mathrm{d} \mathcal{W}=0, \quad \mathrm{~d} \mathcal{Z}=0 .
$$

To summarize, the sigma-model that comes from compactification on a two-torus has $\mathcal{M}_{H}$ as its target space and the usual supercharge is the one associated to the $B$-model of type $I$. Let us write $\omega_{I}^{*}$ for the Kahler form of $\mathcal{M}_{H}$ in complex structure $I$. Then we have

$$
\omega_{I}^{*}=\operatorname{Im}\left(\omega_{2} / \omega_{1}\right) \omega_{I}, B=\operatorname{Re}\left(\omega_{2} / \omega_{1}\right) \omega_{I} .
$$

The complexified gauge coupling $\tau_{0}$ maps to the complex structure of the (asymptotic) fiber of Hitchin's fibration. This is to be compared to the alternative approach [13] to the same class of models by compactification of $\mathcal{N}=4$ super Yang-Mills theory from four to two dimensions on a Riemann surface. Not surprisingly, the rôle of the complexified gauge coupling of $\mathcal{N}=4$ super Yang-Mills is played in the present approach by $\omega_{2} / \omega_{1}$ :

$$
\tau_{\mathcal{N}=4}=\frac{\omega_{2}}{\omega_{1}} .
$$

\section{A.3 The $\Omega$-deformation}

Now let us turn on the $\Omega$-deformation, with the parameters $\epsilon_{1}, \epsilon_{2}$, corresponding to the shifts along the $\theta^{1}, \theta^{2}$ directions. The Lagrangian becomes

$$
\begin{aligned}
\frac{\mu_{0}}{2} \operatorname{tr} & \left\{\left(\omega_{1} \mathrm{~d} \alpha_{1}+\omega_{2} \mathrm{~d} \alpha_{2}\right) \wedge \star\left(\bar{\omega}_{1} \mathrm{~d} \alpha_{1}+\bar{\omega}_{2} \mathrm{~d} \alpha_{2}\right)\right\} \\
& +\frac{\mu_{0}}{2} \operatorname{tr}\left\{\left(\mathrm{d} \phi+\epsilon_{1} \mathrm{~d} \alpha_{1}+\epsilon_{2} \mathrm{~d} \alpha_{2}\right) \wedge \star\left(\mathrm{d} \bar{\phi}+\bar{\epsilon}_{1} \mathrm{~d} \alpha_{1}+\bar{\epsilon}_{2} \mathrm{~d} \alpha_{2}\right)\right\} \\
& -i \vartheta \operatorname{tr} \mathrm{d} \alpha_{1} \wedge \mathrm{d} \alpha_{2} .
\end{aligned}
$$


It is convenient to rewrite this formula using quaternions. Let $\mathbf{i}, \mathbf{j}, \mathbf{k}$ denote the usual quaternion imaginary units,

$$
\mathbf{i j}=\mathbf{k}, \mathbf{i}^{2}=\mathbf{j}^{2}=\mathbf{k}^{2}=-1, \mathbf{i j}=-\mathbf{j i}, \ldots
$$

The elements of $\mathbb{H}=\mathbb{R} \oplus \mathbb{R} \mathbf{i} \oplus \mathbb{R} \mathbf{j} \oplus \mathbb{R} \mathbf{k}$ can be conveniently represented as $z+w \mathbf{j}, z, w \in$ $\mathbb{C}=\mathbb{R} \oplus \mathbb{R} \mathbf{i}$. We have the trace map Re $: \mathbb{H} \rightarrow \mathbb{R}$,

$$
\operatorname{Re}\left(x_{0}+x_{1} \mathbf{i}+x_{2} \mathbf{j}+x_{3} \mathbf{k}\right)=x_{0}, \operatorname{Re}(\mathbf{a b})=\operatorname{Re}(\mathbf{b a}),
$$

and the conjugation map:

$$
\left(x_{0}+x_{1} \mathbf{i}+x_{2} \mathbf{j}+x_{3} \mathbf{k}\right)^{\dagger}=\left(x_{0}-x_{1} \mathbf{i}-x_{2} \mathbf{j}-x_{3} \mathbf{k}\right)
$$

Introduce the following $\mathfrak{t} \otimes \mathbb{H}$ valued field:

$$
\mathbf{X}=\omega_{1} \alpha_{1}+\omega_{2} \alpha_{2}+\left(\phi+\epsilon_{1} \alpha_{1}+\epsilon_{2} \alpha_{2}\right) \mathbf{j}
$$

Recall that we view $\alpha_{1}, \alpha_{2}$ as real variables, i.e. they commute with the quaternions. On the other hand, $\phi$ obeys:

$$
\phi \mathbf{i}=\mathbf{i} \phi, \quad \phi \mathbf{j}=\mathbf{j} \bar{\phi}, \quad \phi \mathbf{k}=\mathbf{k} \bar{\phi}
$$

The conjugated field is

$$
\mathbf{X}^{\dagger}=\bar{\omega}_{1} \alpha_{1}+\bar{\omega}_{2} \alpha_{2}-\mathbf{j}\left(\bar{\phi}+\bar{\epsilon}_{1} \alpha_{1}+\bar{\epsilon}_{2} \alpha_{2}\right) .
$$

The action (A.32) reads, simply:

$$
L=\frac{\mu_{0}}{2} \operatorname{Re}\left(\operatorname{tr}\left(\mathrm{d} \mathbf{X} \wedge \star \mathrm{d} \mathbf{X}^{\dagger}\right)\right)-i \vartheta \operatorname{tr} \mathrm{d} \alpha_{1} \wedge d \alpha_{2} .
$$

The advantage of our definition of $\mathbf{X}$ was to remove explicit deformation parameters from the Lagrangian. We recognize in (A.36) the Lagrangian of a sigma-model on $(\mathfrak{t} \otimes \mathbb{H}) /\left(\Lambda_{\text {cwt }} \otimes\right.$ $\Gamma$ ) where now $\Gamma \cong \mathbb{Z} \oplus \mathbb{Z}$ is the two-dimensional lattice embedded in $\mathbb{H} \approx \mathbb{C} \oplus \mathbb{C} \mathbf{j}$ as follows:

$$
\Gamma=\mathbb{Z} \varpi_{1}+\mathbb{Z} \varpi_{2}
$$

where

$$
\varpi_{1}=\omega_{1}+\epsilon_{1} \mathbf{j}, \quad \varpi_{2}=\omega_{2}+\epsilon_{2} \mathbf{j} .
$$

A remark on relative phases. In deciding to combine $\phi$ and $\alpha_{1,2}$ to $\mathbf{X}$ as in (A.34) and $\omega_{1,2}$ and $\epsilon_{1,2}$ as in (A.38), we made a choice of a relative phase of $\epsilon_{1,2}$ and $\omega_{1,2}$. This choice has no intrinsic meaning, as long as we consider a theory with $\mathcal{U}(1)_{R}$ symmetry. In fact, there are two arbitrary phases; one can be fixed by requiring $\omega_{1}=\left|\omega_{1}\right|$, while the other involves the phases of the deformation parameters. The appearance of these phases is related to the impossibility to decide canonically, within an $\mathcal{N}=2$ gauge theory that has $\mathcal{U}(1)_{R}$ symmetry, which complex structure on $\mathcal{M}_{H}$ is $J$ and which is $K$ (of course, once $J$ is agreed upon, $K=I J$ follows). In general, the distinction between $J$ and $K$ becomes meaningful upon incorporation of mass parameters that break $\mathcal{U}(1)_{R}$ symmetry. However, we will not incorporate such parameters here. 


\section{A.3.1 The supersymmetry of the $\Omega$-deformed theory}

The supercharge which is favored by the $\Omega$-background enforces the following field equations:

$$
\begin{aligned}
\mathrm{d}\left(\phi+\epsilon_{1} \alpha_{1}+\epsilon_{2} \alpha_{2}\right) & =0 \\
\bar{\partial}\left(\omega_{1} \alpha_{1}+\omega_{2} \alpha_{2}\right) & =0 \\
\partial\left(\bar{\omega}_{1} \alpha_{1}+\bar{\omega}_{2} \alpha_{2}\right) & =0 .
\end{aligned}
$$

Upon performing $T$-duality along the $\alpha_{1}$-direction, the equations (A.39) become:

$$
\begin{aligned}
& \bar{\partial} W^{+}=\bar{\partial} Z^{+}=0, \\
& \partial W^{-}=\partial Z^{-}=0,
\end{aligned}
$$

with

$$
\begin{aligned}
W^{+} & =\left(\phi+\epsilon_{1} \alpha_{1}+\epsilon_{2} \alpha_{2}\right)^{L} \\
Z^{+} & =\left(\omega_{1} \alpha_{1}+\omega_{2} \alpha_{2}\right)^{L} \\
W^{-} & =\left(\phi+\epsilon_{1} \alpha_{1}+\epsilon_{2} \alpha_{2}\right)^{R} \\
Z^{-} & =\left(-\bar{\omega}_{1} \alpha_{1}-\bar{\omega}_{2} \alpha_{2}\right)^{R} .
\end{aligned}
$$

The minus sign in the definition of $Z^{-}$is chosen for convenience. We need to express $W^{ \pm}, Z^{ \pm}$in terms of $\widetilde{\alpha}_{1}, \alpha_{2}, \phi, \bar{\phi}$, and relate them to the geometry of $\mathcal{M}_{H}$. We shall find that $W^{ \pm}, Z^{ \pm}$are the holomorphic coordinates in two distinct complex structures $\mathcal{I}^{ \pm}$on $\mathcal{M}_{H}$.

\section{A.3.2 Undoing the $\Omega$-deformation}

We can find two unit quaternions $g_{ \pm} \in \mathrm{S} U(2), g_{ \pm}=\mathbf{a}_{ \pm}+\mathbf{b}_{ \pm} \mathbf{j},\left|\mathbf{a}_{ \pm}\right|^{2}+\left|\mathbf{b}_{ \pm}\right|^{2}=1, g_{+} g_{+}^{\dagger}=$ $g_{-} g_{-}^{\dagger}=1$, such that

$$
\begin{aligned}
& \Omega_{1}=g_{+}^{\dagger}\left(\omega_{1}+\epsilon_{1} \mathbf{j}\right) g_{-} \\
& \Omega_{2}=g_{+}^{\dagger}\left(\omega_{2}+\epsilon_{2} \mathbf{j}\right) g_{-}
\end{aligned}
$$

for two complex numbers $\Omega_{1}, \Omega_{2}$. (To accomplish this, pick $g_{+}^{\dagger}$ to diagonalize the quaternion $\left(\omega_{1}+\epsilon_{1} \mathbf{j}\right)\left(\bar{\omega}_{2}-\epsilon_{2} \mathbf{j}\right)$, and similarly choose $g_{-}^{\dagger}$ to diagonalize $\left(\bar{\omega}_{2}-\epsilon_{2} \mathbf{j}\right)\left(\omega_{1}+\epsilon_{1} \mathbf{j}\right)$. To "diagonalize" a quaternion means to conjugate it into $\mathbb{C}$.)

Some easy consequences of (A.42) are

$$
\begin{aligned}
\left|\Omega_{1}\right|^{2} & =\left|\omega_{1}\right|^{2}+\left|\epsilon_{1}\right|^{2}, \quad\left|\Omega_{2}\right|^{2}=\left|\omega_{2}\right|^{2}+\left|\epsilon_{2}\right|^{2} \\
\operatorname{Re}\left(\Omega_{1} \bar{\Omega}_{2}\right) & =\operatorname{Re}\left(\omega_{1} \bar{\omega}_{2}+\epsilon_{1} \bar{\epsilon}_{2}\right) \\
\bar{\Omega}_{1} & =g_{-}^{\dagger}\left(\bar{\omega}_{1}-\epsilon_{1} \mathbf{j}\right) g_{+}, \quad \bar{\Omega}_{2}=g_{-}^{\dagger}\left(\bar{\omega}_{2}-\epsilon_{2} \mathbf{j}\right) g_{+} \\
g_{+} \Omega_{1} g_{-}^{\dagger} & =\omega_{1}+\epsilon_{1} \mathbf{j}, \quad g_{+} \Omega_{2} g_{-}^{\dagger}=\omega_{2}+\epsilon_{2} \mathbf{j} .
\end{aligned}
$$

Again, we have some phase ambiguities. The periods $\Omega_{1,2}$ can be simultaneously rotated, i.e. multiplied by a phase. This is equivalent to the right multiplication of $g_{-}$and $g_{+}$by arbitrary phases:

$$
g_{ \pm} \mapsto g_{ \pm} e^{i \alpha_{ \pm}}
$$


We can, as usual, normalize $\Omega_{1,2}$ in such a way that:

$$
\Omega_{1}=\left|\Omega_{1}\right|, \quad \operatorname{Im} \Omega_{2}>0 .
$$

This reduces (A.44) down to a single phase ambiguity:

$$
\left(g_{+}, g_{-}\right) \mapsto\left(g_{+} e^{i \alpha}, g_{-} e^{i \alpha}\right) .
$$

\section{A.3.3 Rotating the fields}

The Lagrangian (A.36) is unchanged in form if $\mathbf{X}$ replaced by $\widetilde{\mathbf{X}}$,

$$
\widetilde{\mathbf{X}}=g_{+}^{\dagger} \mathbf{X} g_{-}=\Omega_{1} \alpha_{1}+\Omega_{2} \alpha_{2}+\phi_{\|}+\phi_{\perp} \mathbf{j},
$$

where

$$
\phi_{\|}+\phi_{\perp} \mathbf{j}=g_{+}^{\dagger} \phi \mathbf{j} g_{-} .
$$

One can express $\phi_{\|}$as

$$
\phi_{\|}=\varphi_{1} \Omega_{1}+\varphi_{2} \Omega_{2},
$$

where $\varphi_{1,2} \in \mathfrak{t}$ are real components that can be deduced from

$$
\begin{aligned}
\operatorname{Re}\left(\phi_{\|} \bar{\Omega}_{2}\right) & =\operatorname{Re}\left(\phi \bar{\epsilon}_{2}\right) \\
\operatorname{Re}\left(\phi_{\|} \bar{\Omega}_{1}\right) & =\operatorname{Re}\left(\phi \bar{\epsilon}_{1}\right) \\
\bar{\phi}_{\|}-\phi_{\perp} \mathbf{j} & =-g_{-}^{\dagger} \phi \mathbf{j} g_{+} .
\end{aligned}
$$

We will not need to compute these components explicitly.

The Lagrangian (A.36) becomes

$$
\begin{aligned}
& \frac{\mu_{0}}{2} \operatorname{tr}\left\{\left(\mathrm{d} \phi_{\|}+\Omega_{1} \mathrm{~d} \alpha_{1}+\Omega_{2} \mathrm{~d} \alpha_{2}\right) \wedge \star\left(\mathrm{d} \bar{\phi}_{\|}+\bar{\Omega}_{1} \mathrm{~d} \alpha_{1}+\bar{\Omega}_{2} \mathrm{~d} \alpha_{2}\right)+\mathrm{d} \phi_{\perp} \wedge \star \mathrm{d} \bar{\phi}_{\perp}\right\} \\
& \quad-i \vartheta \operatorname{tr} \mathrm{d} \alpha_{1} \wedge \mathrm{d} \alpha_{2} .
\end{aligned}
$$

By shifting $\alpha_{1,2}$ by $-\varphi_{1,2}$, we could eliminate $\phi_{\|}$at the expense of adding exact terms to the $B$-field.

\section{A.3.4 T-duality, again}

We now $T$-dualize $\alpha_{1}$, as in section A.2. The steps are the same; after a replacement $\mathrm{d} \alpha_{1} \mapsto p_{1}$, we shift $L \rightarrow L^{\prime}=L_{\mathrm{d} \alpha_{1} \mapsto p_{1}}-2 \pi i \operatorname{tr} p_{1} \wedge \mathrm{d} \widetilde{\alpha}_{1}$, and then integrate out $p_{1}$.

Since (up to a trivial $B$-field shift), (A.51) looks like (A.12) with $\omega_{1,2}$ replaced by $\Omega_{1,2}$ (and no change in $\mu_{0}$ ), we must end up with the sigma-model on $\mathcal{M}_{H}$ with the complexified Kahler class

$$
\tau_{\mathcal{N}=4}=\frac{\Omega_{2}}{\Omega_{1}}
$$

and the asymptotic complex structure of the fibers given by:

$$
\tau=\frac{\vartheta}{2 \pi}+\frac{4 \pi i}{\mathrm{~g}_{4}^{2}} \frac{\operatorname{Im}\left(\Omega_{2} \bar{\Omega}_{1}\right)}{\operatorname{Im}\left(\omega_{2} \bar{\omega}_{1}\right)} .
$$


One can compute the right hand side of (A.53), using (A.43), but we shall not need these formulas.

Let us now perform the steps of the $T$-duality more explicitly. The on-shell value of $p_{1}$ is given by:

$$
p_{1}=-\operatorname{dRe}\left(\frac{\phi_{\|}}{\Omega_{1}}\right)-\operatorname{Re}\left(\frac{\Omega_{2}}{\Omega_{1}}\right) \mathrm{d} \alpha_{2}+2 \pi i \star \frac{\mathrm{d} \widetilde{\alpha}_{1}+\frac{\vartheta}{2 \pi} \mathrm{d} \alpha_{2}}{\mu_{0}\left|\Omega_{1}\right|^{2}} .
$$

Note that here and below $\mu_{0}$ is given by (A.17), with $\omega_{1,2}$, not $\Omega_{1,2}$. In terms of the leftand right-moving components of $\alpha_{1}^{L, R},(\mathrm{~A} .54)$ reads:

$$
\begin{aligned}
\alpha_{1}^{L} & =\frac{2 \pi}{\mu_{0}\left|\Omega_{1}\right|^{2}}\left(\widetilde{\alpha}_{1}+\frac{\vartheta}{2 \pi} \alpha_{2}\right)-\operatorname{Re}\left(\frac{\phi_{\|}}{\Omega_{1}}\right)-\operatorname{Re}\left(\frac{\Omega_{2}}{\Omega_{1}}\right) \alpha_{2} \\
\alpha_{1}^{R} & =-\frac{2 \pi}{\mu_{0}\left|\Omega_{1}\right|^{2}}\left(\widetilde{\alpha}_{1}+\frac{\vartheta}{2 \pi} \alpha_{2}\right)-\operatorname{Re}\left(\frac{\phi_{\|}}{\Omega_{1}}\right)-\operatorname{Re}\left(\frac{\Omega_{2}}{\Omega_{1}}\right) \alpha_{2} .
\end{aligned}
$$

Having eliminated $p_{1}$ from $L^{\prime}$ we arrive at (we write $|\mathrm{d} x|^{2}$ instead of $\mathrm{d} x \wedge \star \mathrm{d} \bar{x}$ to avoid clutter):

$$
\begin{aligned}
L^{T}= & \frac{\mu_{0}}{2} \operatorname{tr}\left\{\left|\mathrm{d} \phi_{\perp}\right|^{2}+\left|\Omega_{1}\right|^{2}\left|\mathrm{~d}\left(\operatorname{Im}\left(\frac{\phi_{\|}}{\Omega_{1}}\right)+\operatorname{Im}\left(\frac{\Omega_{2}}{\Omega_{1}}\right) \alpha_{2}\right)\right|^{2}\right\}+\frac{(2 \pi)^{2}}{2 \mu_{0}\left|\Omega_{1}\right|^{2}}\left|\mathrm{~d}\left(\widetilde{\alpha}_{1}+\frac{\vartheta}{2 \pi} \alpha_{2}\right)\right|^{2} \\
& +2 \pi i \mathrm{~d}\left(\operatorname{Re}\left(\frac{\phi_{\|}}{\Omega_{1}}\right)+\operatorname{Re}\left(\frac{\Omega_{2}}{\Omega_{1}}\right) \alpha_{2}\right) \wedge \mathrm{d}\left(\widetilde{\alpha}_{1}+\frac{\vartheta}{2 \pi} \alpha_{2}\right) .
\end{aligned}
$$

We now introduce the complex coordinates $\mathcal{Z}, \mathcal{W}$ on the target space of the effective sigma-model:

$$
\begin{aligned}
\mathcal{Z} & =\widetilde{\alpha}_{1}+\frac{\vartheta}{2 \pi} \alpha_{2}+\frac{i \mu_{0}}{2 \pi}\left(\operatorname{Im}\left(\Omega_{2} \bar{\Omega}_{1}\right) \alpha_{2}+\operatorname{Im}\left(\phi_{\|} \bar{\Omega}_{1}\right)\right) \\
\mathcal{W} & =\frac{1}{2 \pi} \mu_{0} \bar{\Omega}_{1} \phi_{\perp} .
\end{aligned}
$$

In terms of $\mathcal{Z}, \mathcal{W}$ the Lagrangian (A.56) reads simply:

$$
L^{T}=\frac{(2 \pi)^{2}}{2 \mu_{0}\left|\Omega_{1}\right|^{2}} \operatorname{tr}\left(|\mathrm{d} \mathcal{Z}|^{2}+|\mathrm{d} \mathcal{W}|^{2}\right)-i \operatorname{Re}\left(\Omega_{2} / \Omega_{1}\right) \omega_{I}
$$

up to exact terms in the $B$-field. Note that $\mathcal{Z}=\widetilde{\alpha}_{1}+\tau \alpha_{2}$ up to a shift by $\operatorname{Im}\left(\phi_{\|} \bar{\Omega}_{1}\right)$, with $\tau$ given in (A.53). This shows that the fibers of Hitchin's fibration indeed have the complex structure determined by $\tau$, not $\tau_{0}$.

\section{A.4 Rotated complex structures}

Now comes the hard work - the determination of the left- and right-moving complex structures $\mathcal{I}^{ \pm}$relevant to the $T$-dualized theory, and of the Kahler class of the effective $A$-model. 


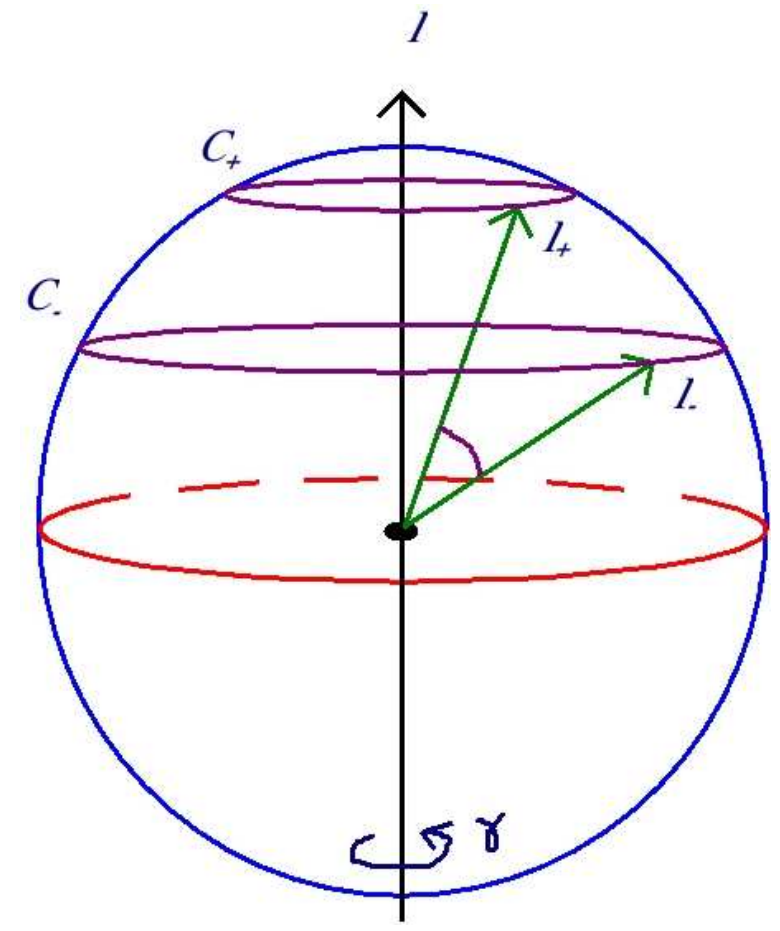

Figure 1. The complex structures $\mathcal{I}^{ \pm}, I$.

With $W^{ \pm}, Z^{ \pm}$given in (A.40) and $\alpha_{1}^{L, R}$ given in (A.55), we compute:

$$
\begin{aligned}
& Z^{+}+W^{+} \mathbf{j}=\frac{2 \pi}{\mu_{0}\left|\Omega_{1}\right|} g_{+}(\mathcal{Z}+\mathcal{W} \mathbf{j}) g_{-}^{\dagger}, \\
& Z^{-}+W^{-} \mathbf{j}=\frac{2 \pi}{\mu_{0}\left|\Omega_{1}\right|} g_{-}(\mathcal{Z}+\mathcal{W} \mathbf{j}) g_{+}^{\dagger},
\end{aligned}
$$

where we used the normalization $\Omega_{1}=\left|\Omega_{1}\right|$. Otherwise, the formulas (A.59) are corrected by the multiplication of $\mathcal{Z}, \mathcal{W}$ by the phase of $\Omega_{1}^{ \pm 1}$.

From this, it is straightforward to determine the effective complex structures $\mathcal{I}^{ \pm}$. The complex structure that multiplies $Z^{+}$and $W^{+}$by $i$ amounts to left multiplication of the quaternion $Z^{+}+W^{+} \mathbf{j}$ by $\mathbf{i}$. This is equivalent to left multiplication of the quaternion $\mathcal{Z}+\mathcal{W} \mathbf{j}$ by $g_{+}^{\dagger} \mathbf{i} g_{+}$. So $\mathcal{I}^{+}=g_{+}^{\dagger} \mathbf{i} g_{+}$, and by the same reasoning, $\mathcal{I}^{-}=g_{-}^{\dagger} \mathbf{i} g_{-}$:

$$
\begin{aligned}
& \mathcal{I}^{+}=g_{+}^{\dagger} \mathbf{i} g_{+} \\
& \mathcal{I}^{-}=g_{-}^{\dagger} \mathbf{i} g_{-}
\end{aligned}
$$

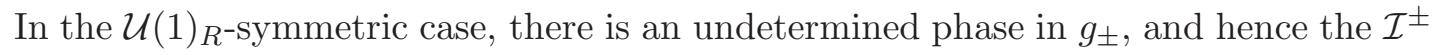
are only naturally determined up to a rotation around the $I$ axis. This is good enough for determining the effective $A$-model, since in $\mathcal{U}(1)_{R}$-invariant theories (and more generally in any $\mathcal{N}=2$ model without hypermultiplet bare masses, even if $\mathcal{U}(1)_{R}$ is anomalous), only the part of the complexified Kahler class proportional to $\omega_{I}$ is relevant. This was explained in section 2.3.2, and reflects the fact that, in such examples, the other hyper-Kahler forms $\omega_{J}$ and $\omega_{K}$ are cohomologically trivial. In a model that is not $\mathcal{U}(1)_{R}$-symmetric, $\omega_{J}$ and 
$\omega_{K}$ may be cohomologically non-trivial, in which case more detailed input about the model is needed to fully determine the effective $A$-model.

\section{A.4.1 Mapping to the $A$-Model}

Suppose that we fix a general pair $\left(\mathcal{I}^{+}, \mathcal{I}^{-}\right)$of left- and right- moving complex structures in a model of this type with $(4,4)$ supersymmetry. The general formulas for the effective parameters of the resulting $A$-model were given in eq. (2.26).

It is convenient to make these formulas more explicit as follows. First, write

$$
\mathcal{I}^{ \pm}=\zeta_{1}^{ \pm} \mathbf{i}+\zeta_{2}^{ \pm} \mathbf{j}+\zeta_{13}^{ \pm} \mathbf{k}
$$

where $\vec{\zeta}^{ \pm}=\left(\zeta_{1}^{ \pm}, \zeta_{2}^{ \pm}, \zeta_{3}^{ \pm}\right)$both have unit norm, $\vec{\zeta}^{ \pm} \cdot \vec{\zeta}^{ \pm}=1$. Then expand the symplectic form $\omega$ and the two-form field $B$ of the effective $A$-model

$$
\begin{aligned}
& \omega=\xi_{1} \omega_{I}^{*}+\xi_{2} \omega_{J}^{*}+\xi_{3} \omega_{K}^{*} \\
& B=\beta_{1} \omega_{I}^{*}+\beta_{2} \omega_{J}^{*}+\beta_{3} \omega_{K}^{*}
\end{aligned}
$$

in a basis of hyper-Kahler forms $\omega_{I, J, K}^{*}$ normalized with respect to the hyper-Kahler metric $g$ which one reads off from the Lagrangian (thus, $\omega_{I}^{*}=I g$, etc.). As in eq. (3.16), these forms differ by a factor $\operatorname{Im}\left(\Omega_{2} / \Omega_{1}\right)$ from the topologically normalized symplectic forms $\omega_{I, J, K}$ of eqs. (2.17) and (2.18). In particular,

$$
\omega_{I}^{*}=\operatorname{Im}\left(\Omega_{2} / \Omega_{1}\right) \omega_{I} .
$$

The vectors $\vec{\xi}, \vec{\beta}$ of eq. (A.62) are given by the simple formulas:

$$
\begin{aligned}
\vec{\xi} & =\frac{\vec{\zeta}^{+}-\vec{\zeta}^{-}}{1-\vec{\zeta}^{+} \cdot \vec{\zeta}^{-}} \\
\vec{\beta} & =\frac{\vec{\zeta}^{+} \times \vec{\zeta}^{-}}{1-\vec{\zeta}^{+} \cdot \vec{\zeta}^{-}},
\end{aligned}
$$

which follow from eq. (2.26). To be more exact, the $B$-field described in (A.64) is generated by the transformation to an effective $A$-model. We should not forget to add to it the $B$-field already present in (A.58).

Now let us compute what we can, in eq. (A.64):

$$
\begin{aligned}
\vec{\zeta}^{+} \cdot \vec{\zeta}^{-} & =-\operatorname{Re}\left(\mathcal{I}^{+} \mathcal{I}^{-}\right)=-\operatorname{Re}\left(g_{+}^{\dagger} \mathbf{i} g_{+} g_{-}^{\dagger} \mathbf{i} g_{-}\right) \\
\zeta_{1}^{+}-\zeta_{1}^{-} & =-\operatorname{Re}\left(\mathbf{i}\left(\mathcal{I}^{+}-\mathcal{I}^{-}\right)\right)=-\operatorname{Re}\left(g_{+} \mathbf{i} g_{+}^{\dagger} \mathbf{i}-g_{-} \mathbf{i} g_{-}^{\dagger} \mathbf{i}\right) \\
\left(\vec{\zeta}^{+} \times \vec{\zeta}^{-}\right)_{1} & =-\frac{1}{2} \operatorname{Re}\left(\mathbf{i}\left[\mathcal{I}^{+}, \mathcal{I}^{-}\right]\right)=\operatorname{Re}\left(\mathbf{i} g_{+}^{\dagger} \mathbf{i} g_{+} g_{-}^{\dagger} \mathbf{i} g_{-}\right) .
\end{aligned}
$$

Here we used the cyclic symmetry of the quaternion trace $\mathbf{x} \rightarrow \operatorname{Re} \mathbf{x}$. From the last two equations in (A.43), using the normalization $\Omega_{1}=\left|\Omega_{1}\right|$, we derive:

$$
\begin{aligned}
g_{+} g_{-}^{\dagger} & =\frac{1}{\left|\Omega_{1}\right|}\left(\omega_{1}+\epsilon_{1} \mathbf{j}\right) \\
g_{+} \mathbf{i} g_{-}^{\dagger} & =\frac{1}{\operatorname{Im} \Omega_{2}}\left(\omega_{2}+\epsilon_{2} \mathbf{j}\right)-\frac{\operatorname{Re} \Omega_{2}}{\operatorname{Im} \Omega_{2}} \frac{1}{\left|\Omega_{1}\right|}\left(\omega_{1}+\epsilon_{1} \mathbf{j}\right),
\end{aligned}
$$


while from the first equations in (A.43), we compute:

$$
\begin{gathered}
g_{+} \mathbf{i} g_{+}^{\dagger}=\frac{1}{\operatorname{Im}\left(\Omega_{2} \bar{\Omega}_{1}\right)} \times\left(\begin{array}{c}
\operatorname{Im}\left(\omega_{2} \bar{\omega}_{1}+\epsilon_{2} \bar{\epsilon}_{1}\right) \mathbf{i}+ \\
+\operatorname{Re}\left(\epsilon_{2} \omega_{1}-\epsilon_{1} \omega_{2}\right) \mathbf{j} \\
+\operatorname{Im}\left(\epsilon_{2} \omega_{1}-\epsilon_{1} \omega_{2}\right) \mathbf{k}
\end{array}\right) \\
g_{-} \mathbf{i} g_{-}^{\dagger}=\frac{1}{\operatorname{Im}\left(\Omega_{2} \bar{\Omega}_{1}\right)} \times\left(\begin{array}{c}
\operatorname{Im}\left(\omega_{2} \bar{\omega}_{1}+\epsilon_{1} \bar{\epsilon}_{2}\right) \mathbf{i}+ \\
+\operatorname{Re}\left(\epsilon_{2} \bar{\omega}_{1}-\epsilon_{1} \bar{\omega}_{2}\right) \mathbf{j} \\
+\operatorname{Im}\left(\epsilon_{2} \bar{\omega}_{1}-\epsilon_{1} \bar{\omega}_{2}\right) \mathbf{k}
\end{array}\right) .
\end{gathered}
$$

Armed with eqs. (A.66) and (A.67), we finish the computation in (A.65). We compute

$$
\begin{aligned}
\vec{\zeta}^{+} \cdot \vec{\zeta}^{-} & =\frac{\left|\omega_{1}\right|^{2}-\left|\epsilon_{1}\right|^{2}}{\left|\omega_{1}\right|^{2}+\left|\epsilon_{1}\right|^{2}} \Longrightarrow 1-\vec{\zeta}^{+} \cdot \vec{\zeta}^{-}=\frac{2\left|\epsilon_{1}\right|^{2}}{\left|\Omega_{1}\right|^{2}} \\
\zeta_{1}^{+}-\zeta_{1}^{-} & =2 \frac{\operatorname{Im}\left(\epsilon_{2} \bar{\epsilon}_{1}\right)}{\operatorname{Im}\left(\Omega_{2} \bar{\Omega}_{1}\right)} \\
\left(\vec{\zeta}^{+} \times \vec{\zeta}^{-}\right)_{1} & =\frac{1}{\operatorname{Im}\left(\Omega_{2} \bar{\Omega}_{1}\right)}\left(-\operatorname{Re}\left(\omega_{2} \bar{\omega}_{1}\right)+\operatorname{Re}\left(\epsilon_{2} \bar{\epsilon}_{1}\right)+\left(\left|\omega_{1}\right|^{2}-\left|\epsilon_{1}\right|^{2}\right) \operatorname{Re}\left(\Omega_{2} / \Omega_{1}\right)\right) \\
& =\frac{2\left|\epsilon_{1}\right|^{2}}{\operatorname{Im}\left(\Omega_{2} \bar{\Omega}_{1}\right)}\left(\operatorname{Re}\left(\epsilon_{2} / \epsilon_{1}\right)-\operatorname{Re}\left(\Omega_{2} / \Omega_{1}\right)\right) .
\end{aligned}
$$

These combine to give us:

$$
\begin{gathered}
\xi_{1}=\frac{\operatorname{Im}\left(\epsilon_{2} / \epsilon_{1}\right)}{\operatorname{Im}\left(\Omega_{2} / \Omega_{1}\right)} \\
\beta_{1}=\frac{\operatorname{Re}\left(\epsilon_{2} / \epsilon_{1}\right)-\operatorname{Re}\left(\Omega_{2} / \Omega_{1}\right)}{\operatorname{Im}\left(\Omega_{2} / \Omega_{1}\right)}
\end{gathered}
$$

and

$$
\begin{aligned}
& \omega=\operatorname{Im}\left(\epsilon_{2} / \epsilon_{1}\right) \omega_{I}+\ldots \\
& B=\operatorname{Re}\left(\epsilon_{2} / \epsilon_{1}\right) \omega_{I}+\ldots
\end{aligned}
$$

In arriving at this $B$-field, we have included the bare $B$-field from (A.58), and of course we have also taken (A.63) into account. Finally, we get the complexified Kahler class of the effective $A$-model:

$$
B+i \omega=\frac{\epsilon_{2}}{\epsilon_{1}} \omega_{I}+\ldots
$$

This formula was obtained much more simply for a special choice of the $\Omega$-deformation parameters in eq. (4.7). Obtaining it in general has been the main goal of the present appendix.

\section{A.5 Dehn twists}

In our setup, the torus $T_{\circ}$ had a preferred 1-cycle. The second 1-cycle is not defined uniquely. We could have made a Dehn twist of the torus:

$$
\left(\alpha_{1}, \alpha_{2}\right) \mapsto\left(\alpha_{1}+m \alpha_{2}, \alpha_{2}\right)
$$


for any integer $m \in \mathbb{Z}$. This is a symmetry of (A.32) provided the parameters $\left(\omega_{1}, \omega_{2}\right)$, $\left(\epsilon_{1}, \epsilon_{2}\right)$ are transformed accordingly:

$$
\left(\omega_{1}, \omega_{2}\right) \mapsto\left(\omega_{1}, \omega_{2}-m \omega_{1}\right), \quad\left(\epsilon_{1}, \epsilon_{2}\right) \mapsto\left(\epsilon_{1}, \epsilon_{2}-m \epsilon_{1}\right)
$$

The effect of this transformation on our sigma-model is clear from (A.71): the $B$-field is shifted by an integer multiple of $\omega_{I}$, which corresponds to the presence of a Chan-Paton curvature. Note also that $\tau_{\mathcal{N}=4}$ is shifted by $m$.

Open Access. This article is distributed under the terms of the Creative Commons Attribution Noncommercial License which permits any noncommercial use, distribution, and reproduction in any medium, provided the original author(s) and source are credited.

\section{References}

[1] G.W. Moore, N. Nekrasov and S. Shatashvili, Integrating over Higgs branches, Commun. Math. Phys. 209 (2000) 97 [hep-th/9712241] [SPIRES].

[2] A.A. Gerasimov and S.L. Shatashvili, Higgs bundles, gauge theories and quantum groups, Commun. Math. Phys. 277 (2008) 323 [hep-th/0609024] [SPIRES].

[3] A.A. Gerasimov and S.L. Shatashvili, Two-dimensional gauge theories and quantum integrable systems, arXiv:0711.1472 [SPIRES].

[4] N.A. Nekrasov and S.L. Shatashvili, Supersymmetric vacua and Bethe ansatz, Nucl. Phys. Proc. Suppl. 192-193 (2009) 91 [arXiv:0901.4744] [SPIRES].

[5] N.A. Nekrasov and S.L. Shatashvili, Quantum integrability and supersymmetric vacua, Prog. Theor. Phys. Suppl. 177 (2009) 105 [arXiv:0901.4748] [SPIRES].

[6] N.A. Nekrasov and S.L. Shatashvili, Quantization of integrable systems and four dimensional gauge theories, arXiv:0908.4052 [SPIRES].

[7] N.A. Nekrasov, Seiberg-Witten prepotential from instanton counting, Adv. Theor. Math. Phys. 7 (2004) 831 [hep-th/0206161] [SPIRES].

[8] A. Losev, N. Nekrasov and S.L. Shatashvili, Issues in topological gauge theory, Nucl. Phys. B 534 (1998) 549 [hep-th/9711108] [SPIRES].

[9] A. Lossev, N. Nekrasov and S.L. Shatashvili, Testing Seiberg-Witten solution, NATO Advanced Study Institute on Strings, Branes and Dualities, Cargese, France (1997) hep-th/9801061 [SPIRES].

[10] A. Kapustin and D. Orlov, Remarks on A-branes, mirror symmetry and the Fukaya category, J. Geom. Phys. 48 (2003) 84 [hep-th/0109098] [SPIRES].

[11] A. Kapustin, A-branes and noncommutative geometry, hep-th/0502212 [SPIRES].

[12] M. Aldi and E. Zaslow, Coisotropic branes, noncommutativity and the mirror correspondence, JHEP 06 (2005) 019 [hep-th/0501247] [SPIRES].

[13] A. Kapustin and E. Witten, Electric-magnetic duality and the geometric Langlands program, hep-th/0604151 [SPIRES].

[14] S. Gukov and E. Witten, Branes and quantization, arXiv:0809.0305 [SPIRES]. 
[15] L.F. Alday, D. Gaiotto and Y. Tachikawa, Liouville correlation functions from four-dimensional gauge theories, Lett. Math. Phys. 91 (2010) 167 [arXiv:0906.3219] [SPIRES].

[16] D. Gaiotto, N=2 dualities, arXiv:0904.2715 [SPIRES].

[17] N. Hitchin, The self-duality equations on a Riemann surface, Proc. Lond. Math. Soc. 55 (1987) 59 [SPIRES].

[18] N.C. Leung and C. Vafa, Branes and toric geometry, Adv. Theor. Math. Phys. 2 (1998) 91 [hep-th/9711013] [SPIRES].

[19] E. Frenkel, Affine algebras, langlands duality and Bethe Ansatz, q-alg/9506003 [SPIRES].

[20] B. Feigin, E. Frenkel and N. Reshetikhin, Gaudin model, Bethe ansatz and correlation functions at the critical level, Commun. Math. Phys. 166 (1994) 27 [hep-th/9402022] [SPIRES].

[21] E. Frenkel, Gaudin model and opers, math/0407524 [SPIRES].

[22] B. Feigin, E. Frenkel and V. Toledano Laredo, Gaudin models with irregular singularities, Adv. Math. 223 (2010) 873 [math/0612798] [SPIRES].

[23] B.Feigin and E.Frenkel, Affine Kac-Moody algebras at the critical level and Gelfand-Dikii algebras, Int. J. Mod. Phys. A Suppl. 1A (1992) 197.

[24] E. Frenkel, Lectures on the Langlands program and conformal field theory, hep-th/0512172 [SPIRES].

[25] J. Teschner, Quantum geometric langlands vs. non-perturbative dualities in $\Sigma$ models, http://online.kitp.ucsb.edu/online/duality09/teschner1/.

[26] J. Teschner, Notes on Liouville theory, quantization of the hitchin system, and the geometric Langlands correspondence, to appear.

[27] A. Losev, N. Nekrasov and S.L. Shatashvili, The freckled instantons, hep-th/9908204 [SPIRES].

[28] V. Pestun, Topological strings in generalized complex space, Adv. Theor. Math. Phys. 11 (2007) 399 [hep-th/0603145] [SPIRES].

[29] A. Connes, M.R. Douglas and A.S. Schwarz, Noncommutative geometry and matrix theory: Compactification on tori, JHEP 02 (1998) 003 [hep-th/9711162] [SPIRES].

[30] N. Seiberg and E. Witten, String theory and noncommutative geometry, JHEP 09 (1999) 032 hep-th/9908142 [SPIRES].

[31] R. Donagi and E. Witten, Supersymmetric Yang-Mills theory and integrable systems, Nucl. Phys. B 460 (1996) 299 [hep-th/9510101] [SPIRES].

[32] N. Hitchin, Flat connections and geometric quantization, Commun. Math. Phys. 131 (1990) 347] [SPIRES].

[33] A. Beilinson and V. Drinfeld, Quantization of Hitchin's integrable system and Hecke Eigensheaves, http://www.math.uchicago.edu/ mitya/langlands.html (1995).

[34] N. Seiberg and E. Witten, Gauge dynamics and compactification to three dimensions, hep-th/9607163 [SPIRES].

[35] E. Witten, Topological quantum field theory, Commun. Math. Phys. 117 (1988) 353 [SPIRES]. 
[36] J.A. Harvey, G.W. Moore and A. Strominger, Reducing S-duality to T-duality, Phys. Rev. D 52 (1995) 7161 [hep-th/9501022] [SPIRES].

[37] M. Bershadsky, A. Johansen, V. Sadov and C. Vafa, Topological reduction of 4-d SYM to 2-d $\Sigma$ models, Nucl. Phys. B 448 (1995) 166 [hep-th/9501096] [SPIRES].

[38] D. Gaiotto, G.W. Moore and A. Neitzke, Four-dimensional wall-crossing via three-dimensional field theory, arXiv:0807.4723 [SPIRES].

[39] S.J. Gates, Jr., C.M. Hull and M. Roček, Twisted multiplets and new supersymmetric nonlinear $\sigma$-models, Nucl. Phys. B 248 (1984) 157 [SPIRES].

[40] N. Hitchin, Generalized Calabi-Yau manifolds, Quart. J. Math. Oxford Ser. 54 (2003) 281 [math/0209099] [SPIRES].

[41] M. Gualtieri, Generalized complex geometry, math/0401221 [SPIRES].

[42] D. Gaiotto and E. Witten, Supersymmetric boundary conditions in $N=4$ super Yang-Mills theory, arXiv:0804.2902 [SPIRES].

[43] N. Wyllard, $A_{N-1}$ conformal Toda field theory correlation functions from conformal $N=2 \mathrm{~S} U(N)$ quiver gauge theories, JHEP 11 (2009) 002 [arXiv:0907.2189] [SPIRES].

[44] A. Mironov, S. Mironov, A. Morozov and A. Morozov, CFT exercises for the needs of AGT, arXiv:0908.2064 [SPIRES].

[45] T. Hausel and M. Thaddeus, Mirror symmetry, langlands duality and the hitchin system, Invent. Math. 153 (2003) 197.

[46] L. Chekhov and V.V. Fock, A quantum Teichmüller space, Theor. Math. Phys. 120 (1999) 1245 [Teor. Mat. Fiz. 120 (1999) 511] [math/9908165] [SPIRES].

[47] V.V. Fock and A.B. Goncharov, The quantum dilogarithm and representations of quantum cluster varieties, Invent. Math. 175 (2009) 223.

[48] D. Gaiotto and E. Witten, Supersymmetric boundary conditions in $N=4$ super Yang-Mills theory, arXiv:0804.2902 [SPIRES].

[49] J.E. Andersen, J. Mattes and N. Reshetikhin, The Poisson structure on the moduli space of flat connections and chord diagrams, Topology 35 (1996) 1069.

[50] E. Witten, Gauge theories, vertex models and quantum groups, Nucl. Phys. B 330 (1990) 285 [SPIRES].

[51] J.E. Andersen, J. Mattes and N. Reshetikhin, Quantization of the algebra of chord diagrams, Math. Proc. Camb. Phil. Soc. 124 (1998) 451.

[52] E. Witten, Quantum field theory and the Jones polynomial, Commun. Math. Phys. 121 (1989) 351 [SPIRES].

[53] J.E. Andersen, Hitchin's connection, Toeplitz operators and symmetry-invariant deformation quantization, math/0611126.

[54] E. Verlinde, Fusion rules and modular transformations in 2D conformal field theory, Nucl. Phys. B 300 (1988) 360 [SPIRES].

[55] L.F. Alday, D. Gaiotto, S. Gukov, Y. Tachikawa and H. Verlinde, Loop and surface operators in $\mathcal{N}=2$ gauge theory and Liouville modular geometry, arXiv:0909.0945 [SPIRES].

[56] N. Drukker, J. Gomis, T. Okuda and J. Teschner, Gauge theory loop operators and Liouville theory, arXiv:0909.1105 [SPIRES]. 
[57] N. Drukker, D.R. Morrison and T. Okuda, Loop operators and S-duality from curves on Riemann surfaces, JHEP 09 (2009) 031 [arXiv:0907.2593] [SPIRES].

[58] V. Pestun, Localization of gauge theory on a Four-Sphere and supersymmetric Wilson loops, arXiv:0712.2824 [SPIRES].

[59] S. Cecotti and C. Vafa, Topological antitopological fusion, in Proceedings of High Energy Physics and Cosmology, vol. 2, Trieste (1991) pg. 682.

[60] D. Friedan and S.H. Shenker, The analytic geometry of two-dimensional conformal field theory, Nucl. Phys. B 281 (1987) 509 [SPIRES].

[61] L.F. Alday, D. Gaiotto, S. Gukov, Y. Tachikawa and H. Verlinde, Loop and surface operators in $\mathcal{N}=2$ gauge theory and Liouville modular geometry, arXiv:0909.0945 [SPIRES].

[62] D. Gaiotto, Surface operators in $\mathcal{N}=24 d$ gauge theories, arXiv:0911.1316 [SPIRES]. 